CERN-PPE/91-234

$16^{\text {th }}$ December 1991.

\title{
The Detector Simulation Program for the OPAL Experiment at LEP
}

J. Allison ${ }^{16}$, J. Banks ${ }^{16}$, R.J. Barlow ${ }^{16}$, J.R. Batley ${ }^{6}$, O. Biebel ${ }^{4}$, R. Brun ${ }^{9}$, A. Buijs ${ }^{9}$, F.W. Bullock ${ }^{15}$, C.Y. Chang ${ }^{17}$, J.E. Conboy ${ }^{15}$, R. Cranfield ${ }^{15}$, G.M. Dallavalle ${ }^{2}$, M. Dittmar ${ }^{5}$, J.-J. Dumont ${ }^{7, a}$, C. Fukunaga ${ }^{21, f}$, J.W. Gary ${ }^{5}$, J. Gascon ${ }^{18}$, N.I. Geddes ${ }^{19}$, S.W. Gensler ${ }^{10}$, V. Gibson ${ }^{6}$, J.D. Gillies ${ }^{19}$, J. Hagemann ${ }^{12}$, M. Hansroul ${ }^{9}$, P.F. Harrison ${ }^{14}$, J. Hart ${ }^{6}$, P.M. Hattersley ${ }^{1}$, M. Hauschild ${ }^{9}$, R.J. Hemingway ${ }^{7}$, F.F. Heymann ${ }^{15}$, P.R. Hobson ${ }^{22}$, D. Hochman ${ }^{23}$, R. Hospes ${ }^{4}$, R.W.L. Jones ${ }^{14}$, K. Kawagoe ${ }^{21}$, T. Kawamoto ${ }^{21}$, B.W. Kennedy ${ }^{15}$, L. Köpke ${ }^{9}$, R. Kowalewski ${ }^{7}$, H. Kreutzmann ${ }^{4}$, G.D. Lafferty ${ }^{16}$, J.G. Layter ${ }^{5}$, D. Lellouch ${ }^{23}$, S.L. Lloyd $^{14}$, B. Lorazo $^{18}$, M.J. Losty ${ }^{8}$, M.L. Luvisetto ${ }^{3}$, A.C. McPherson ${ }^{7, b}$, T. Mashimo ${ }^{21}$, P. Mättig ${ }^{4}$, J. Mildenberger ${ }^{7}$, W.J. Murray ${ }^{6, c}$, S.W. O'Neale ${ }^{9, d}$, M.J. Oreglia ${ }^{10}$, G.N. Patrick ${ }^{19}$, S.J.Pawley ${ }^{16}$, P.Pfister ${ }^{11}$, A.Possoz ${ }^{10, a}$, E.Prebys ${ }^{9}$, G. Quast ${ }^{12}$, M.W. Redmond ${ }^{10}$, D.L. Rees ${ }^{1}$, K. Riles ${ }^{5}$, C.M. Roach ${ }^{5, e}$, A. Rossi ${ }^{2}$, P. Routenburg ${ }^{7}$, A.D. Schaile ${ }^{11}$, G. Tysarczyk-Niemeyer ${ }^{13}$,

G.J. VanDalen ${ }^{5}$, R. Van Kooten ${ }^{9}$, C.P. Ward ${ }^{6}$, D.R. Ward ${ }^{6}$, P.M. Watkins ${ }^{1}$, N.K. Watson ${ }^{9}$, S. Weisz ${ }^{9}$, G.W.Wilson ${ }^{20}$, R. Yaari ${ }^{23}$, P. Zanarini ${ }^{2}$.

\begin{abstract}
The Monte Carlo program for the OPAL experiment at the LEP $e^{+} e^{-}$collider is described. This program is based on the GEANT simulation package. The general organization of the program is outlined, and a description is given of the techniques used for simulating the various subdetectors of OPAL. The performance of the program is illustrated by comparisons with recent data recorded by OPAL at LEP.
\end{abstract}

(Submitted to Nucl. Instr. and Meth.) 
${ }^{1}$ School of Physics and Space Research, University of Birmingham, Birmingham, B15 2TT, UK ${ }^{2}$ Dipartimento di Fisica dell' Università di Bologna and INFN, Bologna, 40126, Italy

${ }^{3}$ CNAF-INFN, Bologna, Italy

${ }^{4}$ Physikalisches Institut, Universität Bonn, D-5300 Bonn 1, FRG

${ }^{5}$ Department of Physics, University of California, Riverside, CA 92521 USA

${ }^{6}$ Cavendish Laboratory, Cambridge, CB3 0HE, UK

${ }^{7}$ Carleton University, Dept of Physics, Colonel By Drive, Ottawa, Ontario K1S 5B6, Canada

${ }^{8}$ Centre for Research in Particle Physics, Carleton University, Ottawa, Ontario K1S 5B6,

Canada

${ }^{9}$ CERN, European Organisation for Particle Physics, 1211 Geneva 23, Switzerland

${ }^{10}$ Enrico Fermi Institute and Department of Physics, University of Chicago, Chicago Illinois 60637, USA

${ }^{11}$ Fakultät für Physik, Albert Ludwigs Universität, D-7800 Freiburg, FRG

${ }^{12}$ Universität Hamburg/DESY, II Inst. für Experimental Physik, 2000 Hamburg 52, FRG

${ }^{13}$ Physikalisches Institut, Universität Heidelberg, Heidelberg, FRG

${ }^{14}$ Queen Mary and Westfield College, University of London, London, E1 4NS, UK

${ }^{15}$ University College London, London, WC1E 6BT, UK

${ }^{16}$ Department of Physics, Schuster Laboratory, The University, Manchester, M13 9PL, UK

${ }^{17}$ Department of Physics and Astronomy, University of Maryland, College Park, Maryland 20742, USA

${ }^{18}$ Laboratoire de Physique Nucléaire, Université de Montréal, Montréal, Quebec, H3C 3J7, Canada

${ }^{19}$ Rutherford Appleton Laboratory, Chilton, Didcot, Oxfordshire, OX11 0QX, UK

${ }^{20}$ DPhPE, CEN Saclay, F-91191 Gif-sur-Yvette, France

${ }^{21}$ International Centre for Elementary Particle Physics, University of Tokyo, Tokyo 113, Japan

${ }^{22}$ Brunel University, Uxbridge, Middlesex, UB8 3PH UK

${ }^{23}$ Nuclear Physics Department, Weizmann Institute of Science, Rehovot, 76100, Israel

${ }^{a}$ Present address: EPFL, Lausanne, Switzerland

${ }^{b}$ Present address: Applied Silicon Inc, Ottawa, Canada

${ }^{c}$ Present address: Rutherford Appleton Laboratory, Chilton, Didcot, Oxfordshire, OX11 0QX, $\mathrm{UK}$

${ }^{d}$ On leave from Birmingham University, Birmingham B15 2TT, UK

${ }^{e}$ Present address: Culham Laboratory, Culham, Oxfordshire, UK

${ }^{f}$ Present address: Meiji Gakuin University, Yokohama 244, Japan 


\section{Introduction}

Monte Carlo programs for detector simulation are an essential tool in understanding and analysing data from large experiments in High Energy Physics. They are also important in assisting the design of the detector and in allowing the data analysis code to be developed in advance of data-taking. The OPAL experiment [1] at the large $e^{+} e^{-}$collider LEP at CERN uses a Monte Carlo program called GOPAL. This program is based on the CERN GEANT simulation package [2], which provides a framework for the definition of the detector geometry, and controls the tracking of particles through this geometry using well-tested routines to simulate interactions.

The general organization of the OPAL simulation system is indicated by Fig. 1. It is possible to run the whole simulation and analysis chain shown in Fig. 1 in a single job, or in separate stages. For example, the physics event generator may be run inside GOPAL, or may be run separately and the results written to a file which can be read by GOPAL. Likewise, the constants required by GOPAL to define the detector configuration may be taken from defaults embedded in the program, or read from the OPAL database. The output from GOPAL is a copy of the constants used, together with simulated "raw data" (in the same format as provided by the real detector), which can be analysed by the OPAL event reconstruction program ROPE to produce a data summary tape (DST) for analysis. However, the user has the option to run the ROPE reconstruction code within GOPAL, and write out a DST, or even include analysis code inside GOPAL and just write out histograms or "N-tuples". The program also incorporates a fast mode of running inside the GOPAL framework, which fills the DST structure without detailed simulation of raw data and reconstruction.

In section 2 we discuss various aspects of the organization of the GOPAL program, and describe some of the features available to the user. Then in section 3 we describe the procedures used for simulating the response of each of the OPAL subdetectors, and evaluate their performance by comparing the results of GOPAL with corresponding OPAL data taken at LEP. In section 4 we describe the fast "smear" mode of the program.

\section{The OPAL Monte Carlo Program - GOPAL}

\subsection{The OPAL Detector}

A detailed description of the OPAL detector has been given in Ref. [1], and some salient features will be mentioned in describing the detector simulation in section 3 below. OPAL was conceived as a general purpose detector for recording the decays of the $\mathrm{Z}^{0}$ with an acceptance of almost $4 \pi$ in solid angle. It permits the detection of charged particles, photons and neutral hadrons, with the identification of electrons and muons in complex jet environments. In general the design of OPAL was based on relatively conventional technology.

Note that the OPAL coordinate system referred to throughout the paper is defined so that $z$ is the coordinate parallel to the beam axis, with $+z$ along the direction of the $e^{-}$beam; $r$ is the coordinate normal to the beam axis; $\phi$ is the azimuthal angle about $z$, and $\theta$ is the polar angle with respect to $z$.

In brief, the main features of the OPAL detector are: 
- A silicon microvertex detector (installed in 1991) surrounding a beryllium beam pipe. This detector consists of two layers of microstrip detectors providing high resolution coordinates in the transverse plane.

- A central detector, which consists of three systems of drift chambers within a large volume pressure vessel - an inner vertex detector, a large jet chamber for momentum and $d E / d x$ determination, surrounded by Z-chambers to give more precise measurements in the longitudinal direction.

- A warm solenoidal coil, providing a uniform field within the central detector of about $0.43 \mathrm{~T}$ along the beam axis.

- Time-of-flight scintillation counters arranged in a barrel around the coil.

- An electromagnetic calorimeter, which consists of 9440 lead glass blocks in the barrel, and 2264 lead glass blocks in the endcap. Presamplers immediately before the lead glass permit corrections for energy loss in the coil and pressure vessel, and localization of shower positions.

- The magnet return iron, which is instrumented as a hadronic calorimeter.

- Muon chambers (drift chambers and limited streamer tubes) surrounding the entire detector.

- A forward detector, including a lead-scintillator calorimeter, used principally for luminosity determination.

\subsection{General Structure of GOPAL}

When the GOPAL program starts executing it first passes through an initialization phase, in which the geometrical configuration of the detector is specified in the form required by GEANT. The program then loops over a given number of events to be generated. First the kinematics of the particles forming the primary event are obtained. Each of these particles in turn is tracked by the GEANT routines, and when a particle traverses a sensitive region of the detector suitable information (called "HITS" in GEANT terminology) is stored. At the end of tracking the HITS are collected for each detector in turn and the response of the detector is computed, generating simulated raw data (called "DIGITS" in GEANT terminology). Optionally the reconstruction of these data may also occur within GOPAL. These stages will be described in the following sections.

\subsection{Initialization and Constants}

The initialization phase of the program is mainly concerned with defining the geometry of the detector, and the materials and tracking media to be used by GEANT. In addition many of the constants to be used in the digitization phase are set up. OPAL comprises 15 subdetectors, together with some passive elements (such as the magnet coil, beam pipe, pressure vessel). The user can choose to simulate any combination or all of these using data cards. 
The geometrical description of OPAL using GEANT has been described in Ref. [3]. Figures 2 and 3 show two views of the OPAL detector as implemented in GOPAL. The full geometrical representation of OPAL in GOPAL requires the definition of 49 materials and 68413 volumes, nested up to 12 deep in some cases.

In the early versions of the OPAL Monte Carlo program all the constants required were simply hard-coded in the program. The OPAL database only became available at about the time when the first LEP data were taken. Since then we have evolved alternative ways of defining constants, which can be chosen independently for each subdetector:

either The constants data structure can be read from the database, for a specified run number. This is the same data structure (or possibly a subset of it) that would be read by the reconstruction program for real data, and the data contained in it are used in setting up the geometry, and in digitization.

or The same data structure (or the necessary parts of it) is set up in a defaults routine in GOPAL using hard-wired constants, possibly modified using data cards.

A standard run of the program is one in which the database is not used, and in which no data cards are used to override defaults. This means that the defaults in the program are set to realistic, not idealized, values. Generally speaking the database option is mainly used by experts for specialist studies.

Whichever method is chosen for defining the constants, the constants data structure is written to the event output stream, just before the first event. This constants structure is then read and recognised by the reconstruction program ROPE, and set up just as if it had been read from the database. The reconstruction code can then operate in just the same way as for real data, but without using the database directly. An advantage of this arrangement is that the Monte Carlo events are always reconstructed with the same set of constants that were used in the generation, and it is not necessary to keep the database maintained on all computers in the collaboration just in order to run and analyse Monte Carlo events. ${ }^{1}$

The constants banks which are used directly by GEANT (VOLU, TMED, MATE etc.), are created during the initialization phase, and these can also be written out to a separate (sequential) file. These can be read back in a subsequent GOPAL run to speed up initialization. They are also needed by the reconstruction program, where a simplified version of the GEANT tracking code is used to perform track extrapolation.

\subsection{Kinematics}

Two methods are in use for obtaining the kinematics of the particles for GEANT to track:

- The user may run the generator inside GOPAL. A user routine KIUSER has to be provided to interface the generator to GOPAL. In addition, some standard generators, particularly single-particle generators, useful for test purposes, are provided within GOPAL.

\footnotetext{
${ }^{1}$ The facility for including constants in the event stream can also be useful for real data, as a way of exporting events together with the correct constants, e.g. for event scanning on a workstation which does not have access to the full database.
} 
- The user may run the event generator in a separate stand-alone job, and write out a standard "four-vector" 2 file. This file is in a simple card-image format, and a routine within GOPAL can read and interpret such a file. This method is particularly convenient for production work, where the event generator can be run centrally at CERN, and fourvectors exported along with the GOPAL module to outside institutes. In this way there is no danger of duplicate events being generated and repetition of the same random number sequences can be avoided.

For standard generators, such as JETSET [4] and HERWIG [5], we provide model routines to run them in either of these modes.

We have found the GEANT KINE/VERT banks inadequate to contain all the kinematical information required for Monte Carlo events, such as the parton shower history, or information on decays before the GEANT tracking. We therefore use our own data structure. A TREE bank is lifted, containing 22 words per particle. The information stored is:

- four-momentum

- mass, charge and PDG particle identification code [6]

- spatial coordinates of the start and end points of the particle trajectory

- pointers to parent and daughters

- start and end flags indicating the mechanisms which created and destroyed the particle

- cross-references to GEANT KINE numbers, DST track numbers, jets etc.

In the case of the partons in a parton shower only a subset of this information is stored. Although this TREE structure contains some redundancy it is mainly designed for convenience of use in physics analysis.

The user's task in interfacing a generator to GOPAL is to fill the TREE bank. Alternatively GOPAL can create the TREE from an external four-vector file. For particles whose decay was simulated by the event generator (e.g. hadrons containing c- or b-quarks, or $\tau^{ \pm}$) it is possible to specify non-zero lifetimes in GOPAL. The particles still undecayed at the end of the TREE are then automatically copied to the GEANT KINE/VERT banks in preparation for tracking.

When secondary particles are created through GEANT interactions, they are all placed on the GEANT STACK, and some of them are also appended to the TREE bank. This may depend on the user's requirements, but typically any particles formed before or within the sensitive central detector volume, and muons created anywhere, are retained. The TREE bank is attached to the main event data structure, and is passed through to the DST, where utility routines allow the user to access the information in essentially the same way as the reconstructed event data.

\footnotetext{
${ }^{2}$ Of course this file needs to contain a little more information than just four-vectors, such as particle identification codes and history information.
} 


\subsection{Tracking and Hits}

The tracking of particles through the OPAL detector is essentially standard. In one case (the lead glass endcaps) it is convenient to use the user search facility to speed up tracking, because the geometry is such that it is easy to compute which cell a particle is in, and none of the normal GEANT optimization tools is suitable. The time taken for tracking is dominated by the need to develop electromagnetic showers in the lead glass, in which tracking must be performed down to the threshold for emission of Čerenkov light (about $100 \mathrm{keV}$ ). In most of the remainder of the detector standard tracking cutoffs (1 MeV kinetic evergy for $e^{ \pm} / \gamma$ and $10 \mathrm{MeV}$ for hadrons) are used, though in some of the sensitive volumes in the calorimetry and the muon chambers and in the thin silicon detectors lower cutoffs are employed. The automatic calculation of tracking parameters which is offered in GEANT3.14 has so far been found satisfactory.

The GEANT HITS structures are used to store information at tracking time. Typically we store the position and momentum where each charged particle traverses a sensitive volume (e.g. a drift cell in the central detector), possibly transformed to a convenient local coordinate system. Path length and energy loss are also needed in some cases. However, a more sophisticated treatment is needed for the lead glass simulation. In these detectors the whole volume is sensitive, and it is impractical to store all track segments in the shower. Therefore the detector response to each track segment is computed at tracking time using a parametrization technique (see sections 3.8 and 3.10 below), and the actual number of detected photoelectrons is stored in the HITS structure.

\subsection{Digitization}

At the end of the GEANT tracking the program enters the digitization phase, which involves collecting the HITS information and simulating the detector response. In the case of the lead glass electromagnetic calorimeter most of the detector simulation has been carried out at the HITS stage, and all that remains is to sum the signals in each block, and apply noise and a suitable normalization. For the other subdetectors a more elaborate simulation of the detector response is generally needed, as described in more detail in section 3. The GEANT DIGI data structures are not used to store the end results of the GOPAL simulation. Instead, the ZEBRA [7] banks which constitute the OPAL event data structure are created. The digitization code for each subdetector lifts the banks which would have been provided by the data acquisition system, and fills these with simulated raw data.

Thus, the data structure at the end of GOPAL should look just like that emerging from the OPAL detector. There are, of course, certain additional data specific to Monte Carlo events, such as the TREE bank, and "cheat information" (e.g. true association between particles and hits), which are important in program development and physics analysis. These data all hang from a single link in the data structure, and are thus kept separate from the raw data.

The final stage of the digitization procedure is the simulation of the OPAL trigger. The principle is to start from the raw data banks, and to simulate the trigger signals from each detector [8]. The central trigger logic which correlates these signals is then simulated. This trigger code can also be run later as a piece of analysis code (e.g. to see the effect of different trigger configurations on the trigger efficiency for some process), or can be run on real data as a check. 


\subsection{Reconstruction}

The reconstruction of Monte Carlo events is carried out by the OPAL reconstruction program ROPE (Reconstruction of OPAL Events). The output from GOPAL consists of a constants record (like that read from the database) followed by events. ROPE reads the constants record, and sets it up as if it had been read from the database, and will then read and reconstruct the simulated events just as if they were real data.

It is also possible to run the ROPE code inside GOPAL. After the digitization phase for an event GOPAL calls the appropriate steering routine from ROPE which then calls all the reconstruction processors. The event data structure and the requisite constants are already present in memory ${ }^{3}$, and the user can choose to write out a reconstructed DST (very useful on systems with limited disk or tape facilities), or data+DST, or even to include his analysis code in GOPAL and write out analysis results only.

This close integration between GOPAL and ROPE has proved very valuable. Before LEP started up GOPAL was an indispensible tool in developing the ROPE code, and the programs were usually run separately. Now that the Monte Carlo is primarily used to aid physics analysis it is often more useful to amalgamate the simulation and reconstruction stages.

\subsection{Multi-cpu Mode.}

Many of the university groups in OPAL use distributed computing systems, particularly VAX workstation clusters. In order to allow them to exploit the integrated cpu power of such a cluster in an effective way for Monte Carlo work we have implemented an elementary parallel processing option in GOPAL (for VAX/VMS only at present). In outline it works as follows:

- The same job is submitted to several nodes of the cluster.

- The jobs all run a complete version of the program, and are autonomous. They communicate through a "run file", which contains the run/event/sequence numbers for the next event to be generated, and its random number seeds. When a job is ready to start a new event it opens the run file, reads the next event number and random number seeds, sets up the kinematics, updates the run file and closes it before tracking the event. If two jobs contend for use of the run file one of them has to wait a few seconds.

- The jobs may (optionally) take their kinematical input from a shared four-vector file.

- The jobs all append to a common output file. So when a job reaches the end of an event it tries to open the output file with ACCESS='APPEND'. If successful it writes the event and closes the file. If a second job has the output file open, the first job waits a few seconds and retries.

\subsection{Graphics and Interactive Use}

The graphics facilities of GEANT were invaluable in the early stages of developing the geometry code for GOPAL. Figures 2 and 3 show typical output. However, these graphics facilities are

\footnotetext{
${ }^{3}$ This requires, of course, that ROPE use the COMMON/GCBANK/ block from GEANT as the store for holding events.
} 
now seldom used except to check when changes are made to the geometrical representation, or to debug tracking anomalies. The normal way to display Monte Carlo events is now to use the same event scanning program as is used for displaying real data and their reconstructions.

The GEANT/GOPAL graphics display is now generally used in batch mode - the interactive mode of GOPAL is only infrequently used now that the program is mature.

\subsection{Speed of GOPAL}

The full detector simulation of GOPAL is rather time consuming. This used to be a cause of concern, but recently cpu power has become so much cheaper that we have been able to produce adequate numbers of fully simulated events. For example, during 1990 more than 300000 multihadronic events for general use were generated using the computer centres at CERN, RAL, Saclay and Montreal, and many other samples have been generated for more specialized use.

Typical times for the generation of a multihadronic $\left(Z^{0} \rightarrow q \bar{q} \rightarrow\right.$ hadrons $)$ event are given in Table 1. The tracking time is dominated by shower development in the lead glass calorimeter - about two-thirds of the tracking time is spent in the lead glass, compared to about $10 \%$ in the central tracking chambers. However about half of the digitization time is spent in the tracking chambers. It should also be noted that the reconstruction time is a far from negligible component (this is again dominated by the central tracking) - so that although one would like to speed up the simulation there would be little point in speeding it up by more than a factor 4-5 unless one also bypassed or sped up the reconstruction.

In order to speed up the lead glass shower simulation we have implemented a "bootstrap" option, in which any electron or photon in the lead glass with energy below some cutoff (e.g. $500 \mathrm{MeV}$ ) is replaced by a previously generated shower chosen at random from a library. By this technique a factor of about three in speed in multihadronic events has been gained, but the method has fallen into disuse for several reasons: the bootstrap results never fitted the data quite so well as the full shower development; the parametrizations associated with the use of the showers have to be updated for each new version of GEANT; problems with leakage (especially in the electromagnetic component of hadronic showers) were never adequately resolved; and as more powerful cpus became available the I/O demands of the bootstrap method became excessive. A similar bootstrap technique is also available as an option for the forward calorimeter.

Some changes were also made to the tracking routines to speed up simulation of electromagnetic showers in the forward calorimeter, firstly by allowing up to four bremsstrahlung photons to be generated during one tracking step, and secondly by exploiting the regular structure of the forward calorimeter to predict the conversion point of photons, and skip any intermediate tracking steps. The combined effect of these modifications was a reduction of about $50 \%$ in cpu time used during tracking of Bhabha events in the forward detector.

\section{Simulation of Detector Response}

In this section we outline the simulation procedures for each subdetector of OPAL in turn. These descriptions will generally emphasize the digitization phase since an outline of the geometrical description of the detector in GOPAL has already been presented [3]. We also present some 
comparisons between the GOPAL simulation and data from OPAL operation at LEP. Unless otherwise stated the physics event generators underlying the GOPAL simulations are JETSET version 7.2 [4] for multihadronic events, with parameters tuned to OPAL data on global event shapes [9], KORALZ [10] for $\mu^{+} \mu^{-}$and $\tau^{+} \tau^{-}$events, and BABAMC [11] for Bhabha $\left(e^{+} e^{-}\right)$ events.

\subsection{Silicon Microvertex Detector (SI)}

\section{The detector geometry}

The OPAL silicon microvertex detector [12] measures the coordinates of minimum ionizing particles with a high spatial precision of $\sim 5 \mu \mathrm{m}$ at a radius close to the interaction region. It thereby provides improved identification and reconstruction of short-lived particles such as $B$ and D hadrons.

During the LEP shutdown of 1990-91, new beam-pipe configurations were installed in each of the LEP experiments. In OPAL, a new $1.1 \mathrm{~mm}$ beryllium beam-pipe with an inner radius of $5.3 \mathrm{~cm}$ was installed to minimize the amount of material between the interaction point and the silicon detectors. In addition, a replacement for the previous beam-pipe, constructed from $2.2 \mathrm{~mm}$ carbon fibre, lined with $0.05 \mathrm{~mm}$ aluminium and with an inner radius of $8.02 \mathrm{~cm}$ was used to close off the central detector pressure vessel at its inner radius. The silicon microvertex detector was inserted between the inner and outer beam-pipes.

The silicon microvertex detector consists of an array of 25 ladders arranged in 2 cylindrical layers. The inner layer has 11 ladders at a radius of $6.085 \mathrm{~cm}$ with an angular coverage of $|\cos \theta|<0.83$. The outer layer has 14 ladders at a radius of $7.515 \mathrm{~cm}$ with an angular coverage of $|\cos \theta|<0.77$. Each ladder consists of 3 silicon microstrip detectors daisy-chained together and connected to five readout chips and subsequent electronics.

The detectors are single-sided, $3.3 \times 6.0 \mathrm{~cm}$, capacitively coupled devices with $25 \mu \mathrm{m}$ strip pitch fabricated on $300 \mu \mathrm{m}$ thick high resistivity silicon [13]. The strips are orientated along the beam axis and have a $50 \mu \mathrm{m}$ readout pitch in order to measure co-ordinates in the transverse plane with an intrinsic resolution of about $5 \mu \mathrm{m}$. There are 629 readout and 630 non-readout physical strips per detector of length $\sim 5.8 \mathrm{~cm}$. In total there are 16,000 readout channels. The spacing between the detectors in $z$ is $0.025 \mathrm{~cm}$. Approximately $90 \%$ of the solid angle covered by the silicon microvertex detector is active.

The 25 ladders are supported by aluminium rings with the readout electronics situated at the negative $z$ end of the assembly. Within GOPAL, standard GEANT volumes are used to provide a full representation of the silicon detector and of the inert material, namely the kevlar ladders, aluminium rings, readout electronics, cooling pipes, an intermediate electronics interconnect ring and cables. The double beam-pipe configuration is simulated automatically if the silicon simulation is requested.

In practice, it is possible that any element of the silicon microvertex assembly may be translated or rotated with respect to its nominal position. To allow for this, a set of alignment constants have been defined and can be applied to the nominal values for each element. The basic set of alignment constants consist of 3 translations $(\Delta x, \Delta y, \Delta z)$ and 3 rotations $\left(\theta_{x}, \theta_{y}, \theta_{z}\right)$ about the $(x, y, z)$ axes of the element's local coordinate system. Within the GOPAL simulation of the silicon detector, the silicon detectors and their surrounding passive material are contained 
within the same mother volume. The mother volume is then rotated and translated with a set of alignment constants determined from the data. Since the silicon detector has a high spatial resolution, it is essential to ensure that any simulation code can reproduce positions with an equally high precision.

\section{Tracking and digitization}

GEANT extrapolates a track through the silicon detector with a precision of $1 \mu \mathrm{m}$. In traversing $300 \mu \mathrm{m}$ of silicon, the most probable energy that a minimum ionizing particle deposits is $84 \mathrm{keV}$ (1 MIP) and has a typical Landau distribution. The minimum energy cutoff for electrons and photons is set to $10 \mathrm{keV}$ for the silicon in order to produce low energy $\delta$-rays and $\gamma$ conversions.

The digitization stage converts silicon hits into strip numbers and pulse heights. The charge that would be collected is assumed to be proportional to the pathlength traversed by a track as it passes through the silicon detector. An internal field, $E$, over the $300 \mu \mathrm{m}$ of the silicon detector transports the charge carriers, in this case holes, to the surface of the silicon. The radius of the charge cloud, $\sigma$, at the surface is typically $10 \mu \mathrm{m}$ and is determined from the mean distance that the charge has to travel, $d x$, the diffusion coefficient, $\mathrm{D}$, and the mobility, $\mu$, of the charge carriers via the relationship $\sigma=\sqrt{\frac{2 D d x}{\mu E}}$ where $D=12.3 \mathrm{~cm}^{2} \mathrm{~s}^{-1}$ and $\mu=480 \mathrm{~cm}^{2}(\mathrm{Vs})^{-1}$. The diffuse charge is then collected on the strips within $\pm 3 \sigma$ from the centre of the charge cloud.

In order to simulate the division of charge between the capacitively coupled non-readout and readout strips, it is assumed that the intermediate strips lose $50 \%$ of their charge to each of their neighbouring readout strips, thus having the effect of making the charge division more linear.

In multihadronic data, the silicon clusters are found to have a signal-to-noise of $\sim 20: 1$. Random noisy channels are therefore not simulated in the silicon since they can be removed with high efficiency in data using a pulse height cut. However, random noise is added to the pulse heights produced by real hits and is taken from a Gaussian distribution with a width of 0.05 MIP. The single hit efficiency of a ladder has been determined from the data to be $97 \%$. During the 1991 run, 2 ladders were not used for data analysis and hence were inactivated in the Monte Carlo. The digitized Monte Carlo output is stored in a data structure identical structure to that of the data. Pulse heights are stored in units of MIPs.

\section{Comparison with data}

A simple cluster finding algorithm is employed, where a cluster is defined as any channel whose pulse height is $>4.0 \sigma$ (where $\sigma$ is the r.m.s. noise) and includes the \pm 2 neighbouring channels if they have pulse heights $>1.5 \sigma$ and are contiguous.

A comparison of the silicon simulation with data from multihadron events is shown in Fig. 4 . Figure 4(a) shows the total number of clusters in the inner silicon barrel, Fig. 4(b) compares the pulse height distributions, Fig. 4(c) shows the number of tracks to which two silicon hits could be assigned, and Fig. 4(d) shows the distance of closest approach to the event vertex for such tracks. In general the agreement is good. The small discrepancies at low and high pulse heights in the Landau distribution arise because the online processing algorithm and anomalously high pulse heights are not yet simulated in GOPAL. 


\subsection{Vertex Detector (CV).}

\section{The detector geometry}

The OPAL vertex chamber [14] provides high precision measurements of charged tracks in the $r-\phi$ plane, a fast measurement of the $z$-coordinate for triggering purposes and a more precise $z$-measurement after track-matching with the jet chamber. The chamber is composed of two separated cylinders - an inner volume with axial wires, and an outer volume with stereo wires. The inner volume consists of 36 axial jet cells, each with a radial plane of 12 anode sense wires at radii from $10.3 \mathrm{~cm}$ to $16.2 \mathrm{~cm}$. The anode wires are staggered by $40 \mu \mathrm{m}$ to either side of the anode plane. The outer layer volume contains 36 stereo jet cells, each with a plane of 6 wires at radii from 19.1 to $21.6 \mathrm{~cm}$ and a twisted geometry, with the two end-plates rotated in opposite senses by $10^{\circ}$. Stereo hits may be associated with an external track in $r-\phi$; the precise knowledge of the $r-\phi$ position of the stereo hits from the external information then allows their $z$-coordinate to be determined accurately.

The geometry of the detector, its support and preamplification electronics are modelled using standard GEANT volumes. All volumes are described by the appropriate materials or mixtures of materials.

\section{Tracking and digitization}

Charged tracks are followed through the chamber using the standard GEANT tracking. The drift volume for each cell is defined by two planes, at half the wire spacing either side of a wire, tilted by the Lorentz angle with respect to the perpendicular to the anode plane. The points at which a track crosses these planes are stored (as GEANT "HITS"), though these hits may not necessarily survive the digitization simulation.

In the real detector the time of arrival of the ionization from a track on each wire is measured using TDC modules. The wire signals are read out into electronics that have a multi-hit capability, so that several hits may be registered on the same wire in a given event. The difference in arrival time of the pulse at either end of the sense wire is also recorded, and this is a measure of the $z$-coordinate of the hit (the 'fast- $z$ ') which is used in triggering.

The effects of resolution and inefficiencies of the detector are modelled in the digitization stage. The effective drift distance for each hit to the anode wire is calculated as the average of the distances to the anode plane for the points of entry and exit for each drift cell, combined with the components of the electrostatic bowing and the wire stagger in the drift direction. The spatial resolution of the detector in the $r-\phi$ plane depends on the drift distance for the hit and has been parameterised by a function fitted to real data. Those hits which have a preceding hit recorded on the same wire ('second' hits) have a resolution that is somewhat worse than those that do not ('first' hits). In the simulation, the resolution for second hits takes the same form in drift distance, but is degraded by an additional factor. Once the resolution for a given hit has been calculated, the drift distance is smeared accordingly.

For all classes of hits, a small fraction fail to be recorded due to inefficiencies. These inefficiencies are measured from the data, and a proportion of the hits are discarded on a pseudo-random basis. The efficiency for registering second hits is lower than that for registering first hits. There is a region of zero efficiency after the first hit due to the dead time of the electronics, after which there is a slow turn-on, which rises to a plateau somewhat lower than the first hit efficiency. The rate of this turn-on depends on the number of tracks that passed between the current hit and the previous hit. All of these effects are modelled, using parametrisations taken 
from the data.

The effective drift distance is then converted to a TDC value to simulate the raw data from the real detector. The form of the distance-to-time relationship has been determined using test chambers and simulations of the ionization and drift processes and the electrostatics of the final chamber, and the parameters have been tuned to correspond to the data from the real chamber. The time thus obtained is converted to a TDC value using the known clock frequency and an additional time offset contribution is added at this stage to model the time delay between the starting of the TDC clocks and the arrival of the earliest pulses.

The 'fast- $z$ ' measurement is also simulated. The true $z$ of the hit is taken from the average $z$ of the entry and exit points for each drift cell and this value is smeared by a resolution determined from data.

In the real detector there is a noise background, unassociated with particles from $e^{+} e^{-}$interactions, which is largely due to synchrotron radiation. The level of this background per wire is obtained from looking at random beam crossings in the real data. Hits are then generated according to this distribution at the beginning of the digitization step, and can contribute to the 'second hit' effects described above.

\section{Comparison with data}

The performance of the CV simulation is largely measured by the quality of agreement between the impact parameter distributions in data and Monte Carlo for tracks including CV hits, and by the distribution of the number of $\mathrm{CV}$ hits on tracks. Both of these distributions will be shown and discussed below (section 3.5).

The good agreement between data and Monte Carlo for the vertex detector alone is illustrated by the $r-\phi$ resolution for first hits in axial cells, shown in Fig. 5(a), as a function of drift distance. This resolution is measured using triplets of next-but-one neighbouring hits to remove the effect of the stagger. Also shown, in Fig. 5(b), is the distribution of the difference in reconstructed drift distances between an axial 'second' hit and the preceding hit on the same wire. This is seen to be modelled quite well.

\subsection{Jet Chamber (CJ)}

\section{The detector geometry}

The jet chamber measures the trajectories of charged particles in a magnetic field over a solid angle close to $4 \pi$. A typical event containing jets with a large number of tracks close to one another requires good spatial and double-track resolution. Tracks are reconstructed as space points, using the wire position and the drift time to determine the transverse coordinates, and charge division to measure the position of the hit along the wire $(z)$. Multiple sampling of the ionization energy loss, $d E / d x$, of charged particles combined with the determination of their momenta allows the separation of different particle types. The signal pulse shapes are recorded at both ends of each sense wire and digitized by a readout system based on FADCs [15] with a sampling frequency of $100 \mathrm{MHz}$.

The jet chamber geometry $[16]$ is described by standard GEANT volumes. Inside a volume with an argon-methane-isobutane gas mixture at a pressure of 4 bar, there are conical aluminium plates carrying the cathode and anode wire supports and field shaping electrodes. The cathode wire supports are at the sector boundaries and the anode supports in the centre of the sectors. 
The sensitive volume of each sector is described by two identical trapezoidal volumes forming the halves of the sector symmetrical to its anode plane. Additional field shaping electrodes form the outer boundary of the jet chamber in the barrel region. Individual wires are not simulated in order to save computing time.

\section{Tracking and digitization}

GEANT extrapolates a track through the jet chamber and generates space coordinates. From these space points, the jet chamber simulation calculates the anode wires on which signals will be produced, and determines the positions with respect to the wires. These measurements are smeared to account for the detector resolution and for calibration effects. Parametrizations describing both detector resolution and calibration were obtained from previous experiments [17], the full-scale OPAL prototype [18] or the final chamber [16]. With the large amount of OPAL data available some of the parametrizations have been adjusted to improve the agreement between simulated and recorded events.

The result of the digitization step is a set of five numbers which fully describe each measured point - wire number, drift time, $z$-coordinate, integrated charge of the FADC pulse and a bit pattern describing the quality of the measurement. These form the input for calibration and pattern recognition procedures to reconstruct the jet chamber tracks, and correspond to the real data after the (online) analysis of the FADC pulses.

In a standard run of GOPAL all known resolution and noise effects and a selected set of calibration effects are simulated by default [19]. The following calibration effects are implemented:

- Effective mechanical staggering $\Delta_{\text {mech. }}= \pm 90 \mu \mathrm{m}^{4}$ : this is the distance by which the signal wires are staggered alternately to the left and right side of the anode plane in order to resolve left-right ambiguities.

- Electrostatic deflection $\Delta_{\text {es. }}^{\max }=+60 \mu \mathrm{m}$ and gravitational sagging $\Delta_{\text {grav. }}^{\max }=+200 \mu \mathrm{m}$. The $z$-dependence is approximated by parabolae having these maximal deflections at $z=0$.

- Lorentz angle $\alpha_{L}=20.0^{\circ}$ : this is the angle, measured from the perpendicular to the anode plane, at which ionization drifts to the signal wire.

- Drift velocity $v_{D}=52.7 \mu \mathrm{m} / \mathrm{nsec}$.

- Determination of the drift distance by approximating the trajectory of the first electron arriving at the signal wire ('JADE approximation').

- Effective wire length $l_{\text {eff. }}$ : this is the wire length used for charge division allowing for the influence of the wire resistance and the impedance of the preamplifiers.

These average values were measured with real data and are used in the standard simulation. They are independent of the sector number. The JADE approximation depends on the local track angle with respect to the signal wire plane. A more detailed description of these dependences can be obtained using the calibration part of the jet chamber simulation outlined below.

The resolution effects implemented in GOPAL are:

- Drift time resolution $\sigma(t)$. This depends on the length of the drift path (the distance between track and anode wire) and on the angle of the track with respect to the anode wire plane,

\footnotetext{
${ }^{4}$ Although the jet chamber was built with a staggering of $\Delta_{\text {mech. }}= \pm 100 \mu \mathrm{m}$ the measured effective staggering is reduced due to variations of the drift velocity in the region of the inhomogeneous field near the signal wires.
} 
$\Phi_{\text {local }}$. These dependences were parametrized using OPAL data. For $1.6 \%$ of all hits, the resolution is degraded to $14 \times \sigma(t)$ to take into account unresolvable $\delta$-rays.

- Double pulse resolution. The capability to resolve two close hits has been studied with real data and is parametrized as discussed in [20]. In accordance with data, hits start to be resolved at a separation distance of $1.9 \mathrm{~mm}$ with an efficiency that rises to $80 \%$ at a distance of $3.4 \mathrm{~mm}$. The resolution for the second hit is degraded up to $250 \mu \mathrm{m}$.

- Charge resolution $\sigma_{d E / d x}$ and $z$-resolution $\sigma_{z}$. The energy loss of a particle measured at an anode wire is evaluated according to the expectation obtained from real data [21], [22] and is smeared according to an experimentally determined Landau distribution. The resulting resolution for the truncated mean of all samples is $3-4 \%$. This resolution allows a $2 \sigma \pi-K$ separation for momenta up to $20 \mathrm{GeV} / c$. Afterwards, the charges on both ends of the signal wires are determined with charge division using the real $z$-coordinate. These charges are smeared with two normal distributions and are used to calculate the $z$-coordinate with an average $z$-measurement error of $1.0-1.5 \%$ of the wire length.

Noise effects and inefficiencies are also treated in GOPAL as follows. In accordance with OPAL data, the efficiency for finding a hit is set to $99.85 \%$. Noise in the chamber is simulated by generating hits not assigned to tracks. Such spurious hits may be correlated to real hits or are totally randomly distributed over the chamber. The former can arise from fluctuations of the FADC pulses or from low energy $\delta$-rays which are just resolved, and these effects are simulated by the generation of an additional $2.5 \%$ of all hits, which are delayed in time relative to real hits. Uncorrelated noise is simulated by the production of, on the average, 12 random hits per event to account for synchrotron radiation.

Optionally a more detailed simulation of some calibration effects may be used. In this part of the program [23] real measured calibration data are used, and additional effects may be simulated. It takes into account the field inhomogeneities in the jet chamber, inefficient wires due to missing calibration constants, different drift velocities for each sector etc., and enables the verification of the quality of the calibration procedures. Geometrical and mechanical effects include torsion of the cones, displacement of the anode planes and wire position. Time effects simulated include time offsets, signal propagation time along the wire and additional corrections due to the radial field near the signal wires. Charge effects include the track angle dependence of the measured charge, saturation originating in the screening of the electric field near the anode wire due to the remaining ions from the amplification of the first arriving electrons, attachment due to gas impurities, and dependence of the drift cell geometry on the staggering of the anode wires.

\section{Comparison with data}

Using $\alpha_{L}$ and $v_{D}$, the two-dimensional space point resolution $\sigma_{r \phi}$ results from the drift time resolution $\sigma(t)$. Figures 6(a) and (b) show the comparison of $\sigma_{r \phi}$, as determined by triplets of neighbouring wire measurements, as a function of the drift distance and of $\Phi_{\text {local }}$ in real data and Monte Carlo. Figure 6(b) is not symmetric about zero because of the non-zero Lorentz angle: $\sigma_{r \phi}$ is minimum for tracks perpendicular to the drift direction. Figure $6(\mathrm{c})$ compares the efficiency, in data and Monte Carlo, to resolve two hits as a function of hit separation.

A comparision of distributions relevant for the simulation of the specific energy loss is shown in Fig. 7. The average energy loss of a particle in the jet chamber is calculated by a truncated mean, in which some fractions of the lowest and highest of the measured $d E / d x$ values are rejected before calculating the mean energy loss. Figure 7(a) shows the distribution of the 
number of hits per track used for the calculation of the truncated mean. The dependence of the relative resolution on the number of hits used for the calculation of the truncated mean is shown in Fig. 7(b). Both distributions refer to multihadronic events with tracks in the polar angle region $|\cos \theta|<0.7$. The capability to separate different particle types using $d E / d x$ is indicated in Figs. 7(c) and (d). These distributions show the truncated mean for tracks in the momentum ranges $0.4-0.8 \mathrm{GeV} / c$ and $2.5-4.0 \mathrm{GeV} / c$ respectively. The agreement between data and Monte Carlo is generally satisfactory.

The good simulation of the space point resolution and the number of measurements on a track are the most fundamental elements in the quality of the simulation of the final reconstructed track parameters. The comparison of data and Monte Carlo of the final track parameters is deferred to section 3.5 .

\subsection{Z-Chambers (CZ)}

\section{The detector geometry}

The Z-chamber system is composed of 24 rectangular panels arranged as a cylindrical barrel aligned along the beam direction. Each panel is divided in $z$ into 8 drift cells, with an anode module at the centre supporting six staggered wires. The drift direction is along $z$. The geometry of these chambers is straightforwardly modelled using GEANT box and trapezoid shapes. In order to reduce the readout electronics needed the outputs from the 192 individual cells are multiplexed using the following scheme: an odd wire of a cell in the $p^{\text {th }}$ panel is multiplexed with its analogue in the corresponding cell of the $(p+4)^{t h}$ panel, and inside the same panel, an even wire of the $c^{t h}$ cell is multiplexed with its analogue in the $(c+4)^{t h}$ cell.

\section{Tracking and digitization}

Assuming each wire to be surrounded by a virtual drift space, hits are defined as the intersection of the GEANT track with the surface of these individual drift spaces. The tracking step inside the drift space is such that just the points of entry to and exit from the sensitive region are recorded. The final hits for each drift cell are then calculated using a quadratic interpolation based on the track momentum.

The digitization stage takes care of the left/right ambiguity, the panel/cell multiplexing and the double-hit resolution. The code generates calibrated digits (smeared space points) rather than uncalibrated digits (drift time and charge division).

The digitization proceeds in three steps. The first step starts from the GEANT hits, defining digits as the average position of the hits on either side of a drift cell, allowing for low-momentum tracks, dead wires, wire efficiency and real drift volume. Noise, if needed, is then superimposed before the smearing in the $y-z$ plane is performed and the left/right ambiguity is generated. The second step deals with the multiplexing, which leads to the generation of additional apparent hits. The digits are then sorted with respect to wire number and drift distance, and the double-hit resolution is finally introduced with its whole complexity inherited from the multiplexing scheme.

All parameters used in the digitization process are taken from (fits to) data. A constant $y$-resolution (along the wire length) $\sigma_{y}=1.75 \mathrm{~cm}$ is used. The $z$-resolution $\sigma_{z}$ is taken to be $z$ dependent, ranging from $100 \mu \mathrm{m}$ near the middle of any panel, to $300 \mu \mathrm{m}$ at its ends. The wire 
efficiency inside any cell, ranges from 0.980 for the innermost wires to 0.970 for the outermost ones. The double-hit resolution is $3 \mathrm{~mm}$.

\section{Comparison with data}

A precise measure of $z$ is particularly important in order to achieve good resolution on invariant masses using central detector tracks. Figure 8(a) shows the raw number of reconstructed segments in $\mathrm{CZ}$ as a function of $z$, for multi-hadronic events in data and Monte Carlo. The overall dependence on $z$, and thus $\theta$, is well reproduced. The internal resolution in $z$ is not perfectly reproduced by the Monte Carlo (Fig. 8(b)), but the discrepancy is within the acceptable limit. More important is the resolution in $r-\phi$, as it is a key variable in the association of the CZ segments to tracks in the jet chamber. Figure 8(c) shows the deviation of the $r-\phi$ measurement of $\mathrm{CZ}$ from the true position (given by $\mathrm{CJ}$ within a few hundred $\mu \mathrm{m}$ accuracy). These data are well reproduced by the Monte Carlo.

\subsection{Overall Performance of the Central Detector System}

For most physics analyses information from all components of the central detector is used in order to make the best estimate of track parameters. In this section we compare the results of the simulation of the drift chambers described in sections 3.2,3.3 and 3.4 above with OPAL data for combined tracks. This introduces new features, such as the extent to which the matching between different components of the system is reproduced by the simulation.

Figure 9 illustrates some global features of the tracks in multihadronic $\mathrm{Z}^{0}$ decays. Figure 9(a) shows the number of tracks per event (with some standard quality cuts imposed); Fig. 9(b) shows the momentum of individual charged tracks, and Fig. 9(c) shows the total momentum of all tracks in an event; Fig. 9(d) shows the track distribution in polar angle $\theta$. In all cases very good agreement is seen. However, in some cases the agreement is as much due to good tuning of the QCD event generator as to good detector simulation. More sensitive tests of the quality of the detector simulation are afforded by the distributions in Figs. 10 and 11.

In order correctly to simulate the resolution of the central detector system, it is necessary that the numbers of hits in each component be simulated realistically. We show this for multihadronic events in Fig. 10. In order for a track to be accepted for analysis we require at least 20 hits in the jet chamber (CJ), but no requirement of hits in the vertex chamber $(\mathrm{CV})$ or $\mathrm{Z}$ chambers (CZ) is imposed. Figure 10(a) shows that the number of hits in CJ is well modelled. Figure 10(b) shows the number of hits in CZ, where the comparison is restricted to the region $|\cos \theta|<0.68$ corresponding to the geometrical acceptance of the Z-chambers. The most important feature is that the number of tracks with no $\mathrm{CZ}$ hits is quite well described (though slightly underestimated in the Monte Carlo), indicating that the acceptance and matching efficiency are generally understood. Figures 10 (c) and (d) show the numbers of axial and stereo hits in CV. Again the agreement between data and Monte Carlo is reasonably good, though the simulation slightly underestimates the number of tracks in which no CV information is matched to the track. This is believed to be caused by residual systematic effects in the real data, for which allowance has to be made at the final analysis stage.

The resolution of the central detector is indicated in Fig. 11. Figure 11(a) shows the distribution of $d_{0}$, the distance of closest approach of a track to the main event vertex in the transverse $r-\phi$ plane, for tracks containing CV 'first' hits. The agreement between Monte Carlo and data is satisfactory. Figure 11(b) shows the same distribution on a wider scale, emphasizing the tails 
of the distribution which are governed not so much by the detector resolution as by physics processes in the Monte Carlo such as decays, interactions and multiple scattering. Figure 11(c) shows the corresponding impact parameter in the longitudinal direction, for tracks having both $\mathrm{CZ}$ and CV stereo hits - the agreement is less good because of systematic problems in the data mainly connected with $z$ reconstruction in the jet chamber, but still acceptable ${ }^{5}$. Figure 11(d) illustrates the momentum resolution (determined mainly by the jet chamber) in $\mu^{+} \mu^{-}$events.

\subsection{Time-of-flight barrel (TB)}

\section{The detector geometry}

The Time-of-flight (TOF) barrel scintillation counters are located immediately after the solenoid and before the barrel presampler and lead glass calorimeter. These counters are presently used for precision timing, (see e.g. Refs. [24], [25], [26]) and for triggering [8]. The geometry, readout electronics and trigger are described in Refs. [1], [8]. The 160 trapezoidal counters of $4.5 \mathrm{~cm}$ thickness, radius of inscribed circle of $234 \mathrm{~cm}$, inner base length of $0.89 \mathrm{~cm}$, outer base of $0.91 \mathrm{~cm}$ and of $684 \mathrm{~cm}$ length, cover the polar angle region $|\cos \theta|<0.82$ and all but $2 \%$ of the azimuthal acceptance (gaps between counters). They are read out at each end by photomultipliers (PM). The TOF detector was designed especially for particle identification at low momentum in multihadronic events, and as a trigger device. The geometrical description in GOPAL consists simply of 160 trapezoidal scintillator bars.

\section{Tracking and digitization}

For each particle tracked through the scintillators GEANT HITS are stored containing the first and last space-time coordinate of the particle trajectory in each bar, the corresponding energy loss, the path length in the bar, and kinematic information on the particle. The scintillation process, light collection, amplification and digitisation are simulated with a very much simplified model. The present simulation assumes that each hit gives rise to an equal amount of scintillation light emitted towards the left and right $( \pm z)$ PMs, this amount being proportional to the energy deposit $\Delta E$ of the hit in the scintillator ${ }^{6}$. The energy deposit is taken to be at the mid-point of the particle's path in the scintillator. The light is then attenuated in its propagation towards each PM (attenuation length about $230 \mathrm{~cm}$ ). The time taken for the light propagation is calculated using the effective light velocity in scintillator measured for these bars. This is an average over all the possible light trajectories and is close to that of a photon at the critical angle of the scintillator. The convolution of scintillator efficiency, light collection efficiency and photocathode quantum efficiency is accounted for by a single energy scale constant which is used to give the correspondence between observed photo-electrons and deposited energy. This constant is set to 50,000 photo-electrons per GeV for no attenuation (i.e. zero propagation distance in scintillator). In particular, this approach assumes that the light collection efficiency is uniform within the bar, and independent of angle of incidence.

In order to understand the approach used to simulate the digitisation, we first outline the detector readout. Each of the 320 analogue PM signals is passively split in two. A 12-bit ADC integrates the charge for one signal. The other signal is discriminated at a set threshold in a

\footnotetext{
${ }^{5}$ In fact, for many physics analyses, a technique is employed whereby tracks are constrained to the reconstructed main vertex in $z$; in this case the $z$-resolution is much improved, and also data and Monte Carlo agree much better.

${ }^{6} \mathrm{NE} 110$ is about $2 \%$ efficient in converting energy into scintillation light.
} 
constant fraction discriminator (CFD). The CFD output is digitised in 11 bit (50 ps/count) single hit TDCs and used in a mean-timer coincidence of left and right PMs. This coincidence is the basic trigger signal for each bar and the decision is also readout by 160 pattern unit (PU) channels. The ADCs, PUs and the trigger are gated with gates of about $150 \mathrm{~ns}$ around the beam crossing time. The TDCs are started $15 \mathrm{~ns}$ before the beam crossing.

In modelling the ADC, TDC and PU response, emphasis has been placed on using a method which takes into account multiple hits, which occur frequently for electromagnetic showers and hadronic jets. The unsmeared times of arrival and amplitudes at each PM for all hits in all bars are calculated as described above. The hits arriving within the specified gate are sorted in time order, and time packets are formed around the first hit in time which exceeds a small fraction of the CFD threshold. A time packet is a list of hits which are close in time to the initiator hit. Two different time packet widths are distinguished. Firstly, one with a width of several ns (similar to the width of a PM signal) which is the relevant time interval for determining if the discriminator fires, and secondly one with a width of a few hundred ps (similar to the rise time of the signals) which is taken to be the appropriate time interval to consider for the time output by the CFD and for assigning a certain amplitude to this timing. All amplitude smearing is proportional to $\sqrt{N_{p e}}$ where $N_{p e}$ is the amplitude expressed as the number of photo-electrons within the given time interval. The ADC response is equated to the smeared total number of photo-electrons. The discriminator fires if the smeared value of the amplitude within the long time packet exceeds a set threshold. For time packets where the discriminator does not fire, the time packet is redefined with the next initiator hit. If the short time packet after amplitude smearing exceeds the discriminator threshold, the time measured by the TDC is taken as the amplitude weighted time of the short time packet, smeared by the time resolution. If no such short time packet is found and the discriminator fires, the associated long time packet is used for the time response. The time resolution is taken to be solely due to photo-electron statistics. The PU response is equivalenced to both TDCs firing and the trigger response for each bar is taken to be the same as for the PU.

\section{Comparison with data}

Figure 12(a) shows the distribution of number of TOF bars where both discriminators fired in multihadronic events compared to the simulation. Figure 12(b) shows the distribution of $t_{0}$ (time measured - time expected for a photon) and Fig. 12(c) shows $z_{T O F}-z_{E B}$ where $z_{T O F}$ is obtained from the time difference between the two ends of the scintillator and $z_{E B}$ is measured in the barrel lead glass. Figure 12(d) shows the $t_{0}$ distribution for hit TOF bars in $\mu^{+} \mu^{-}$events. It can be seen that reasonable qualitative agreement is obtained, but that the time resolution is significantly worse in the data. The tails of the $\Delta z$ distribution are well simulated and this enables this variable to be used in rejecting multiple hits.

\subsection{Presampler Barrel (PB)}

\section{The detector geometry}

The barrel presampler is made of $1 \times 1 \times 600 \mathrm{~cm}^{3}$ gas cells with axial wires. The cells are arranged in layers of 96 cells wide. Each layer of cells has a plane of $1 \mathrm{~cm}$ wide strips oriented at $45^{\circ}$ relative to the wires on one side and another plane of strips oriented at $-45^{\circ}$ on the other side. A sector is two layers thick, and 16 sectors arranged in a cylindrical shell form the entire detector. The strips and the volume containing the gas cells are implemented in GOPAL as 
mixtures of their actual components in the shapes of phi sections of cylindrical shells which are sandwiched together to form a sector. The gas cells are inserted into the sectors as rectangular boxes filled with the gas used in the detector; the gas is defined as a mixture and as a sensitive volume.

\section{Tracking and digitization}

A particle passing through a cell ionizes a trail of gas molecules along its path. The wire collects the negative charge while the positive charge remaining in the cell screens the wire, reducing the sensitivity of the detector to other charge at the same longitudinal position along the wire [27]. The charge screens a greater length of the wire than the path of the particle projected onto the wire; this additional length is the dead zone. The strips capacitively sense the positive charge after the wire collects the negative charge.

The simulated wire signal is proportional to the charge deposited by the particle traversing the gas cell, to the observed variation of signal with the angle of incidence of that particle, and to the screening-shortened path length divided by the original path length. The amount of charge deposited by each particle is randomly generated according to a Landau distribution, whose mode is $6.7 \mathrm{keV}$ for minimum ionizing particles. The Landau distribution is truncated at $100 \mathrm{keV}$, and the GEANT tracking cutoff for the active volume and surrounding plastic is also set to $100 \mathrm{keV}$. The points where particles hit the gas cell are sorted by position along the wire and the projection of the path length along the wire together with the dead zone are calculated. The screening due to overlap between the paths of a pair of particles is accounted for by shortening the path length of each particle so that they no longer overlap. The average signal on a gas cell wire is parametrized as a function of the angle of incidence of the particle with respect to the wire and is taken to be constant near normal incidence, rising as the angle becomes more acute. The signal on the strips from a single particle passing through a gas cell is taken as the charge induced on strips of a conducting, grounded plane near a point charge of magnitude equal to the wire signal generated by the particle. The wire and strip signals from different particles are summed to give the total signal.

The dead zone is adjusted to fit the wire signal to that observed in Bhabha events in the OPAL detector; this value is consistent with that derived from tests on models of the presampler [27]. The parametrization of the average wire signal as a function of the angle of incidence of the particle is fitted to $\mu^{+} \mu^{-}$events. The distance of the point charge from the conducting plane used to calculate the strip signal is set to the middle of the gas cell with a $20 \%$ variation allowed from particle to particle to fit the variation observed in $\mu^{+} \mu^{-}$events. The efficiency of the detector is adjusted to fit $\mu^{+} \mu^{-}$events.

\section{Comparison with data}

One of the uses of the presampler is in electron identification. Because of showering in the magnet coil an electron will generally yield more particles in the presampler than a muon or hadron. In Fig. 13, we show the presampler multiplicity associated to tracks in various types of event. The multiplicity unit is the signal expected from one charged particle passing through the active gas volume of one layer of the presampler. Thus a single particle is expected to give a signal of about two multiplicity units if it passes through gas cells in both layers of the presampler, a signal of one multiplicity unit if it passes through a wall between cells in one layer and a gas cell in the other layer, and a no signal if it passes through a gap between sectors. In Fig. 13(a) we show the multiplicity associated to single tracks in $\mu^{+} \mu^{-}$events. The relative positions and amplitudes of the peaks at one and 2 multiplicity units and the shape 
of the high multiplicity tail are well modelled by the Monte Carlo. In Bhabha events, shown in Fig. 13(b), the multiplicity distribution is well reproduced by the simulation, particularly on the high multiplicity side of the peak. In Fig. 13(c) we show the multiplicity associated to single tracks in multihadron events. We see that the distributions are broadly comparable at low multiplicity, but the high multiplicity tail of the distribution is too high in the Monte Carlo. In all three types of events, the data show a greater inefficiency than the Monte Carlo, which is seen as the number of tracks with no associated multiplicity. This is not unexpected, because no attempt has been made to model the gradual loss of about $1 \%$ of the efficiency of the detector due to failing electronics during the course of the data taking.

\subsection{Electromagnetic Barrel Calorimeter (EB)}

\section{The detector geometry}

The barrel part of the electromagnetic calorimeter consists of a cylindrical array of 9440 lead glass blocks. It is located outside the magnet coil and covers the full azimuthal angle and the polar angle region $|\cos \theta|<0.82$. Each lead glass block is $\sim 10 \times 10 \mathrm{~cm}^{2}$ in cross-section and $37 \mathrm{~cm}$ long, corresponding to 24.6 radiation lengths. The longitudinal axes of the lead glass blocks point towards the interaction region. To achieve this geometry, blocks of 16 different shapes are used. In GOPAL they are represented by general trapezoids. The Čerenkov light produced by relativistic charged particles in the lead glass block is viewed by a phototube through a 4 or $6 \mathrm{~cm}$ long cylindrical light guide which is also made of the lead glass. Both the block and the light guide are defined as GEANT sensitive volumes.

\section{Tracking and digitization}

Charged particles traversing the lead glass produce Cerenkov radiation if their speed exceeds the threshold $\beta c=c / n$, where the refractive index of the glass $n$ is 1.847 at a wavelength of $587.6 \mathrm{~nm}$. Photons are emitted at an angle of $\cos \theta=1 /(\beta n)$ with respect to the direction of the charged particle. The angle approaches its maximum $\theta_{\max }\left(\cos \theta_{\max }=1 / n\right)$ for a highly relativistic particle $(\beta \sim 1)$. The average number of photons is proportional to track length and to $\sin ^{2} \theta$. The photons travel through the block and the light guide, undergoing repeated reflections on their surfaces. On the way some photons will be absorbed in the lead glass depending on their wavelength, or in the wrapping material around the block and the light guide. Some fraction of the photons eventually reach the photo-cathode of the phototube and are converted into photo-electrons according to the quantum efficiency which depends on the wavelength.

A program was developed which generates and tracks the Čerenkov photons in the counters. Tracking of the individual photons, however, consumes too much cpu time to be incorporated into standard GOPAL runs. A simpler and faster method was therefore adopted. For a track segment of a charged particle given to GUSTEP the average number of photo-electrons is calculated as a function of the position and the direction of the track segment. The function was determined for highly relativistic particles by using the program for full Čerenkov photon tracking. It is approximated by a function of only two parameters, namely, the angle of the track to the block axis and the distance from the front face of the block. The taper of the side surfaces of the block determines the reflection angles, thus affecting the detection efficiency of the photons, and therefore different parametrization functions are needed for the 16 counter types. In Fig. 14(a) the function is plotted for one particular type of counter. To account for 
a lower velocity of the track, the number of photo-electrons given by the function is multiplied by $\sin ^{2} \theta / \sin ^{2} \theta_{\text {max }}$ and this value is saved in the GEANT HITS structure. However the effect of a smaller emission angle is not taken into account.

At the digitization stage the contributions from all the track segments in the block and the light guide are summed up for each counter. The total number of photo-electrons is then linearly scaled to an energy value. Since the gains of the real counters were calibrated with a $50 \mathrm{GeV}$ electron beam, the conversion factor was derived by running GOPAL with $50 \mathrm{GeV}$ electrons incident on the block without material in front. The energy value does not include the statistical fluctuations in the number of photo-electrons or those in the amplification process of the phototube. These effects are taken into account by artificially smearing the energy with a Gaussian distribution. The size of the smearing was determined from the observed electron energy resolution of real counters.

\section{Comparison with data}

An electromagnetic shower deposits its energy in a cluster of lead glass blocks. The lateral spread is largely determined by multiple scattering in the lead glass and in the material in front. Figure 14(b) shows the fraction of the cluster energy which is contained in the most energetic block for high energy electron clusters in barrel Bhabha events. The average of this fraction is plotted as a function of the azimuthal angle of the cluster centre measured from the middle of the block. Though the shower spread is systematically smaller in Monte Carlo clusters, the level of agreement is satisfactory.

Since the energy normalization is based on electrons, critical tests of simulation performance can be done by observing the response to particles other than electrons. Figure 14(c) and (d) show the energy and the number of blocks contained in individual electromagnetic barrel clusters for multihadron events at the $\mathrm{Z}^{0}$ peak. The Monte Carlo distributions are generally in good agreement with the real data up to the higher tail regions.

\subsection{Presampler Endcap (PE)}

\section{The detector geometry}

In the space between the central detector pressure vessel and the endcap electromagnetic calorimeters, two presampler detectors [28], built of high gain multiwire proportional chambers, are installed in an umbrella-like arrangement. The presamplers provide measurements of the shower development following roughly 1.6 radiation lengths of material, in the angular range $0.83<|\cos \theta|<0.95$. Each of the presampler detectors is divided into 16 trapezoidal sectors, consisting of a large chamber covering the angular range $0.83<|\cos \theta|<0.92$ and a small chamber covering the range $0.90<|\cos \theta|<0.95$. The large chambers are read out via pads of variable size $\left(\approx 9-18 \times 22 \mathrm{~cm}^{2}\right)$ for two-dimensional readout, with $\approx 1.1-2.2 \mathrm{~cm}$ wide strips for measuring the azimuthal angle, and groups of 4 wires giving a $0.8 \mathrm{~cm}$ pitch for the determination of the polar angle. The small chambers are read out by horizontal and vertical strips. The total number of electronics channels in both detectors is 6080 . The geometry is modelled by GEANT polycone and box shapes.

\section{Tracking and digitization}

The chambers work in a nearly saturated mode [30], such that the total pulse height observed is roughly proportional to the number of shower particles entering the chamber. A particle 
traversing the $0.32 \mathrm{~cm}$ thick active layer of the detector ionizes gas molecules along its path. After amplification, the negative charge is collected by the wires and the image charge is seen divided between the cathode pads and strips. In the Monte Carlo, each particle that traverses the PE sensitive region during the GEANT tracking leaves a hit in a detector element.

The response of the detector to minimum ionizing particles has been calibrated in test beam measurements and adjusted using the data. It is parametrized as the sum of two Gaussian distributions. In GOPAL the signals left by the shower in the detector elements are then simulated by adding up the energy depositions of all GEANT tracks hitting these elements.

\section{Comparison with data}

The presampler detector can be used to discriminate between minimum ionizing particles and those that have hadronic or electromagnetic interactions in the preceding material. In Fig. 15(a), the pulse-height distribution of muons detected by the endcap presampler is plotted in units of the signal expected from minimum ionizing particles. A similar distribution for single track $\tau$ decays is shown in Fig. 15(b). The agreement between Monte Carlo and data is satisfactory.

\subsection{Electromagnetic Endcap Calorimeter (EE)}

\section{The detector geometry}

The electromagnetic endcap calorimeter [29] consists of 2264 lead glass blocks instrumented with VPTs, covering the angular range $0.81<|\cos \theta|<0.98$. The blocks, of cross-sectional area $9.2 \times 9.2 \mathrm{~cm}$, and length $35,38,42$ or $52 \mathrm{~cm}$, are encased in $0.5 \mathrm{~mm}$ thick brass cans supported by a rigid peralumin backplate. They are arranged in a rectangular grid, with their axes parallel to the beam axis. In GOPAL each assembly is simulated by a block of lead glass surrounded by an air gap and a brass can, together with material representing the VPT and associated electronics. All materials are defined using the GEANT mixture routine. Because of the simple rectilinear geometry, the GEANT user search facility is employed during tracking to determine which volume a point lies in.

\section{Tracking and digitization}

Charged particles in the lead glass produce Čerenkov radiation, which is detected by the photocathode of the VPT glued to the end of each block. The number of Čerenkov photons emitted is proportional to track length and to $\sin ^{2} \theta$ where $\theta=1 /(\beta n)$ is the Cerenkov angle, $\beta$ is the particle velocity and $n$ the refractive index of the glass. The fraction of the photons which reach the photocathode and are detected as photoelectrons depends sensitively on the position and angle of the track with respect to the block, the properties and geometry of the lead glass, and the size and quantum efficiency of the photocathode. The mean detected number of photoelectrons per unit track length for a highly relativistic particle has been parametrized as a function of the angle of the track to the block axis and of the distance from the photocathode. During tracking, for each charged particle step in a lead glass block, the mean number of photoelectrons is obtained from the parametrization, multiplied by $\sin ^{2} \theta / \sin ^{2} \theta_{\text {max }}$, where $\theta_{\max }$ is the Čerenkov angle for a highly relativistic particle. Charged particles are tracked down to a cutoff equal to the Cerenkov threshold in the lead glass, $121 \mathrm{keV}$. At the end of tracking, the number of photoelectrons is summed for each block, and converted to energy using calibration factors derived by running GOPAL with $20 \mathrm{GeV}$ electrons injected into the centre of the front 
face of a block - a procedure which precisely simulates the calibration of the detector in an electron beam. Finally, random noise, from a Gaussian with an r.m.s. of $14 \mathrm{MeV}$ as determined from data, is added to each block, including those with no other signal. The energy in groups of blocks is then summed to simulate the trigger signals.

The parametrizations are obtained by injecting high energy muons into a lead glass block in GOPAL. For each track segment, Čerenkov photons are generated and tracked through the block, taking account of reflections at the surfaces, absorption (using known absorption coefficients as a function of wavelength) and the size and wavelength-dependent quantum efficiency of the photocathode. The mean number of photoelectrons detected is then parametrized as a function of distance from the photocathode and angle to the block axis, using a 4 th order polynomial in distance in each of 40 angular bins. Figure 16(a) shows a plot of this function. Variations with other parameters, such as lateral position, are less important, and tend to average out in an actual shower. Results using the parametrization agree with those using full Čerenkov photon tracking to about $1 \%$ for electron and hadron showers over a wide range of energy.

\section{Comparison with data}

The lead glass simulation was extensively tested against test beam data before LEP startup, and comparison with OPAL data is generally very satisfactory. The overall energy normalisation depends on correctly simulating the response to particles incident at an angle to the block axis, and also on correct simulation of material in front of the lead glass. Figure 16(b) shows the cluster energy divided by beam energy for the two highest energy electron clusters in Bhabha events, in the region $0.85<|\cos \theta|<0.9$ where there is least material in front of the lead glass. The overall normalization is good to better than $1 \%$ although the energy resolution is slightly better in Monte Carlo than data, but the excess of events with low energy in the data is probably due to a small residual contamination from $\tau$ pairs. Figure $16(\mathrm{c})$ shows the total energy in the endcap lead glass in multihadronic events, and the agreement between Monte Carlo and data is excellent. A more stringent test of the simulation is the lateral development of electromagnetic showers, which is determined to a large extent by multiple scattering, and thus depends on the properties of the lead glass being simulated correctly. Figure 16(d) shows the mean fraction of energy in the block with most energy, $f_{\text {max }}$, for the two highest energy electron clusters in Bhabha events as a function of angle. It can be seen that in the Monte Carlo the showers are slightly too narrow, as shown by the systematically low value of $f_{\max }$, but the variations in shower shape with angle are well reproduced.

\subsection{Hadron Barrel and Endcap Calorimeters (HB/HE)}

\section{The detector geometry}

The OPAL hadron calorimeter consists of a barrel module with 24 wedges in $\phi$, extending to $|\cos \theta|=0.81$ in polar angle, two endcap modules in the range $0.81<|\cos \theta|<0.91$, and two poletip modules in the range $0.91<|\cos \theta|<0.99$. The simulation routines for the barrel and endcaps are similar, the poletips are discussed separately below (section 3.12).

The barrel and endcap calorimeters consist of $10 \mathrm{~cm}$ thick iron slabs interleaved with wire chambers operating in the limited streamer mode. The barrel has nine active layers and the endcap eight. Particles traversing these streamer tubes induce a signal on pads of size $50 \times$ $50 \mathrm{~cm}^{2}$ under the tubes, and on $1 \mathrm{~cm}$ wide strips above each tube, running parallel to the signal 
wires. The pads are arranged in towers and their analog signals are summed before they are read out, whereas for the strips the presence of a pulse above threshold is registered as a binary bit.

The geometrical description of the hadron calorimeter in GOPAL contains the iron as passive material and the gaps between the iron slabs as active detectors; the streamer tubes are not positioned individually to make the GEANT tracking faster.

\section{Tracking and digitization}

During the tracking phase of GOPAL, the coordinates of all charged particles traversing the active layers of the calorimeter and the lengths of the tracks in a $1 \mathrm{~cm}$ thick layer representing the tubes are stored. In the digitization phase, the hit coordinates are retrieved and the following procedure is used for each wedge:

- The chambers are assumed to consist of rectangular tubes separated by $1 \mathrm{~mm}$ walls. No signals are generated for particles traversing these walls.

- Digits are generated for each strip corresponding to a traversed tube. Particles traversing more than one tube give a digit for each strip individually.

- Particles with overlapping trajectories through the same tube are replaced by one particle following the sum of the trajectories.

- The streamer charge produced by the charged particle is calculated with a parametrization obtained from a test beam measurement of penetrating muons. The distribution of charges $p(Q)$ is represented by the sum of a Gaussian distribution with mean $\bar{Q}$ and width $\sigma_{Q}$ and a Landau distribution $p_{L}$ with the same mean and width:

$$
p(Q)=f_{G} \exp \left(-(Q-\bar{Q})^{2} / 2 \sigma_{Q}^{2}\right)+f_{L} p_{L}\left(2(Q-\bar{Q}) / \sigma_{Q}\right) .
$$

The contributions of the two distributions $f_{G}$ and $f_{L}$ and the mean and width are functions of the incidence angle of the particle on the tube; they are constant for $\lambda<20^{\circ}$, where $\lambda$ is the angle between the particle direction and the normal to the tube. Above $20^{\circ}, f_{G}, \bar{Q}$ and $\sigma_{Q}$ rise linearly with $\tan \lambda$ until saturation is reached.

- The induced charges for each tower are added and converted into digits.

- Additional strip hits are added due to crosstalk. This crosstalk is a function of the streamer charge and parametrized from the test beam measurements.

\section{Comparison with data}

The absolute hadronic energy scale is also derived from test beam measurements: the pad signal of a penetrating muon at normal incidence corresponds to the signal of a $2 \mathrm{GeV}$ pion at normal incidence. The calibration of real data uses this relation and the measured charges from $\mathrm{Z}^{0} \rightarrow \mu^{+} \mu^{-}$events. Figures 17 (a)-(c) show the reconstructed hadronic energy for penetrating muons from $Z^{0} \rightarrow \mu^{+} \mu^{-}$events for different angles of incidence. Figure 17 (d) shows the total observed hadronic energy for multihadronic $\mathrm{Z}^{0}$ decays, compared with the prediction from the JETSET 7.2 generator. The agreement is generally satisfactory. 


\subsection{Hadron Pole Tip Calorimeter (HP)}

\section{The detector geometry}

The hadron pole tip calorimeter consists of one calorimeter in each endcap covering the angular range $0.88<\cos \theta<0.98$, and the full $360^{\circ}$ of azimuth. The calorimeter is comprised of 10 layers of thin gas chambers interspersed with iron. In each layer there are 16 counters each of which spans 22.5 degrees in azimuth. Each of these counters contains sensitive area which is segmented into pads on one side of the chamber and radial strips on the other side. A charged particle traversing the sensitive area ionizes the gas in the chambers and the negative charge is collected on anode wires within the chamber. The induced charge is picked up by the pads and strips and is read out. The pad readout is used to locate the shower in $\theta$ and $\phi$ and the energy readout is grouped in towers where each tower consists of the pads in all layers in a given $\theta$ and $\phi$ region. The strips give finer azimuthal information and sample each layer. Whereas the response of the towers is almost linear in energy with a resolution varying from 100 to $140 \% / \sqrt{E}$, the strip readout produces a response independent of energy for a given strip. The azimuthal angular resolution of the pads is about 0.1 rad whereas that of the strips is about 12 mrad. The polar angular resolution of the pads is about $0.07 \mathrm{rad}$.

\section{Tracking and digitization}

Particles traversing the pole tip calorimeter are treated in GOPAL as follows: Each tower hit is converted to the equivalent energy deposited by a minimum ionizing particle (as determined from results of a test beam) which is then smeared with a Gaussian distribution so as to agree with the known resolution of the calorimeter. The energy recorded is the equivalent energy of the sum of all hits in a given tower. The value of this energy and the tower descriptor (end cap number, azimuthal region and pad number) are then stored as a 32 bit packed word which is later used by the reconstruction program. The information from the strips consists of the descriptor of the first strip fired in a given chamber and the number of consecutive strips fired. This is also stored as a packed 32 bit word.

\section{Comparison with data}

We have compared the GOPAL prediction for the energy response of the pole tip calorimeter to a sample of OPAL data containing multihadronic events. We consider here the numbers and energies of clusters, where a cluster basically includes contiguous hit pads. The results are shown in Fig. 18 for the total energy of all clusters, the number of clusters per event and the energy in a cluster. We see fairly good agreement between the data and GOPAL.

\subsection{Muon Barrel (MB)}

\section{The detector geometry}

The muon barrel subdetector (MB) consists of 110 large-area drift chambers mounted in four layers on the outside of the hadron calorimeter. Each chamber consists of two drift cells with a maximum drift distance of $29.7 \mathrm{~cm}$. The chambers are $10.4 \mathrm{~m}$ in length over most of the detector; shorter, $8.0 \mathrm{~m}$ and $6.4 \mathrm{~m}$, chambers are required to fit between the support legs. The hit $z$ coordinate is obtained from a system of diamond-shaped cathode pads [31]; a coarse$z$ measurement is obtained using charge division on the wire, a medium- $z$ measurement is obtained from charge division using pads with a $171 \mathrm{~cm}$ wavelength and a fine- $z$ measurement 
is obtained from charge division using pads with a wavelength of $17.1 \mathrm{~cm}$. Using these three values the wavelength ambiguities can be resolved and space points can be obtained with a resolution $\approx 2 \mathrm{~mm}$ in each direction.

The MB geometry is defined as a tree structure using the GEANT geometry package. At the top level is a cylindrical mother volume which encompasses the MB subdetector and is positioned in the global OPAL volume. Below this are the 110 chambers. These consist of the chamber "frame" which is trapezoidal in cross-section and is positioned within the mother volume using the survey data (obtained either from default values or from the database). Within each such frame the sensitive volume of the chamber is defined as a rectangular box. The material within each chamber is defined using an average over the volume including the chamber gas and additional material.

\section{Tracking and digitization}

Any track which traverses the MB sensitive volume during the GEANT tracking phase produces a hit which is stored. During the digitization phase the ambiguity hits are added and Gaussian smearing is applied to the hit positions to allow for the chamber resolutions. The chamber efficiencies and the production of spurious noise hits are simulated on a chamber by chamber basis using values derived from the data. The medium and fine $z$ values are obtained from the $z$ value modulo the wavelength ambiguities and the coarse, medium and fine $z$ values are smeared independently. If two hits occur within $2 \mathrm{~cm}$ in the drift direction then they are replaced with a single hit (with an increased smearing) to account for the chamber two-hit resolution. The MB chamber efficiencies, the chamber noise and the chamber resolutions were initially obtained from chamber test setups. Since the start of LEP operation these parameters have been tuned to give good agreement with the data.

\section{Comparison with data}

In the event reconstruction, muon candidates are found by first fitting straight track segments to the muon chamber hits in order to resolve the hit ambiguities. Tracks found in the central detector are extrapolated to the muon chamber radius and are then matched using both position (i.e. the displacement between the centre of gravity of the muon segment and the position of the extrapolated track at the same radius) and direction (i.e. the angle between the muon segment and a tangent to the extrapolated track). The matching in both $\theta$ and $\phi$ is shown in Fig. 19 for data taken during the 1990 running period and for the GOPAL simulation. The resolution in the data can be seen to be somewhat worse than in the Monte Carlo, particularly in the direction matching. This is due to systematic uncertainties in the survey data of order 2-3 $\mathrm{mm}$ which are not included in GOPAL.

\subsection{Muon Endcap (ME)}

\section{The detector geometry}

The muon endcap chambers consist of planes of limited streamer tubes. The area is covered as completely as possible by means of rectangular quadrants and patches, which are in turn constructed of modules in subdivisions of units, each of 8 gas tubes [32]. These are modelled in GOPAL by box-shaped volumes. Just as in the real structure, gas tubes are placed in plastic units and built up into modules, quadrants and patches. Each is placed in its design position, which corresponds closely to the final structure, particularly in respect of the active area covered. 


\section{Tracking and digitization}

When a charged particle passes through a gas tube, it causes a limited streamer discharge which induces charge on detection strips placed on either side, on one side parallel to and on the other side perpendicular to the tubes. Detection of this charge thus gives both $\mathrm{x}$ and $\mathrm{y}$ coordinates. The discharge and response is modelled in GOPAL by choosing the central pulse height at random according to the distribution observed experimentally, of the form $x e^{-a x}$, where $a$ is a fitted constant; for the perpendicular strips, the pulse height is lower than on the parallel ones by a factor of 0.55 with a $\pm 40 \%$ variation. Relative to the central height, the adjacent strips are set according to a Gaussian distribution with each value smeared at random by a further $\pm 20 \%$. The Gaussian width for the parallel strips is $2 \mathrm{~cm}$. For the perpendicular strips, the width of the central peak is $1 \mathrm{~cm}$. In addition there is a background of width $10 \mathrm{~cm}$ added with $20 \%$ of the central height. All of these parameters were chosen by fitting the data observed experimentally. For very short path lengths, real data indicate some cut-off in the streamer response and it has been necessary to tune this parameter. The output data stored consist of strip identification and pulse heights.

\section{Comparison with data}

Muon pair events provide a suitably clean sample of muons and have been chosen for the comparison of Monte Carlo and real data, taking samples of $10000 \mu^{+} \mu^{-}$events from GOPAL and over $9000 \mu^{+} \mu^{-}$events from the 1991 OPAL data. A good test of the reliability of the simulation is in the total number of track segments reconstructed per muon. Figure 20(a) shows that the data and Monte Carlo distributions are comparable, though with rather less 1-segment and more 2-segment muons in the real data. This is thought to be due to noise, for example cosmic tracks, which are not modelled in the Monte Carlo program and which will give rise to a shift in this direction.

In each $\mathrm{x}$ and $\mathrm{y}$ projection there are typically 4 hits per segment, corresponding to the 4 measurements of each coordinate; this can go up to 8 hits where chambers overlap. Figure 20(b) compares data with Monte Carlo for the $x$ projection (that for $y$ being effectively identical). From these distributions it is possible to estimate the streamer tube plane efficiency; for example, the ratio of 3 -hit to 4 -hit segments is given by $4(1-\epsilon) / \epsilon$, where $\epsilon$ is the single plane efficiency. When allowance is made for those tubes known to be not working due to high voltage problems $(1.3 \%$ ), we find $\epsilon$ to be $93.9 \pm 0.3 \%$ for real data compared to $96.2 \pm 0.2 \%$ for Monte Carlo. This discrepancy indicates that some further slight tuning of the short pathlength cutoff parameter is necessary. However, since there is considerable redundancy of hits in finding segments, the overall efficiency for this is given by $\left(1-2(1-\epsilon)^{2}\right)$, or $99.25 \%$ for real data and $99.72 \%$ for Monte Carlo, a discrepancy of less than $1 \%$.

\subsection{Forward Detector (FD)}

\section{The detector geometry}

The OPAL forward detector consists of several subdetectors covering the extreme forward regions, at polar angles $(\theta)$ between 45 and $250 \mathrm{mrad}$. Their primary purpose is to determine the luminosity by detecting electrons and positrons from small-angle Bhabha scattering, with errors comparable with the statistical errors on the $\mathrm{Z}^{0}$ decay channels. This requires a determination of the effective cut at small $\theta$ to better than 0.25 mrad.

The forward subdetectors implemented in GOPAL are 
- The main calorimeter, FK, and presampler, FP which together measure the total energy of electromagnetic showers, and also provide the forward detector triggers. The presampler consists of six radiation lengths of lead-scintillator calorimeter, divided into 16 segments in $\phi$, and six layers in $\mathrm{z}$, and the main calorimeter consists of a further 33 layers with similar structure. Scintillation light from all $z$ layers in the same $\phi$ segment is transmitted to a photomultiplier via a light guide connected to the outer edge of the scintillator. The main calorimeter also has light collection from the inner edges, and so measures $\theta$ (determined by the ratio of inner to outer signals) and $\phi$ (determined by ratio of signals in adjacent segments)

- The forward tube chambers FB. Three planes of tube chambers placed between the forward presampler and the main calorimeter, used for accurate position measurement of shower centroids.

- The fine luminosity scintillator counters, FL, positioned in front of the calorimeters, used to give an independent measurement of luminosity. Each quadrant contains a pair of trapezoidal scintillators, one A (acceptance) counter and one slightly larger $\mathrm{C}$ (coincidence) counter. A Bhabha event in the FL counters may be identified by a coincidence of one $\mathrm{A}$ and both $\mathrm{C}$ counters in a pair of diagonally opposed quadrants (a telescope).

\section{The Forward Calorimeter}

Since the calorimeter provides the trigger for all Bhabha events, an accurate calorimeter simulation is required for any luminosity analysis. The response of the calorimeter in GOPAL is determined by three processes :

1. The development of showers in the beam pipe, pressure vessel window, detector support structures and the calorimeter itself.

2. The deposition of energy in the scintillator layers, and the collection of light by the scintillator readout.

3. The response of the electronics (photomultipliers and ADCs) to the light collected.

The shower development process may be optimized by setting parameters to control the tracking algorithms in GEANT. In particular, it was necessary to use a small minimum step length in the beam pipe, low energy cutoffs in the calorimeter ( $1 \mathrm{MeV}$ in lead, $2 \mathrm{MeV}$ in the scintillator), and a small step length for multiple scattering in lead. These parameters determine the transverse spread of the showers, which in turn controls the sharing of energy between adjacent $\phi$ segments of the calorimeter. An underestimate of the transverse shower spread can be recognized by a depletion of events near the boundaries between segments, and an excess at the centre.

The energy deposited in each scintillator layer is calculated by GEANT, but the collection of scintillator light has to be determined empirically. This is done by fitting functions $F_{\text {in }}(\theta, \phi)$ and $F_{\text {out }}(\theta, \phi)$ which represent the response of a single scintillator layer to energy $E$ deposited at a point $(\theta, \phi)$, so as to reproduce the response of the entire calorimeter (in which the output is summed over $z$ ). The functions $F_{\text {in }}, F_{\text {out }}$ are similar to the functions representing the response of the entire calorimeter, but with additional exponential terms to represent light loss in the scintillator. The inputs to the fit are: test beam data taken at known $(\theta, \phi)$ values, histograms of energy deposited vs $(\theta, \phi)$ from Monte Carlo runs using a single electron generator run at each test beam point, and the relationship between $E_{\text {clus }}$ (the cluster energy) and $\Theta_{\text {clus }}$ (the measured theta coordinate) observed in real OPAL data. A reasonable fit can be obtained for 
angles above $45 \mathrm{mrad}$. At smaller angles showers are incident directly on the inner surface of the calorimeter, without passing through the presampler.

The ADC and photomultiplier response is represented by an overall gain factor which converts the sum of energy deposited in the scintillators to an ADC count for the corresponding channel. To this is added a pedestal, with mean and standard deviation corresponding to a typical value from the real data.

In Fig. 21 we show some comparisons between GOPAL and data for Bhabha events. Except for a very small number of radiative events, these contain collinear electrons and positrons with equal energy. The cluster energy distribution (Fig. 21(a)) (with the region below $50 \mathrm{mrad}$ in $\theta$ excluded to remove showers not traversing the full length of the calorimeter) agrees very well. In Fig. 21(b) we show the difference in energy between the left- and right-hand detectors - the width of the distribution is a measure of the energy resolution. Likewise, Fig. 21(c) shows the difference in $\theta$ between the two ends. Good agreement may be observed. Table 2 compares the resolution of the forward calorimeter determined from GOPAL with that obtained from OPAL 1990 data, calculated from the standard deviation of the difference between quantities measured by the left and right hand subdetectors for Bhabha events. No serious disagreements are observed.

\section{The Forward Tube Chambers}

The tube chambers consist of three planes (horizontal, vertical and diagonal) of 32,33 and 44 tubes each. However, they are represented in GOPAL by three disks of sensitive material (to reduce the time taken during tracking), and each hit is assigned to a particular tube during digitization. Since the tube chambers are situated after six radiation layers of presampler, the shower profile is largely determined by the representation of the calorimeter material, and no further optimization was necessary. A very low energy cutoff $(10 \mathrm{keV})$ is required in the tube chamber gas, since otherwise the energy deposited by particles passing through the tubes is entirely dominated by the small number of stopping particles. The response of the tubes to the energy deposited is adequately described by a scale factor and an appropriate pedestal, the position determination being based entirely on the known position of the tubes. In Table 2 the resolution in $\theta$ and $\phi$ for real and Monte Carlo Bhabha events is shown.

\section{The Fine Luminosity Counters}

The fine luminosity counters are modelled in GOPAL as four pairs of trapezoidal scintillators at each end of OPAL. The simulation uses the nominal counter positions, and light guides and photomultiplier tubes are not included. The ADC output from each counter was calculated by multiplying the energy deposited by a light collection function, then applying typical pedestal and gain factors.

A simple comparision of the Monte Carlo with real data is given by comparing the number of accepted Bhabha events with different combinations of $\mathrm{A}$ and $\mathrm{C}$ counters. Table 3 shows the result of this comparision, after normalizing to equal numbers of $A C C$ events. There is good agreement in all categories. The GOPAL Monte Carlo is important in determining the effect of scattering and showering in the beam pipe (with associated bellows, flanges and support rings ) in front of the scintillators, and so an accurate representation of these components is required to reproduce the effects of preshowering and multiple scattering observed in the data. 


\section{The Fast "SMEAR" Mode of GOPAL.}

For many applications in physics analysis it is desirable to have a much faster version of the Monte Carlo program, in order to be able conveniently to assess the effect of changing physics parameters or parameters governing the detector response. OPAL has such a fast simulation program, which runs as part of GOPAL, but using a much simplified form of the GEANT geometry and tracking. One advantage of working in the GOPAL framework is that the same utilities, such as interfaces to event generators, are available as in full simulation. A further benefit from running the fast simulation in the GOPAL framework is that one can mix the full simulation for one part of the detector with the fast version for another part. The fast simulation eventually fills the normal OPAL DST structure so that the same physics analysis code can run on either full or fast Monte Carlo output, and on data. The user can write a DST from a simulation run in the smear mode, or more commonly will run the analysis code inside the GOPAL job, using the DST structure in memory, and write out histograms and N-tuples.

We use standard GEANT tracking through a much simplified geometrical representation of the central detector. Thus decays, photon conversions, multiple scattering, bremsstrahlung, hadronic interactions etc. are all generated using the same GEANT code as in full simulation. However, a few mechanisms (such as $\delta$-rays) are disabled, and tracking cutoffs and tracking parameters are tuned to improve tracking speed. During tracking the points at which charged particles enter and leave the sensitive central tracking regions are recorded. These are used as the inputs to a procedure for smearing the ideal track parameters, which we outline here:

- The key ingredient is a careful estimation of the numbers and radii of the hits in each component of the central drift chambers.

- We work with "superpoints", each representing several real wires. There are 16 superpoints in the main jet chamber (compared with 159 wires) and 2 each in the vertex and Z-chambers (18 and 6 wires respectively).

- Two-hit resolution is applied at the level of the superpoints.

- Inefficiencies for the matching between tracks found in the jet chamber and the vertex and Z-chambers are allowed for, and we account for the possibility of tracks being split when they cross anode or cathode planes in the jet chamber.

- Single-hit efficiencies are applied.

- From the numbers of hits surviving, and using values for the resolutions in each detector (parametrized as a function of drift distance in the case of the $r-\phi$ plane) we compute the expected covariance matrix of the track parameters. The ideal track parameters are then randomly smeared using this covariance matrix.

- The numbers of hits suitable for calculation of $d E / d x$ are calculated based on the number of jet chamber hits, applying a further isolation requirement, and from this a smeared value for $d E / d x$ is computed.

- The primary vertex position and secondary vertices are reconstructed by running the normal OPAL vertex-finding algorithm. 
This fast version of the central detector simulation works well, and is particularly valuable for analyses where only charged tracks are used (e.g. some lifetime studies, or charm tagging using the $D^{*} \rightarrow D \pi$ decay chain). In particular the user can change resolutions easily and quickly check the effect on his analysis. Figure 22 shows a comparison between the smear mode of GOPAL and data for some typical distributions which could be used in a lifetime analysis. The agreement is good. The main problem with this fast simulation is that the parameters of the smearing algorithm have to be retuned as and when improvements in the real track reconstruction appear.

The simulation of the calorimeters and the outer muon detectors in the fast mode of GOPAL works through a crude tracking of "showers" through the detectors. Each particle entering the outer region is tracked through a highly simplified representation of the detector geometry, with parametrizations for the energy deposited in each sensitive detector including some correlations between the hadronic and electromagnetic parts. The simulation operates at the cluster level in the calorimeters. This allows an acceptable simulation for single isolated particles, but the combination of such clusters when they overlap in a multiparticle environment such as a jet is extremely difficult. The fast calorimeter simulation is therefore not nearly as satisfactory as that for the central detector. Nonetheless, it can still be useful when looking at trends, though one generally needs the full simulation to obtain reliable absolute results.

The smear mode of GOPAL runs typically about 100 times faster than the full simulation. Approximately half the time is spent in tracking, so this places an upper limit on the overhead imposed by running in the GEANT framework. Typically $10-20 \%$ of the time is spent in the event generator and setting up the TREE and the GEANT kinematics, and the remaining time is spent in the smearing code and creating the DST data structure.

A further option is to run the program in "No Detector" mode. In this case the particles from the event generator are copied directly to the DST banks, with no detector simulation nor any smearing of parameters.

\section{Summary}

The GOPAL Monte Carlo program, based on the GEANT package, has been reviewed. The OPAL collaboration has long been committed to GEANT, and several members of OPAL have contributed to the writing of GEANT. Generally our experience with GEANT has been good. Clearly we benefit from the fact that many physicists around the world are using and checking the package.

The GOPAL Monte Carlo has been an invaluable tool in preparing the OPAL software for LEP start up. Since then efforts have generally been directed at understanding the data, and in many cases the result has been to bring the data into closer agreement with the expectations from the Monte Carlo. Now, some two years after the first data, we are just returning to the task of making the simulation more realistic. A snapshot of the current performance of the program is represented by the figures in the present paper. Many illustrations of the use of the Monte Carlo in physics analysis can be seen by referring to the OPAL publications.

The close integration of the simulation and reconstruction codes has been most important in allowing the Monte Carlo to be used effectively in analysis. The fast simulation option works well for the central detector, though it is more problematic for the outer detectors. The 
close integration between the fast and full simulation modes is advantageous, since it provides a uniform framework for physics generators, tracking and analysis routines. Some features of the program, such as interactive use and graphics, were more important during the earlier development stages of the program, while new features such as the multi-cpu parallel processing mode have become increasingly valuable in a production environment.

\section{Acknowledgements}

We thank the many members of the GEANT team in the CN division of CERN who have maintained the GEANT package, and assisted us in other ways. We also acknowledge the contribution of many other past and present members of the OPAL collaboration.

In addition to the support staff at our own institutions we are pleased to acknowledge the Department of Energy, USA, National Science Foundation, USA, Science and Engineering Research Council, UK, Natural Sciences and Engineering Research Council,Canada, Israeli Ministry of Science, Minerva Gesellschaft, Japanese Ministry of Education, Science and Culture (the Monbusho) and a grant under the Monbusho International Science Research Program, American Israeli Bi-national Science Foundation, Direction des Sciences de la Matière du Commissariat à 1'Energie Atomique, France, Bundesministerium für Forschung und Technologie, FRG, A.P. Sloan Foundation and Junta Nacional de Investigação Científica e Tecnológica, Portugal.

\section{References}

[1] K. Ahmet et al., Nucl. Inst. Meth. A305 (1991) 275.

[2] R. Brun et al., GEANT3 User's Guide, CERN DD/EE/84-1.

[3] J. Allison et al., Comput. Phys. Commun. 47 (1987) 55.

[4] T. Sjöstrand, Comput. Phys. Commun. 39 (1986) 347;

T. Sjöstrand and M. Bengtsson, Comput. Phys. Commun. 43 (1987) 367.

[5] G. Marchesini and B.R. Webber, Nucl. Phys. B310 (1988) 461.

[6] Particle Data Group, Review of Particle Properties, Phys. Lett. 239B (1990) 1.

[7] R. Brun et al., ZEBRA User Guide, CERN DD/EE/85-6.

[8] M. Arignon et al., The Trigger System of the OPAL Experiment at LEP, CERN-PPE/9132, to be published in Nucl. Inst. Meth.

[9] OPAL Collaboration, M.Z. Akrawy et al., Z. Phys. C47 (1990) 505.

[10] S. Jadach et al., Z Physics at LEP1, CERN 89-08, ed. G. Altarelli et al., vol 1 (1989) 235; KORALZ, Version 3.7.

[11] M. Böhm, A. Denner and W. Hollik, Nucl. Phys. B304 (1988) 687;

F.A. Berends, R. Kleiss and W. Hollik, Nucl. Phys. B304 (1988) 712. 
[12] OPAL Collaboration, OPAL Silicon Microvertex Detector (Technical Proposal), CERN/LEPC 90-5.

[13] P.P. Allport et al., Nucl. Instr. and Meth. A310 (1991) 155.

[14] J.R. Carter et al., Nucl. Instr. and Meth. A286 (1990) 99;

A.A. Carter et al., Nucl. Instr. and Meth. A286 (1990) 107.

[15] P.v. Walter, G. Mildner; IEEE Trans. Nucl Sci. NS-32, 626 (1985).

[16] H.M. Fischer et al., Nucl. Instr. and Meth. A283 (1989) 492.

[17] H. Drumm et al., Nucl. Instr. and Meth. 176 (1980) 333.

[18] H.M. Fischer et al., Nucl. Instr. and Meth. A252 (1986) 331.

[19] H. Kreutzmann, Ph.D. Thesis, University Bonn, Bonn IR-91-08 (1991).

[20] D. Schaile et al., Nucl. Instr. and Meth. A242 (1986) 247.

[21] H. Breuker et al., Nucl. Instr. and Meth. A260 (1987) 329.

[22] M. Hauschild et al., CERN-PPE/91-130 (submitted to Nucl. Instr. and Meth.).

[23] O. Biebel, Diploma Thesis, University Bonn, Bonn IR-89-54 (1989).

[24] OPAL Collaboration, G.Alexander et al., Z. Phys. C52 (1991) 175.

[25] OPAL Collaboration, M.Z. Akrawy et al., Phys. Lett. 252B (1991) 290.

[26] OPAL Collaboration, M.Z. Akrawy et al., Zeit. Phys. C50 (1991) 373.

[27] An Ji-Gang et al., Nucl. Instr. and Meth. A267 (1988) 396.

[28] C. Beard et al., Nucl. Instr. and Meth. A286 (1990) 117.

[29] M. Z. Akrawy et al., Nucl. Instr. and Meth. A290 (1990) 76.

[30] S. Majewski, G. Charpak, A. Breskin, and G. Mikenberg, Nucl. Instr. and Meth. A217 (1983) 265.

[31] J. Allison et al, Nucl. Instr. and Meth. A310 (1991) 527.

[32] G.T.J. Arnison et al., Nucl. Instr. and Meth. A294 (1990) 431. 


\begin{tabular}{||r|rl||}
\hline Initialization & 70 & $\mathrm{sec}$ \\
\hline Kinematics & 1 & $\mathrm{sec} /$ event \\
\hline Tracking & 360 & $\mathrm{sec} /$ event \\
\hline Digitization & 18 & $\mathrm{sec} /$ event \\
\hline Reconstruction & 65 & $\mathrm{sec} /$ event \\
\hline
\end{tabular}

Table 1: Timing of various stages of the GOPAL simulation, for multihadronic $\mathrm{Z}^{0}$ decays. The timings are averages of runs on IBM3090 and VAX3600 machines, but have been scaled to corrrespond roughly to the standard CERN IBM/168 unit (roughly 4-5 mips).

\begin{tabular}{||||cr||c|c||}
\hline Variable & & Monte Carlo & Data \\
\hline Calorimeter Energy & $(\mathrm{GeV})$ & 3.38 & 3.42 \\
\hline Calorimeter $\theta$ & $(\mathrm{mrad})$ & 9.3 & 9.1 \\
\hline Calorimeter $\phi$ & $(\mathrm{deg})$ & 1.6 & 1.8 \\
\hline Tube chamber $\theta$ & $(\mathrm{mrad})$ & 6.6 & 7.3 \\
\hline Tube chamber $\phi$ & $(\mathrm{deg})$ & 1.4 & 1.6 \\
\hline
\end{tabular}

Table 2: Resolution of forward subdetectors, measured from the r.m.s. difference between left and right end quantities, for Bhabha scattering events.

\begin{tabular}{||c||r||r||}
\hline Category & Monte Carlo & Data \\
\hline$A C C$ & 1736 & 1736 \\
\hline$A C A C$ & 1570 & 1558 \\
\hline$C C$ & 2652 & 2460 \\
\hline$C \bar{A}$ & 916 & 905 \\
\hline$A \bar{C}$ & 10 & 6 \\
\hline
\end{tabular}

Table 3: Comparison of numbers of events with different combinations of $A$ and $C$ counters for the Fine Luminosity counters. 


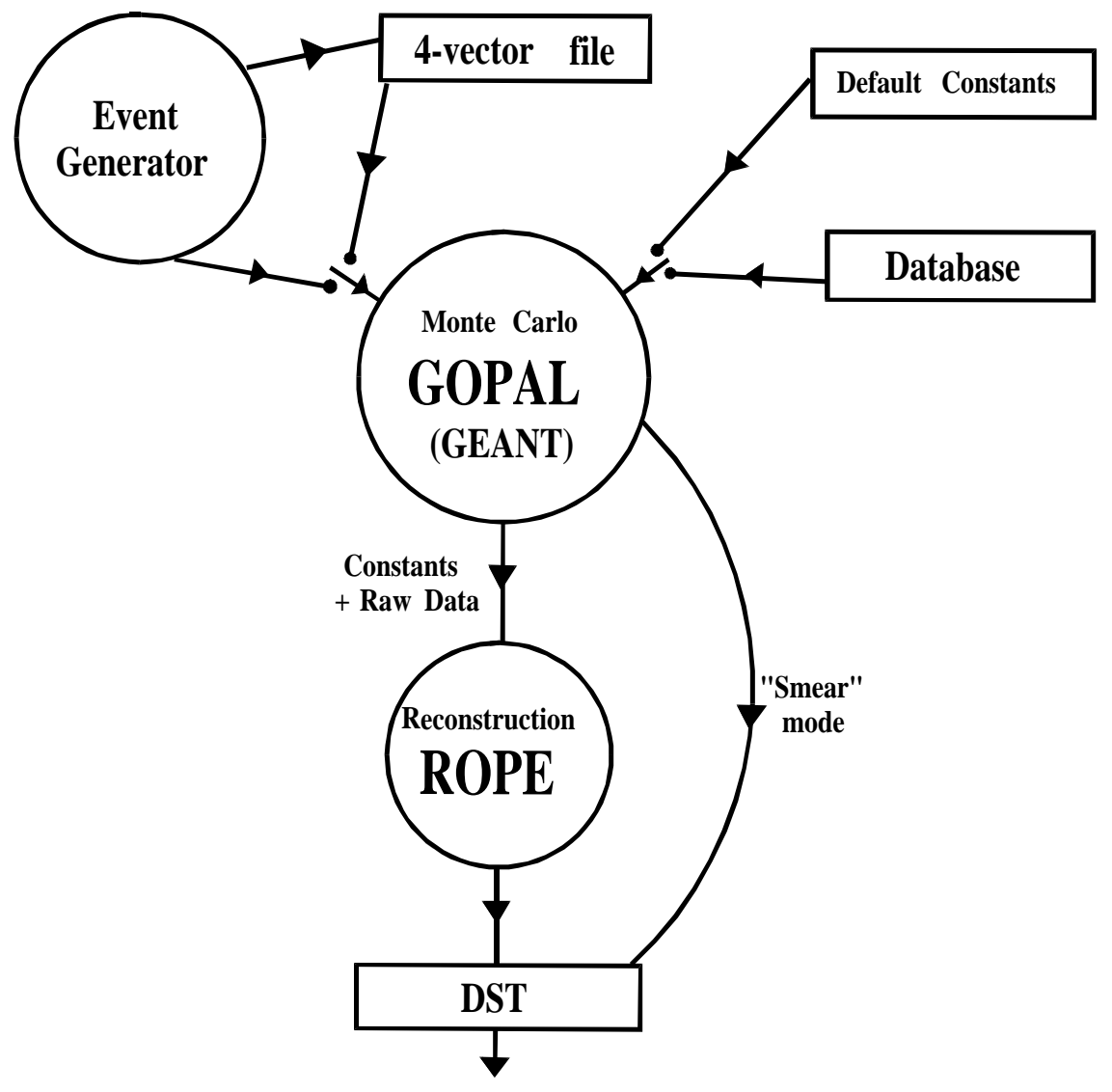

Physics Analysis

Figure 1: Outline of the organization of the GOPAL Monte Carlo program, and its relationship to the rest of the OPAL software. 


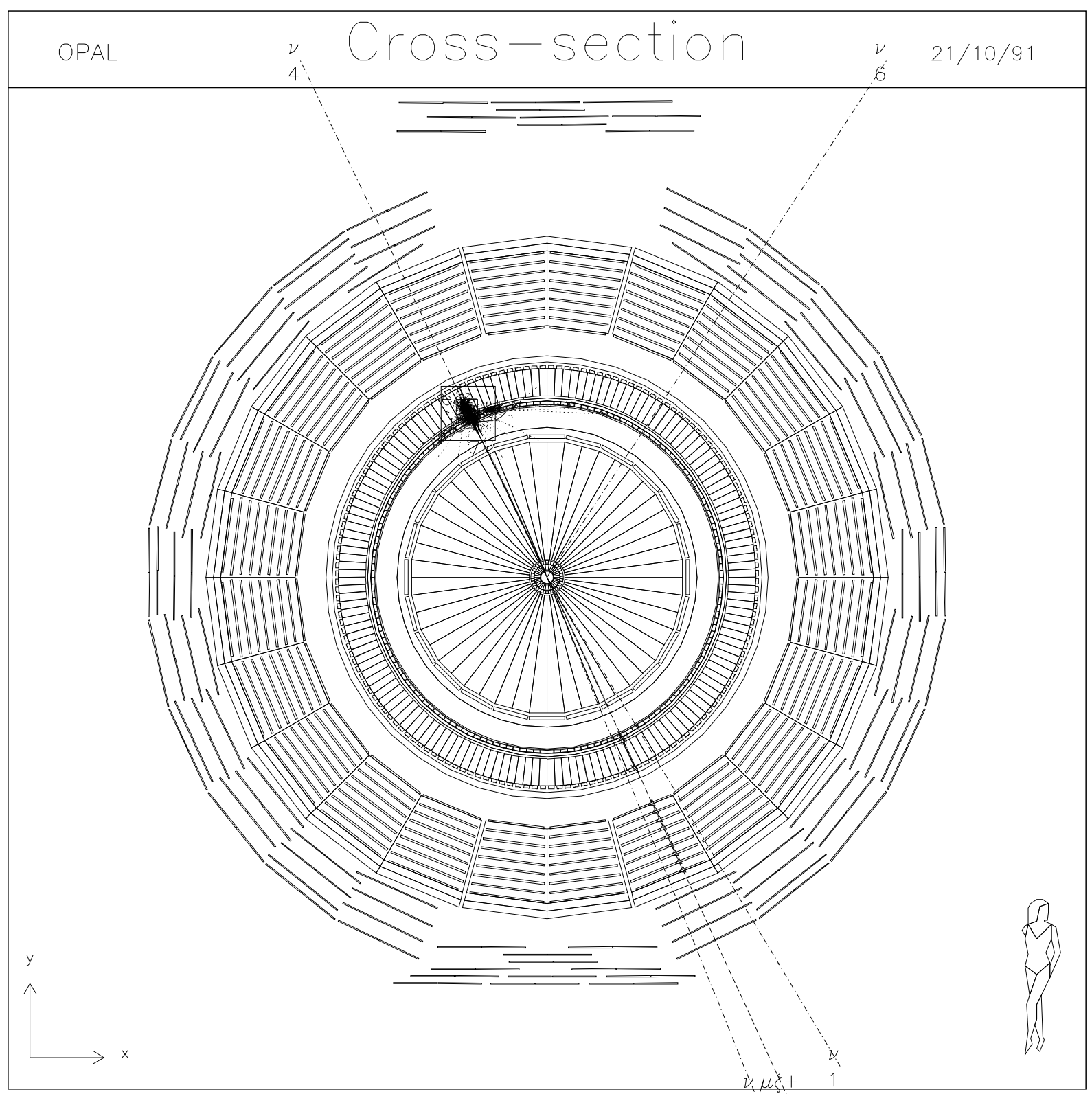

Figure 2: End view of the OPAL detector, as implemented in GOPAL. A simulated $\tau^{+} \tau^{-}$event is shown superimposed. For the sake of clarity the full detail of the geometry is not shown. 


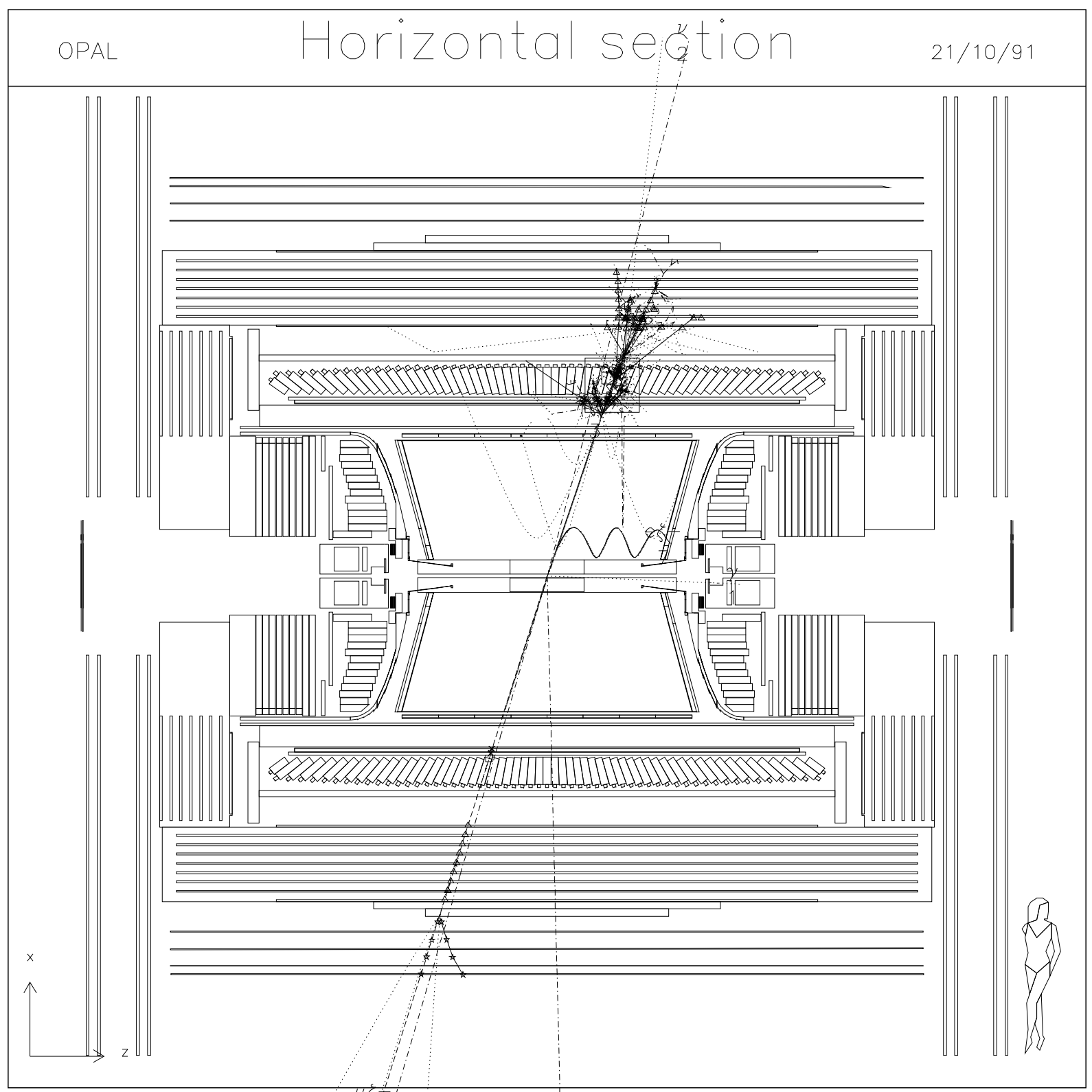

Figure 3: Top view of the OPAL detector, as implemented in GOPAL. A simulated $\tau^{+} \tau^{-}$event is shown superimposed. For the sake of clarity the full detail of the geometry is not shown. 

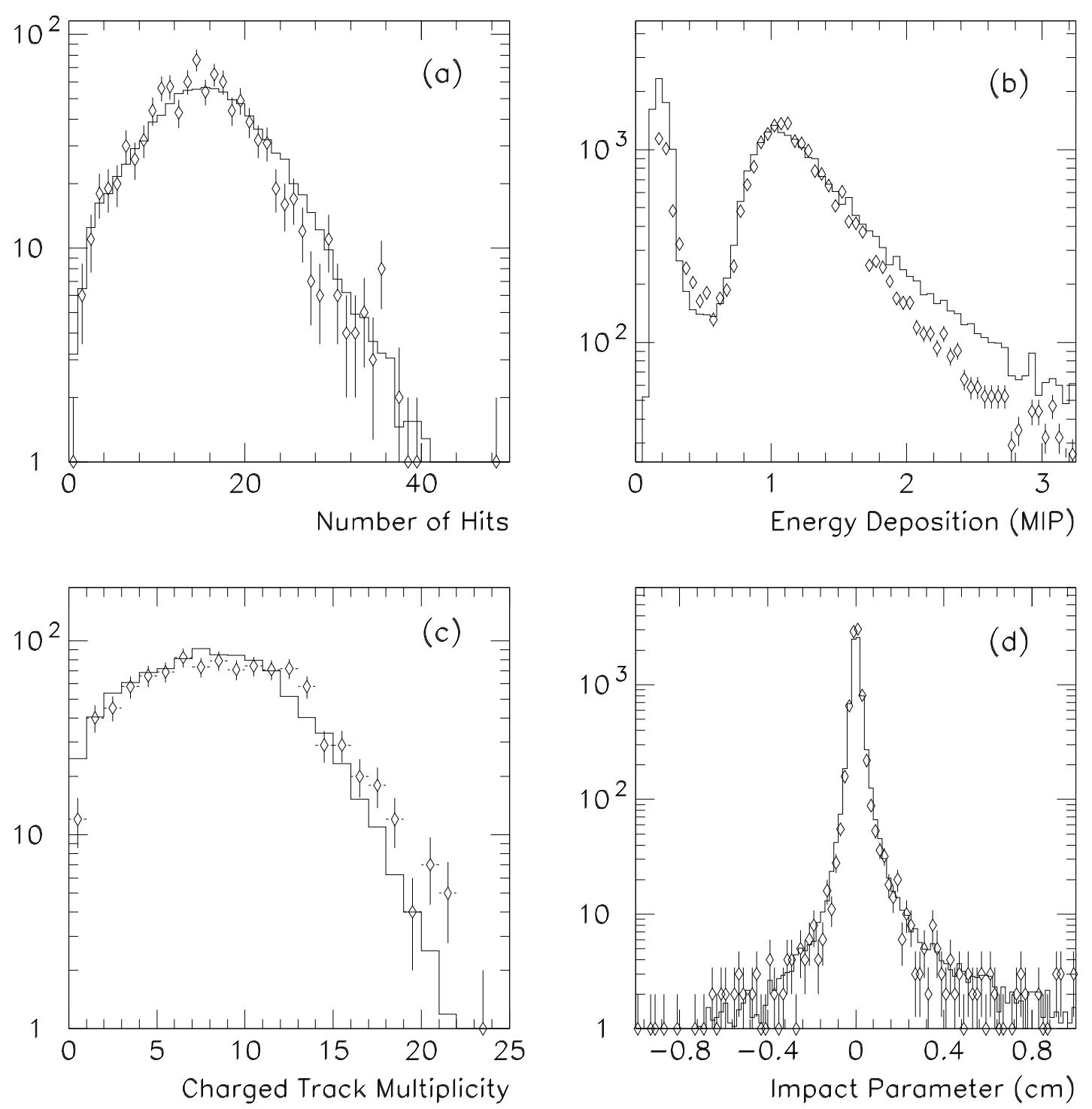

Figure 4: Distributions for the silicon microvertex detector in multihadronic events, shown for data (solid line) and Monte Carlo (points), normalized to equal numbers of events. The distributions shown are:

(a) The number of silicon hits per event in the inner silicon layer;

(b) The cluster pulse height distribution, which is a typical Landau distribution, and is scaled to be units of MIPs;

(c) The charged track multiplicity distribution for tracks with two associated silicon hits;

(d) The impact parameter distribution for tracks with two associated silicon hits. 

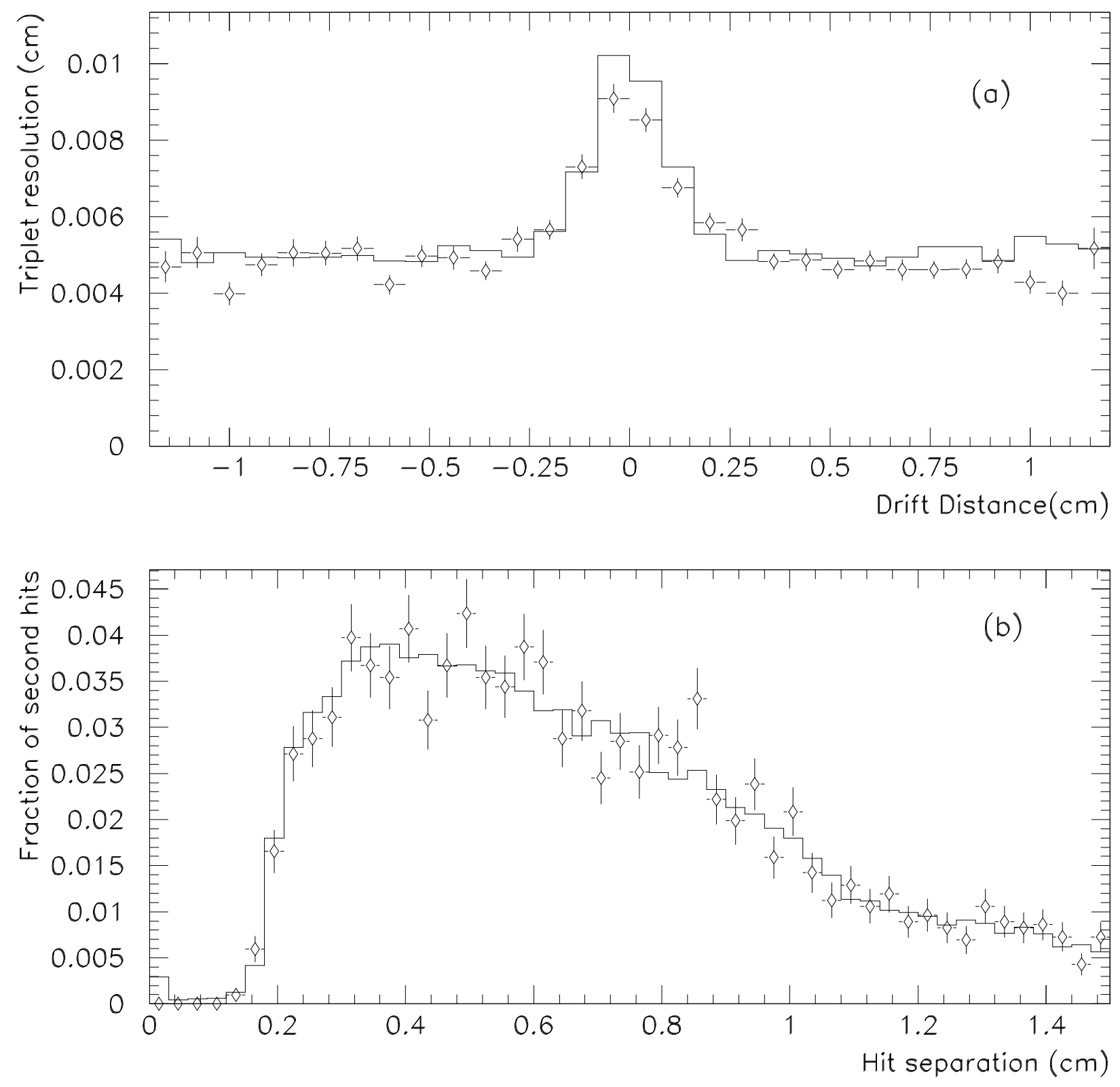

Figure 5: Comparison of real data (solid line) and Monte Carlo (points with error bars) for the vertex chamber:

(a) The triplet $r-\phi$ resolution of first hits in the vertex chamber axial cells;

(b) The difference in drift distance between axial 'second' hits and the previous hit on the same wire. 

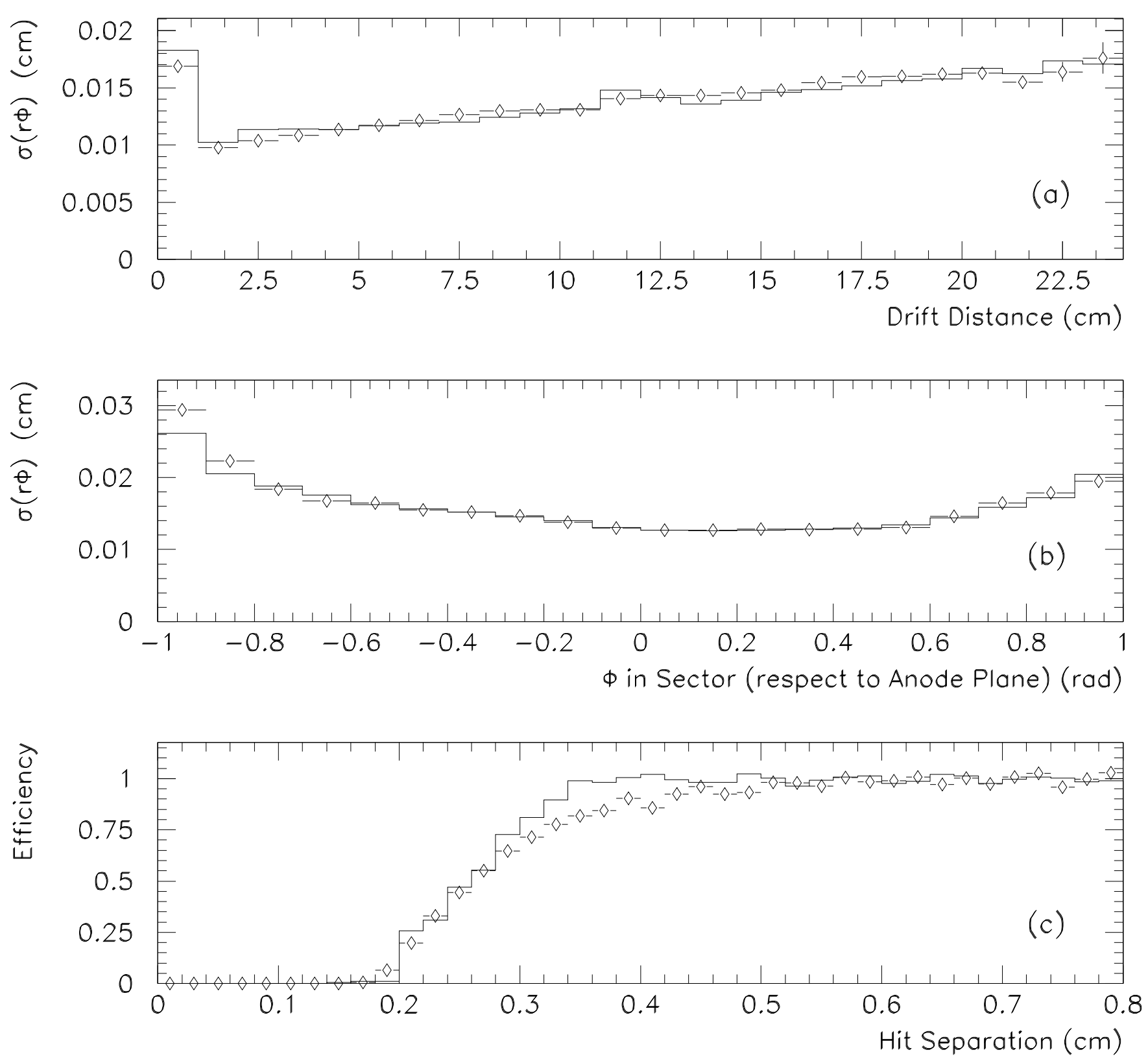

Figure 6: Comparison of real data (solid line) and Monte Carlo (points with error bars) for the jet chamber:

(a) Space point resolution $\sigma_{r \phi}$ as a function of the drift distance from the signal wires;

(b) $\sigma_{r \phi}$ as a function of the local track angle $\Phi_{\text {local }}$ with respect to the sense wire plane;

(c) Efficiency to separate two hits as a function of the hit separation. 

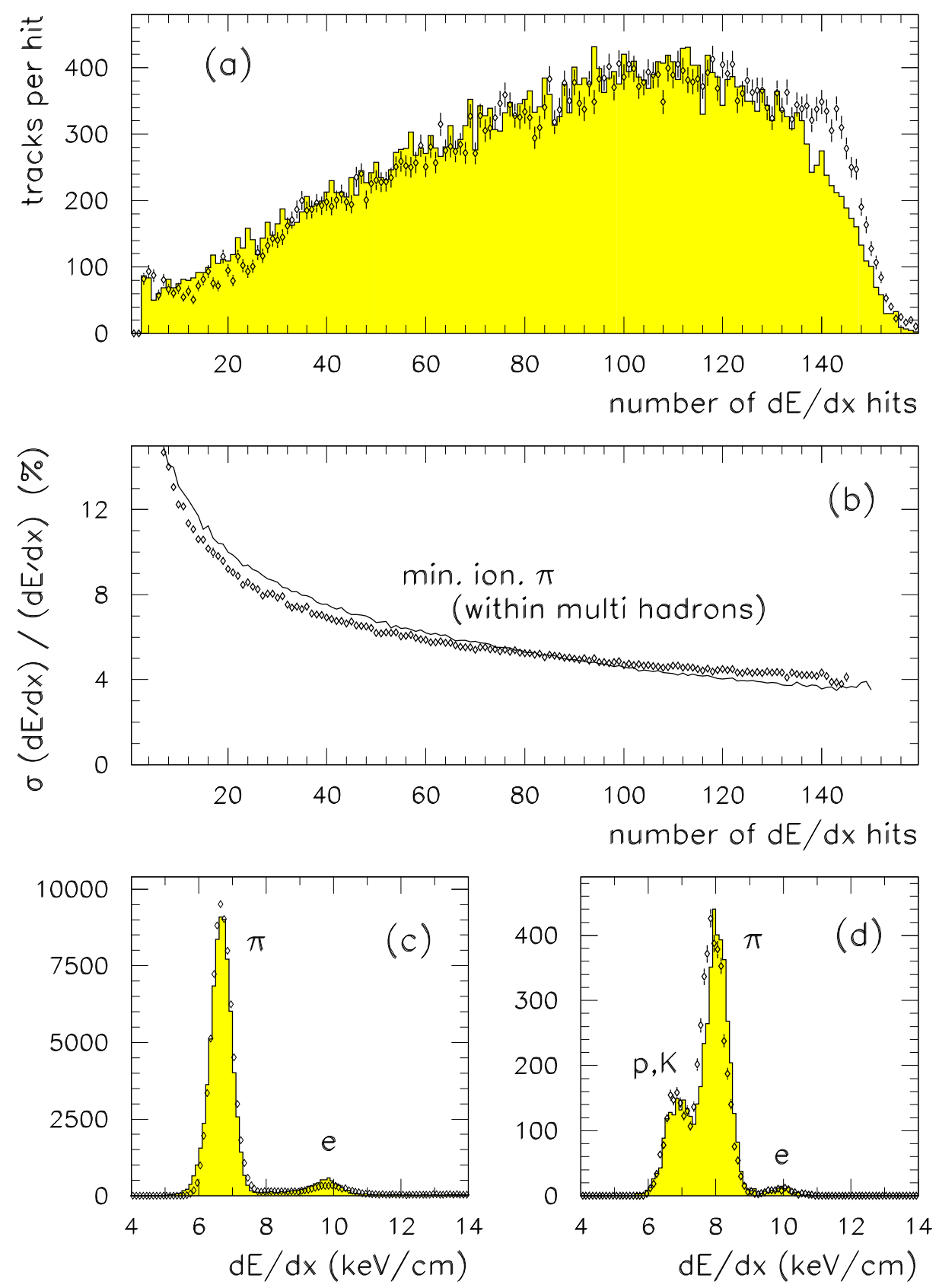

Figure 7: Comparison of real data (solid line) and Monte Carlo (points with error bars) for the jet chamber. The histograms $(a, c, d)$ are normalized to equal numbers of events.

(a) Distribution of the number of hits per track in multihadronic events useable for the calculation of the truncated mean for tracks in the polar angle region $|\cos \theta|<0.7$. At least 40 hits are obtained for $89 \%$ of the tracks;

(b) Relative resolution $\frac{\sigma_{d E / d x}}{d E / d x}$ as a function of the number of hits in multihadronic events used for the calculation of the truncated mean for tracks in the polar angle region $|\cos \theta|<0.7$;

(c,d) Truncated mean for tracks in multihadronic events in the momentum ranges $0.4-0.8$ $\mathrm{GeV} / c$ and $2.5-4.0 \mathrm{GeV} / c$ respectively. 

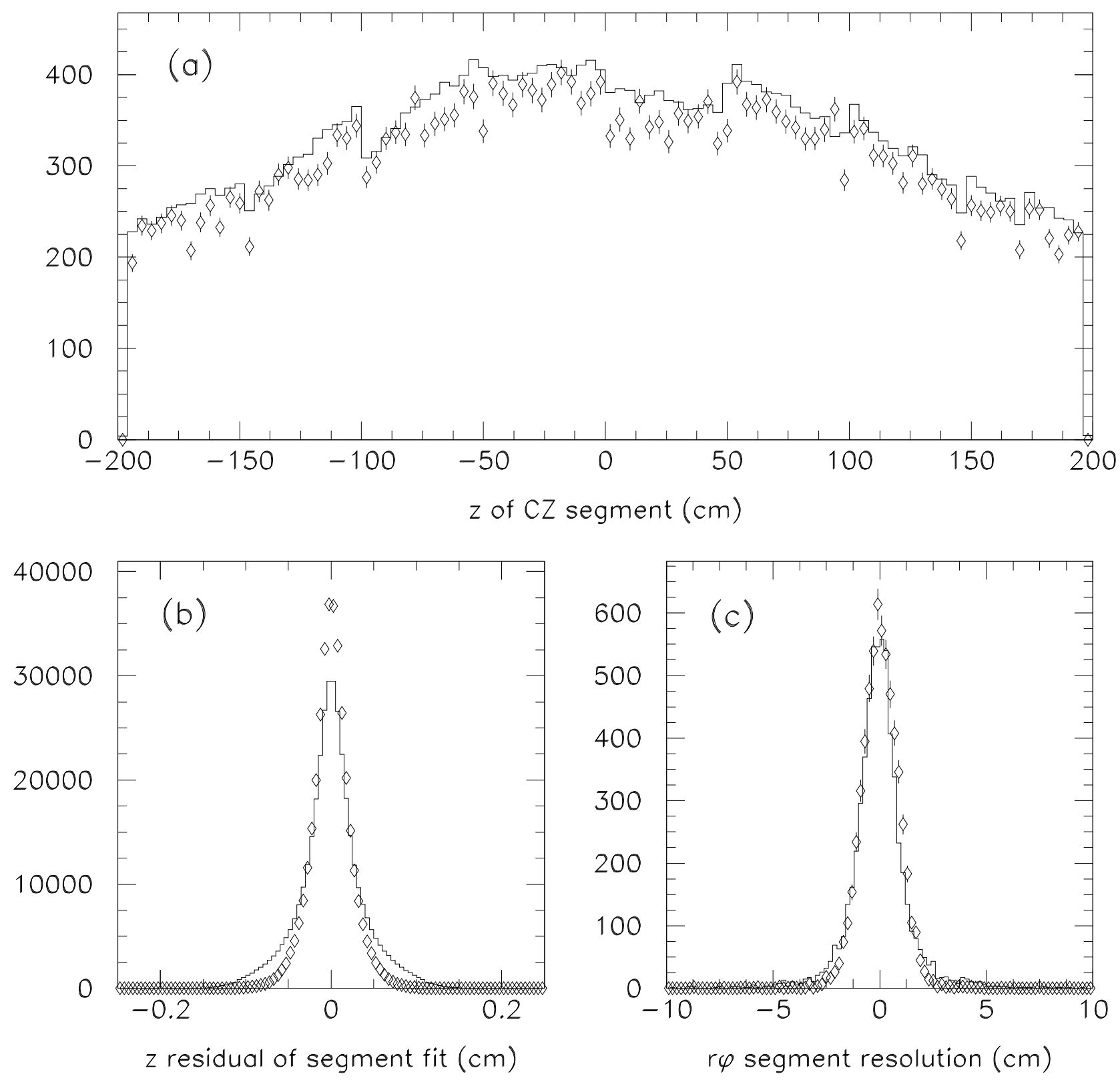

Figure 8: Comparison between data (solid line) and Monte Carlo (points) for the Z-chambers. The histograms are normalized to equal numbers of events.

(a) Number of reconstructed segments, as a function of $z$;

(b) Residual in $z$ of the line fit to the segments;

(c) Difference at the centre of the Z-chambers between the $r-\phi$ coordinate of the segment and the extrapolation of the track in the jet chamber (accuracy of a few hundred $\mu \mathrm{m}$ ). 

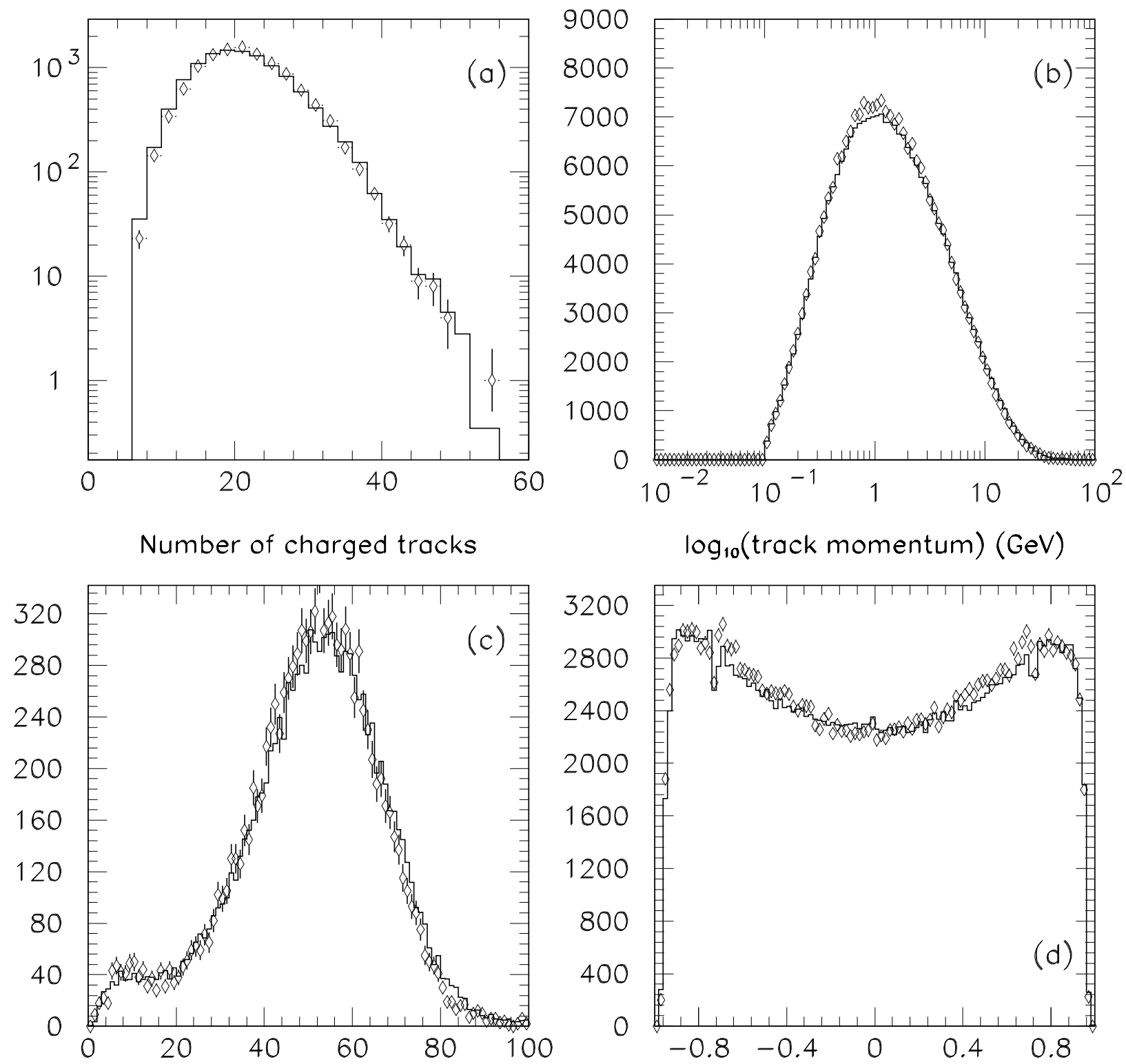

Sum of track momenta $(\mathrm{GeV})$

$$
\cos (\vartheta)
$$

Figure 9: Comparisons between data (solid line) and Monte Carlo (points with errors) for multihadronic events. In all cases the plots are normalized to equal numbers of events.

(a) The number of "good tracks" per event;

(b) The distribution of individual track momenta;

(c) The total momentum summed over all "good" tracks;

(d) The cosine of the polar angle. 

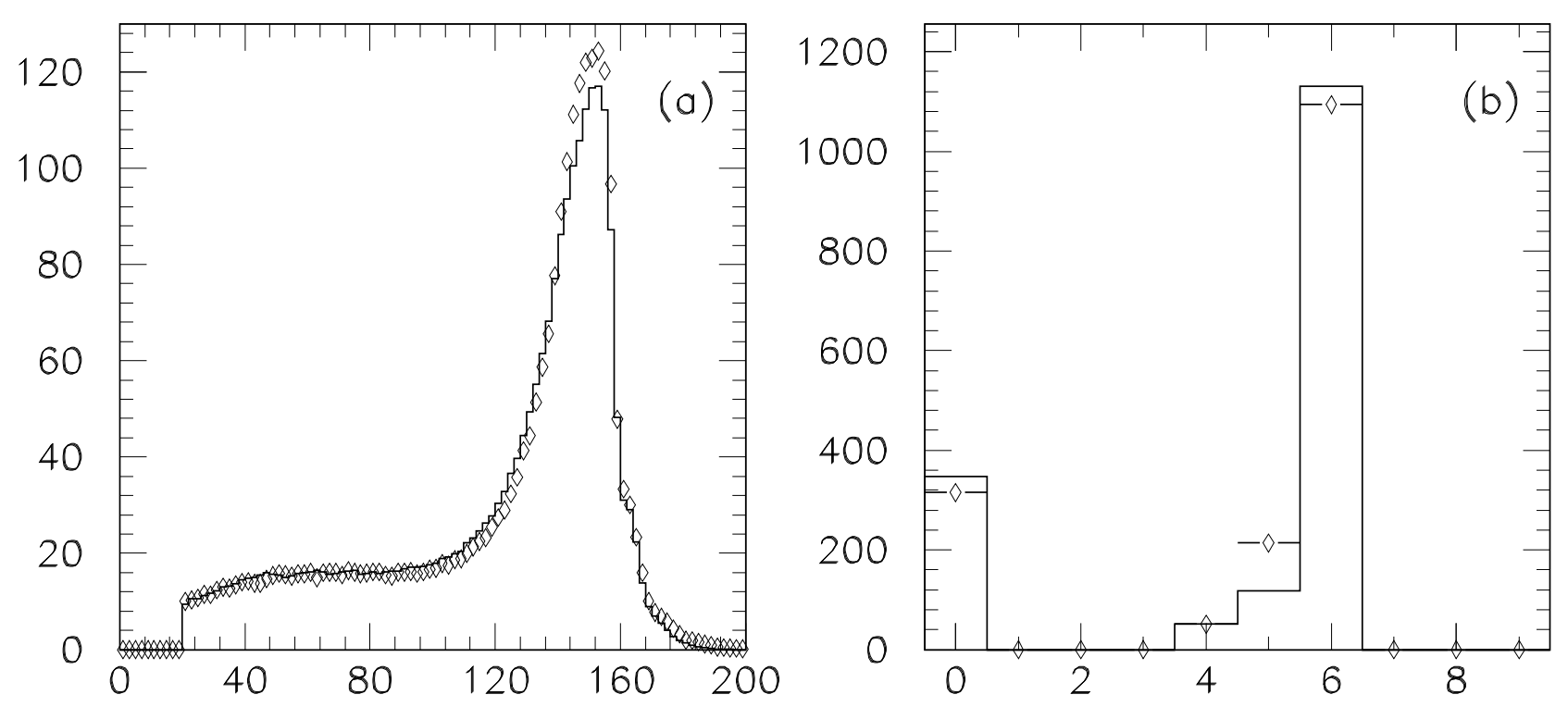

Number of $\mathrm{CJ}$ hits

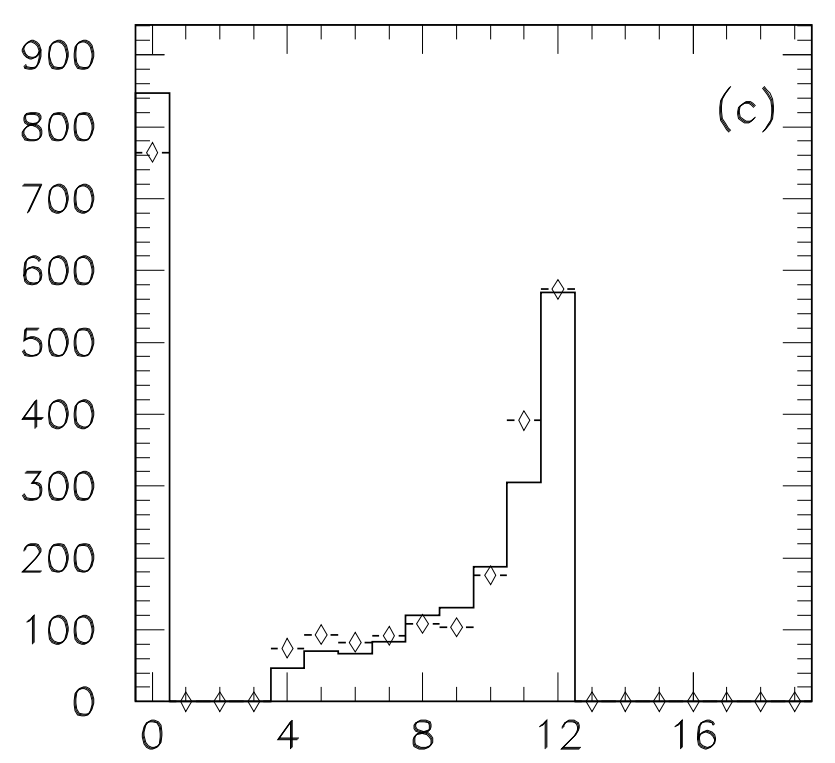

Number of CV axial hits

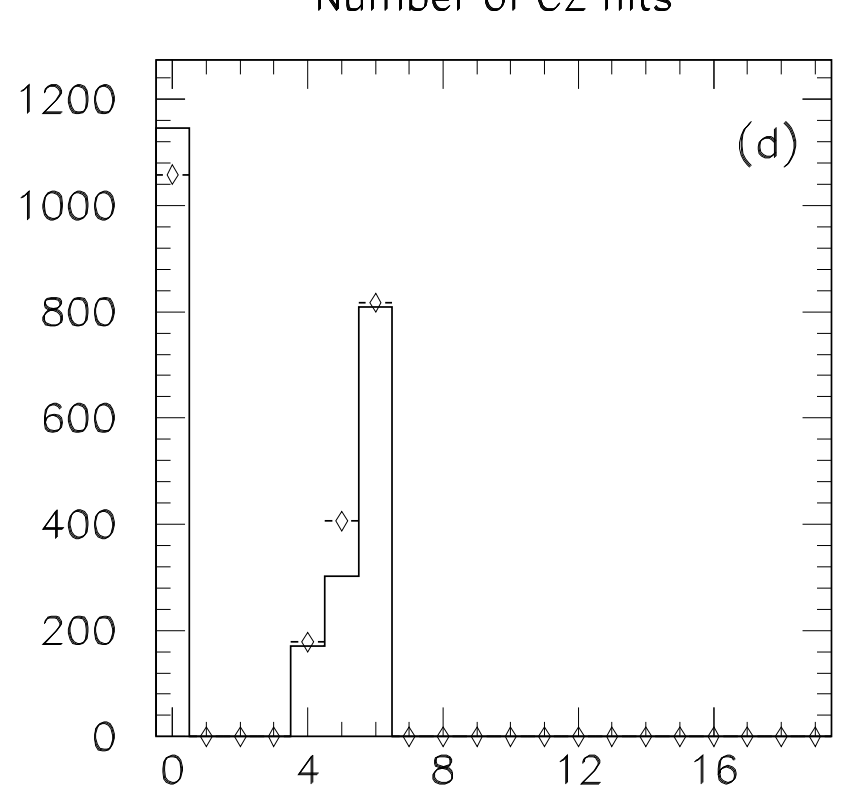

Number of CV stereo hits

Figure 10: Comparisons between data (solid line) and Monte Carlo (points with errors) for multihadronic events. In all cases the plots are normalized to equal numbers of events.

(a) The number of CJ hits on a track;

(b) The number of $\mathrm{CZ}$ hits on a track (restricted to $|\cos \theta|<0.68$ );

(c) The number of $\mathrm{CV}$ axial hits on a track;

(d) The number of CV stereo hits on a track. 


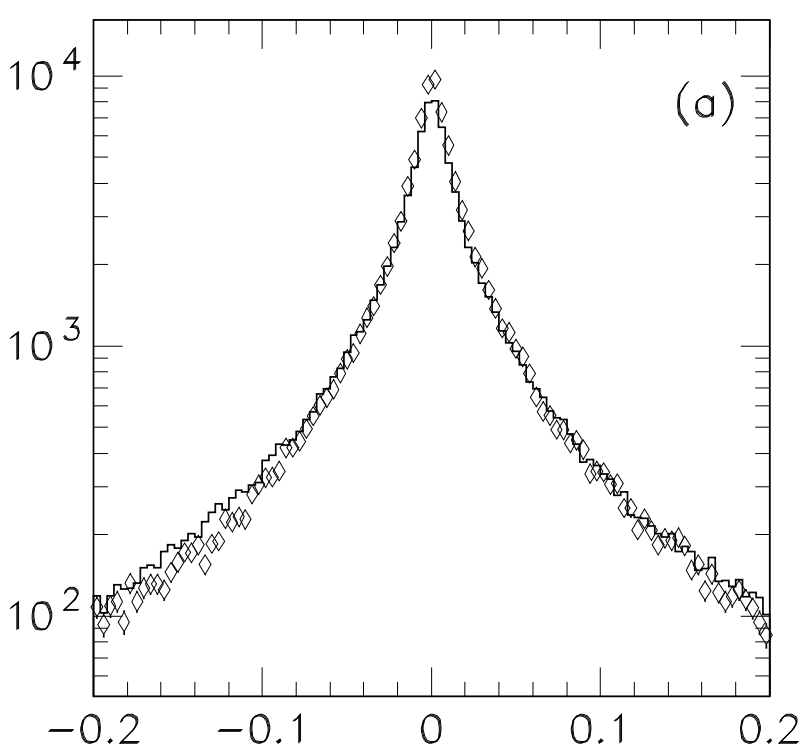

Transverse Impact Parameter $d_{0}(\mathrm{~cm})$

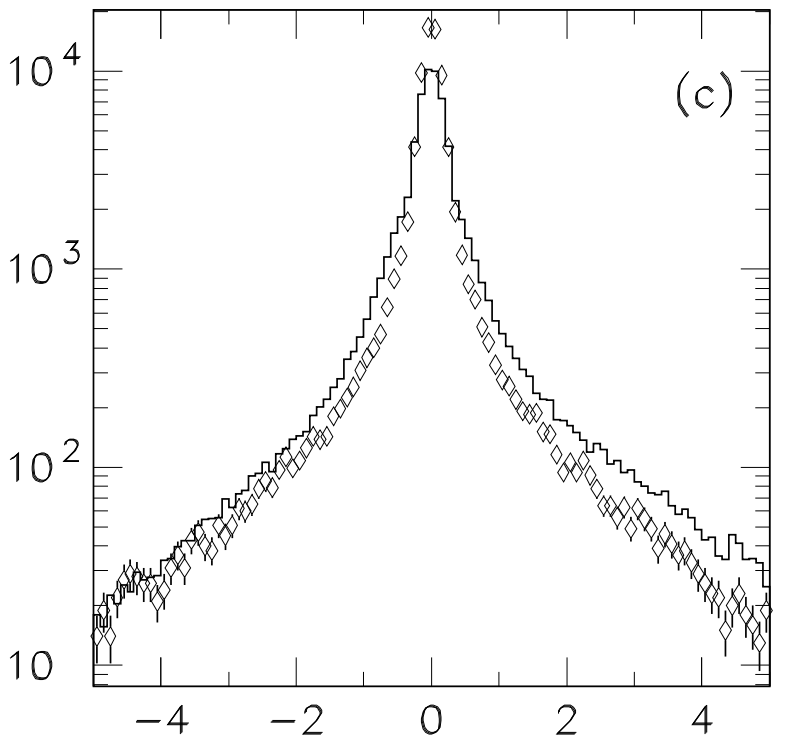

Longitudinal Impact Parameter $z_{0}(\mathrm{~cm})$

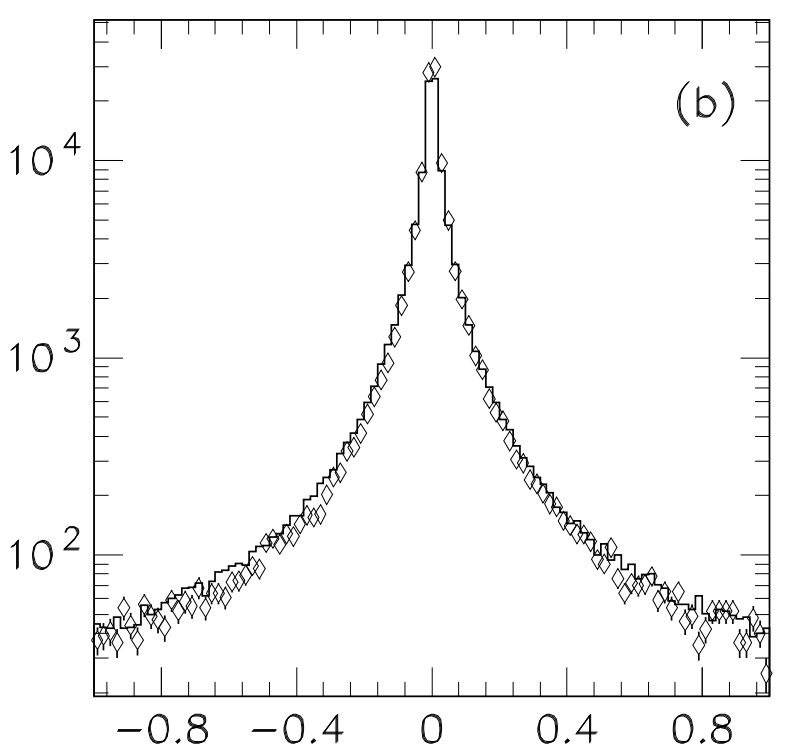

Transverse Impact Parameter $d_{0}(\mathrm{~cm})$

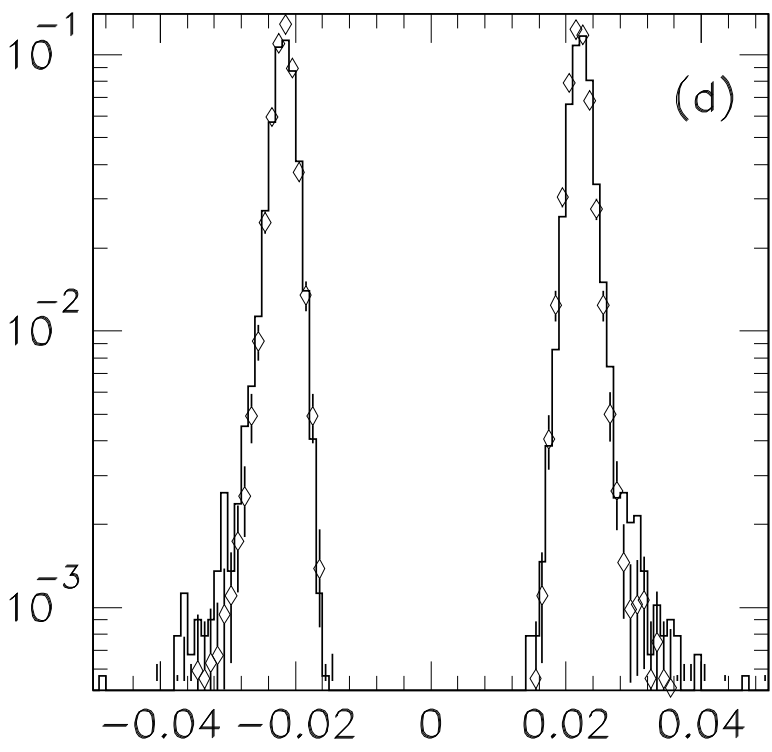

$1 / \mathrm{p}\left(\mathrm{GeV}^{-1}\right)$ in $\mu^{+} \mu^{-}$events

Figure 11: Comparisons between data (solid line) and Monte Carlo (points with errors) for multihadronic events (a-c) or $\mu^{+} \mu^{-}$events (d). In all cases the plots are normalized to equal numbers of events.

(a) The impact parameter in the $r-\phi$ plane;

(b) The impact parameter in the $r-\phi$ plane;

(c) The impact parameter in $z$;

(d) The distribution of $1 / p$ (signed by charge) for tracks in $\mu^{+} \mu^{-}$events. 

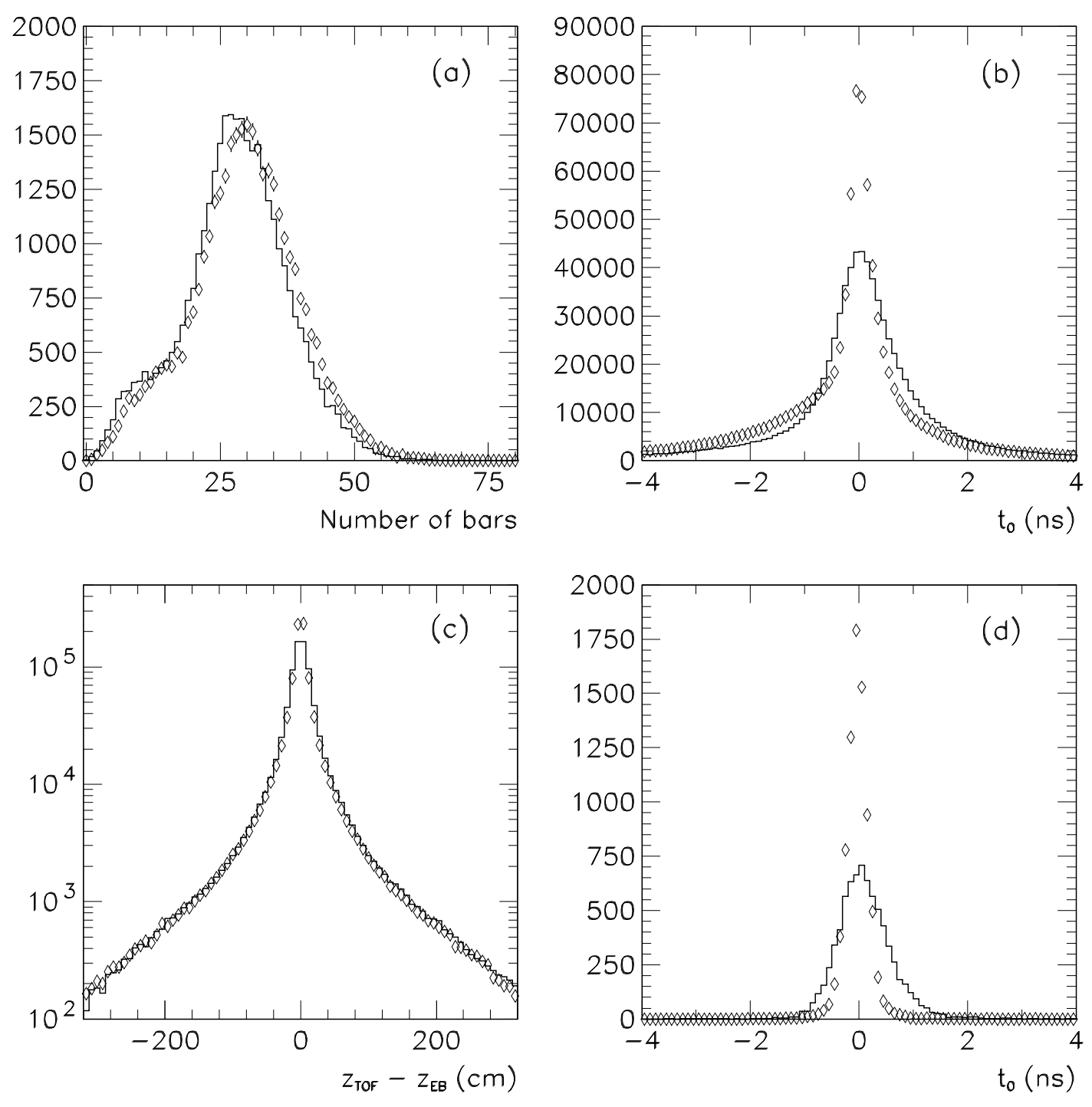

Figure 12: Comparison of real data (solid line) and Monte Carlo (points with error bars) for the Time-of-Flight system: In all cases the plots are normalized to equal numbers of events.

(a) Number of TOF bars with both left and right discriminators firing in multihadronic events;

(b) Time measured - time expected for a photon for TOF bars with both discriminators fired in multihadronic events;

(c) $z$ measured by TOF (from time difference) $-z$ measured by the barrel lead glass for TOF bars in multihadronic events (logarithmic scale);

(b) Same as (b) but for $\mathrm{Z}^{0}$ decays to $\mu^{+} \mu^{-}$, where the muons are highly relativistic. 


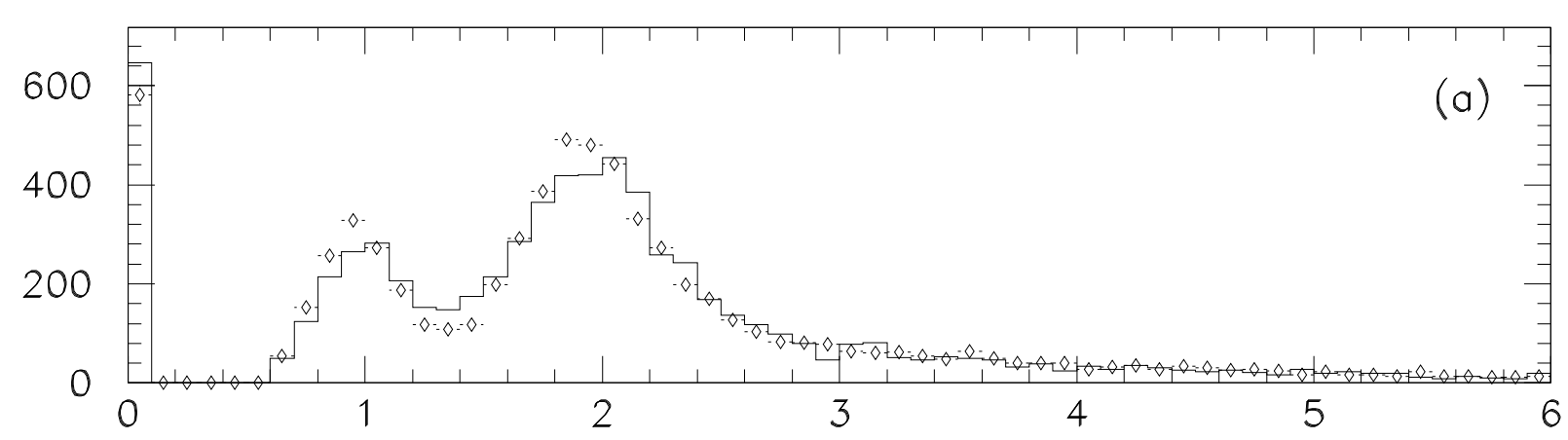

Multiplicity
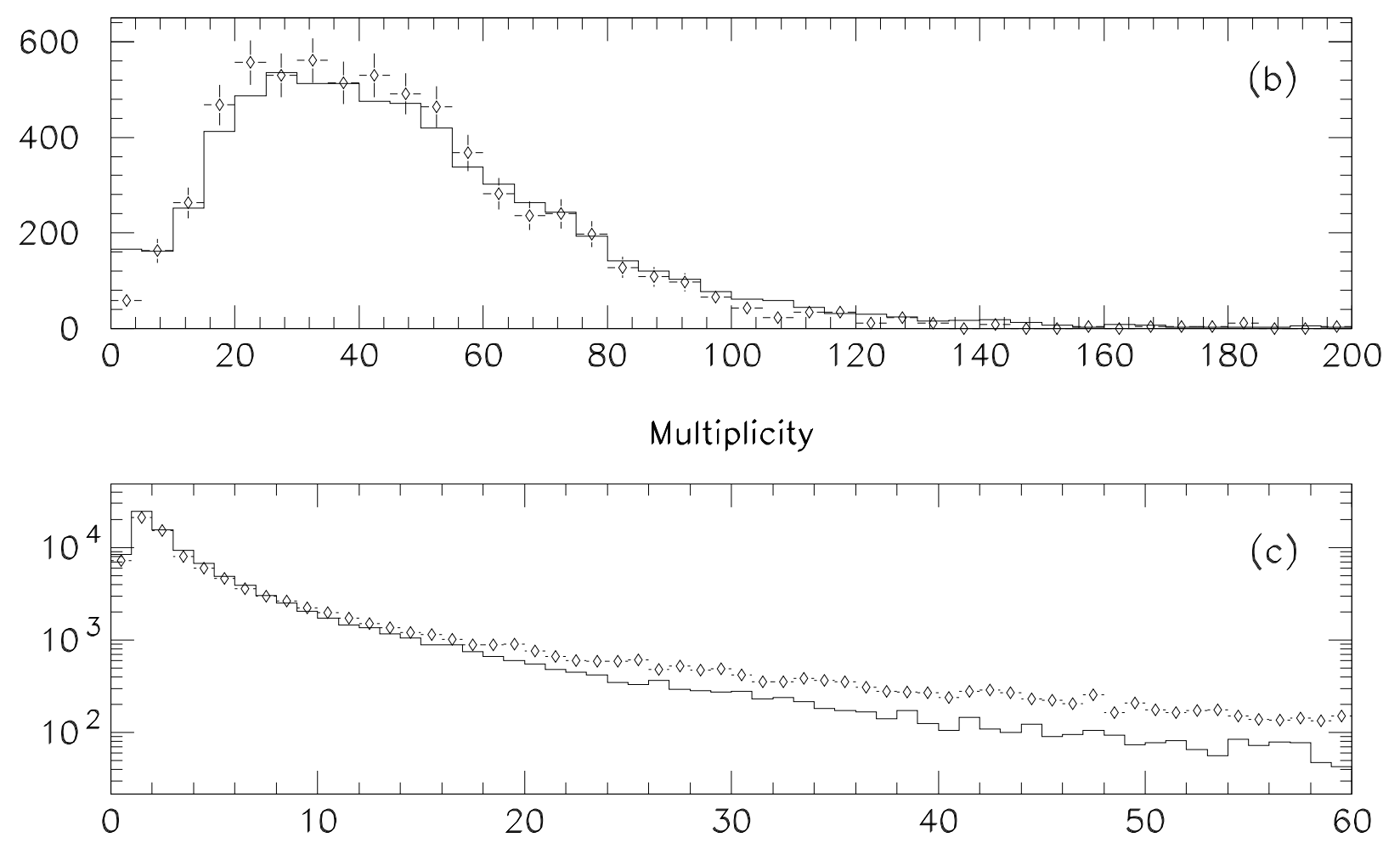

Multiplicity

Figure 13: Comparison of data (solid line) and Monte Carlo (points with errors) for the Presampler Barrel. In all cases the plots are normalized to equal numbers of events.

(a) Multiplicity associated to tracks in $\mu^{+} \mu^{-}$events;

(b) Multiplicity associated to tracks in Bhabha events;

(c) Multiplicity associated to tracks in multihadron events; note the logarithmic scale. 

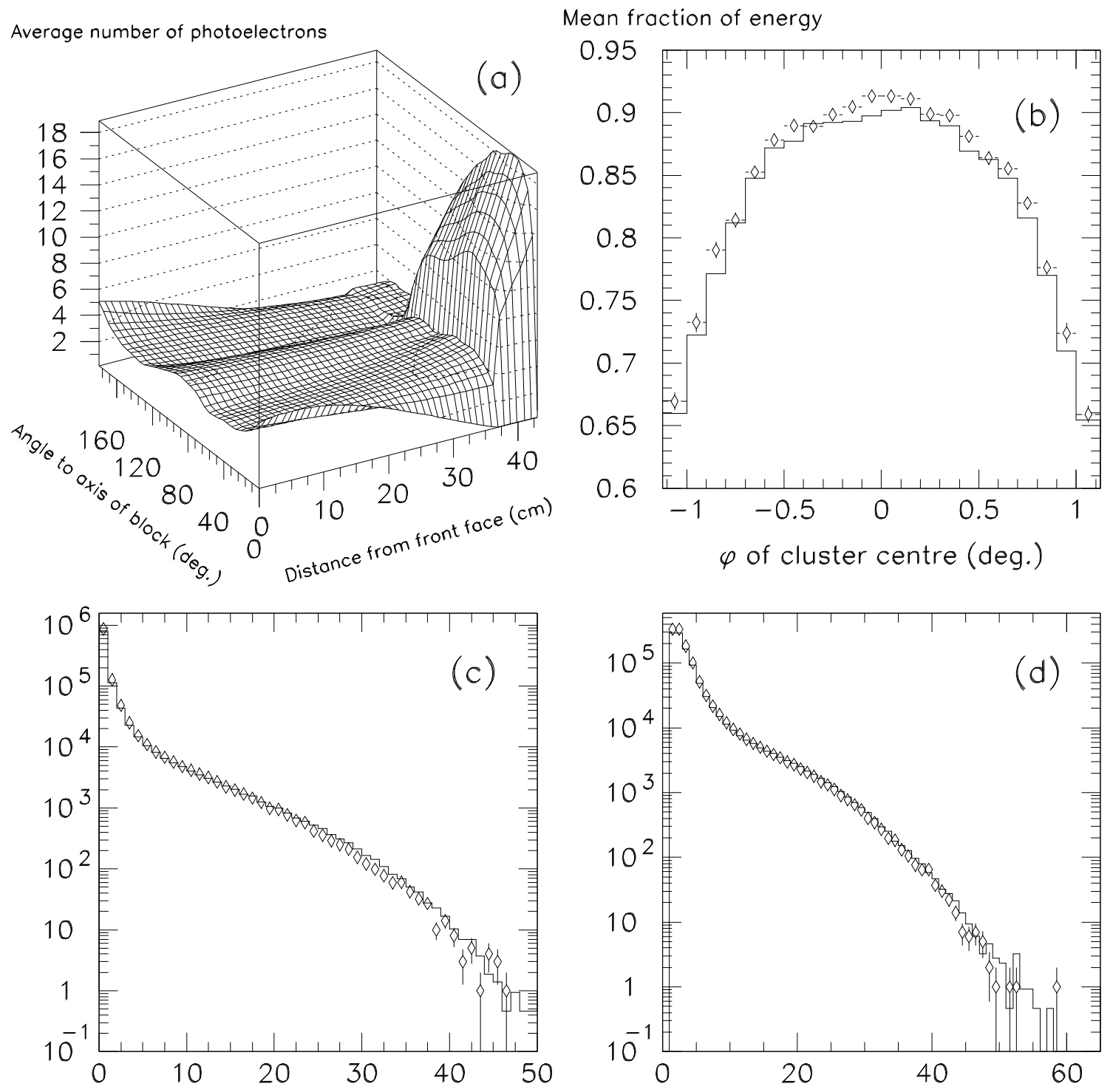

Energy of a cluster (GeV)

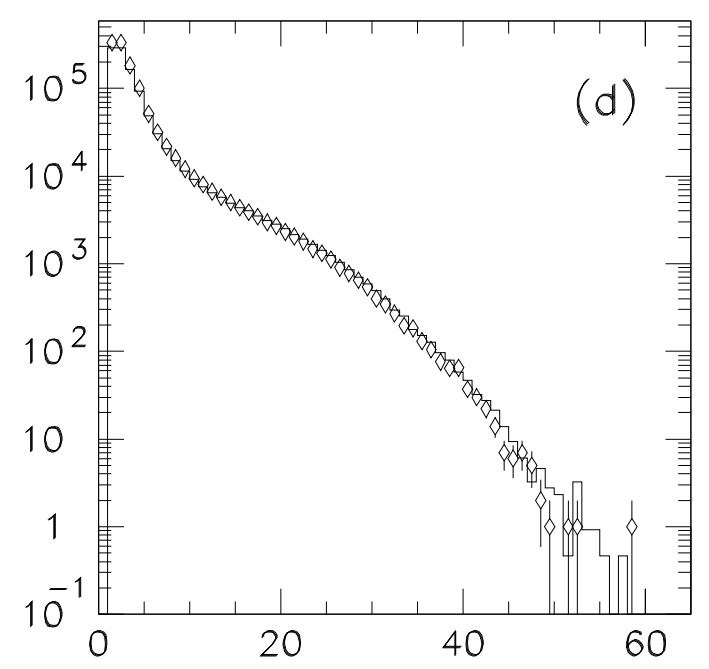

Number of blocks in a cluster

Figure 14: Plots relating to the Electromagnetic Barrel Calorimeter.

(a) Average number of photo-electrons detected per $\mathrm{cm}$ of track length for highly relativistic particles in a barrel lead glass block;

(b)-(d) Comparison of Monte Carlo (points with errors) with data (solid line) The histograms $(c, d)$ are normalized to equal numbers of events.

(b) Mean fraction of energy in the most energetic block for high energy electron clusters in barrel Bhabha events as a function of the azimuthal angle of the cluster centre measured from the middle of the block;

(c) Energy of barrel electromagnetic clusters in multihadron events;

(d) Number of blocks in barrel electromagnetic clusters in multihadron events. 


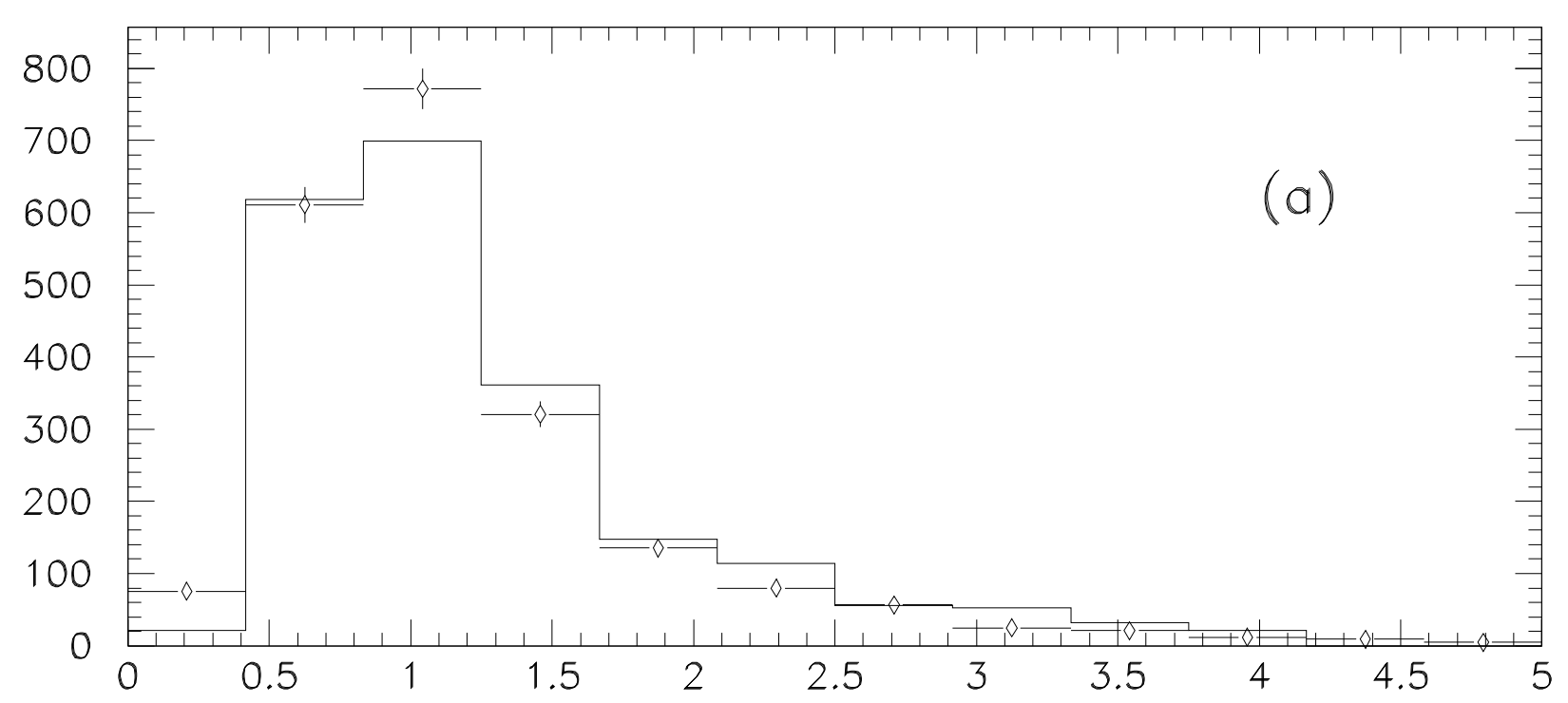

PE multiplicity

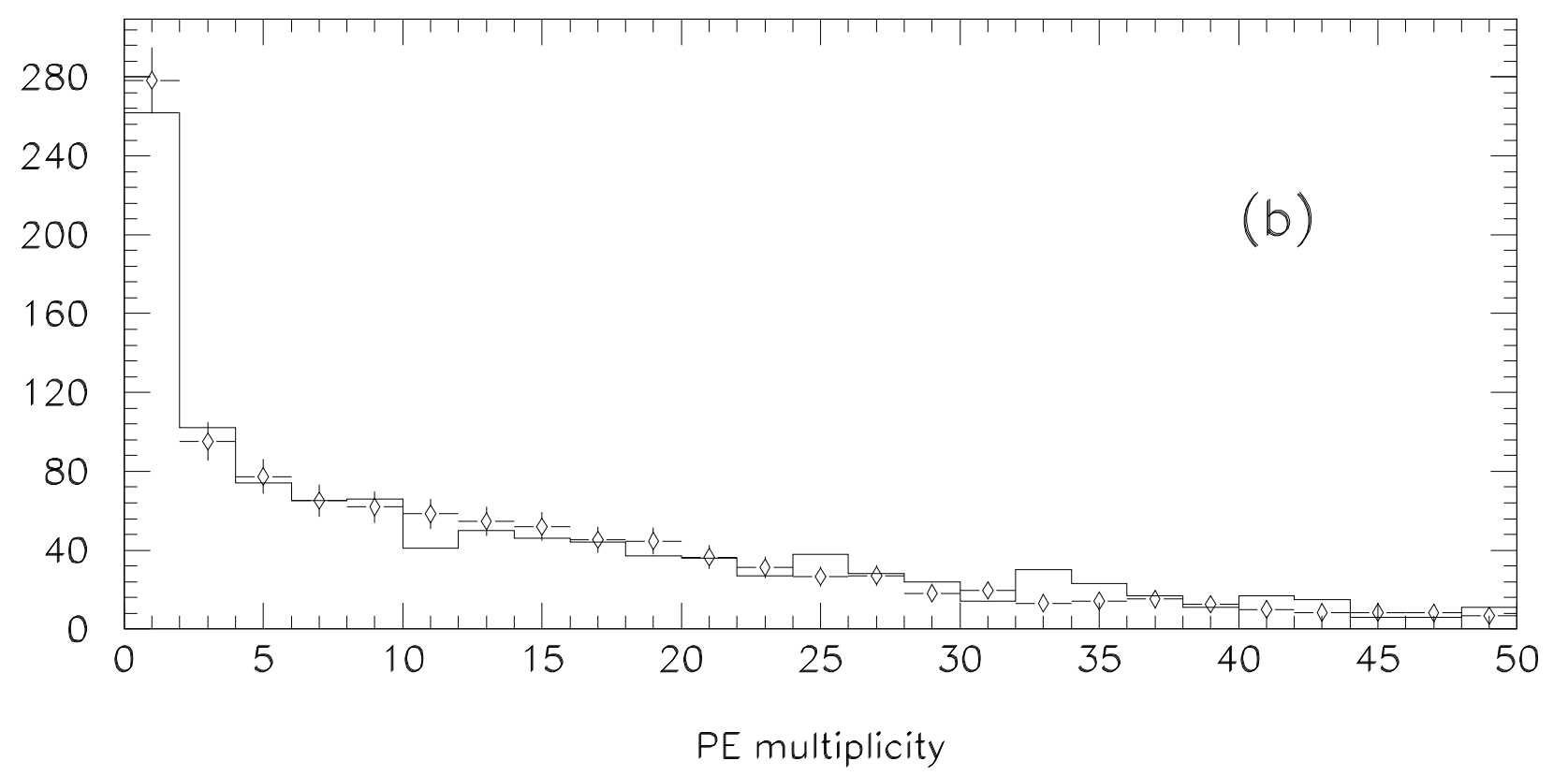

Figure 15: Comparison of data (solid line) and Monte Carlo (points with errors) for the Presampler Endcap. The histograms are normalized to equal numbers of events.

(a) Multiplicity associated to $\mu^{+} \mu^{-}$tracks;

(b) Multiplicity associated to tracks in single-track decays of $\tau$-leptons. 

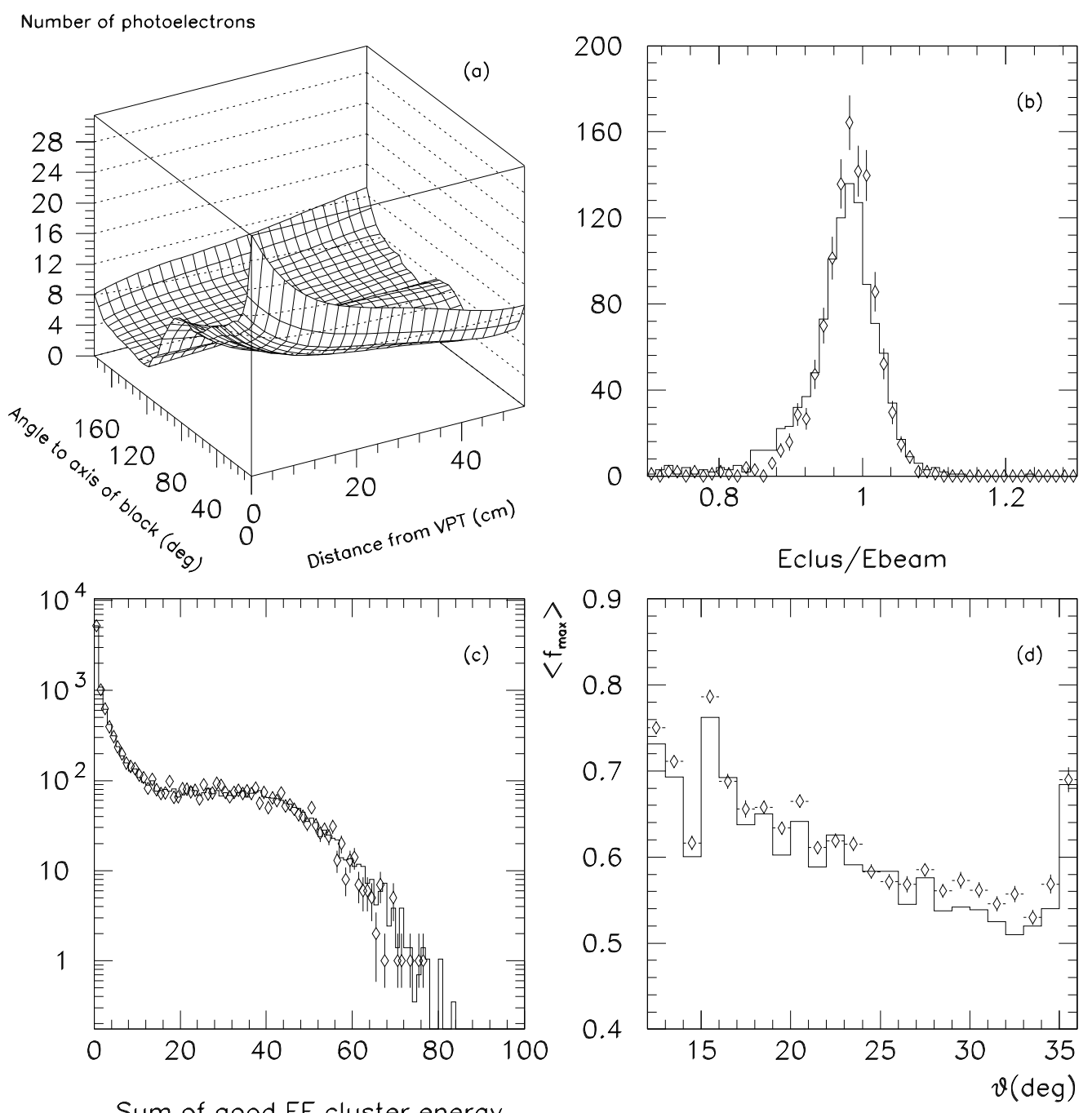

Sum of good EE cluster energy

Figure 16: Plots relating to the Electromagnetic Endcap Calorimeter.

(a) Average number of photoelectrons detected per $\mathrm{cm}$ of track length for highly relativistic particles in an endcap lead glass block;

(b)-(d) Comparison of Monte Carlo (points with errors) with data (solid line). The histograms $(b, c)$ are normalized to equal numbers of events.

(b) Cluster energy divided by beam energy for the two highest energy clusters in Bhabha events in the region $0.85<|\cos \theta|<0.9$;

(c) Total energy in endcap electromagnetic clusters in multihadron events;

(d) Mean fraction of energy in the block with most energy in the two highest energy clusters in Bhabha events as a function of $\theta$. 


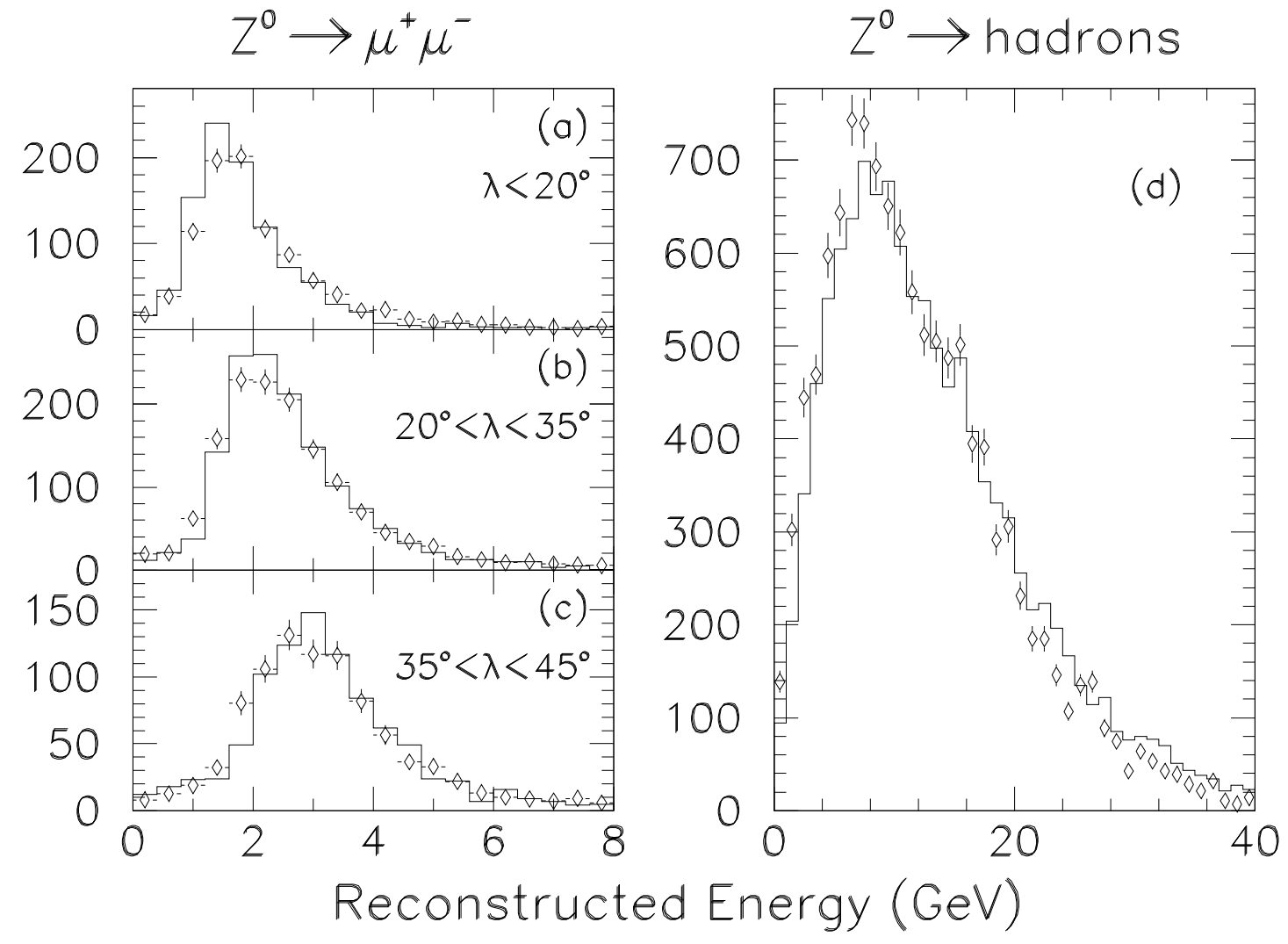

Figure 17: Comparison of data (solid line) and Monte Carlo (points with errors) for the Hadron Barrel and Endcap calorimeters. In all cases the plots are normalized to equal numbers of events.

(a)-(c) The reconstructed hadronic energy for penetrating muons for different angles of incidence, $\lambda$, on the hadron calorimeter barrel and endcap.

(d) The total observed hadronic energy for multihadronic $Z^{0}$ decays. 

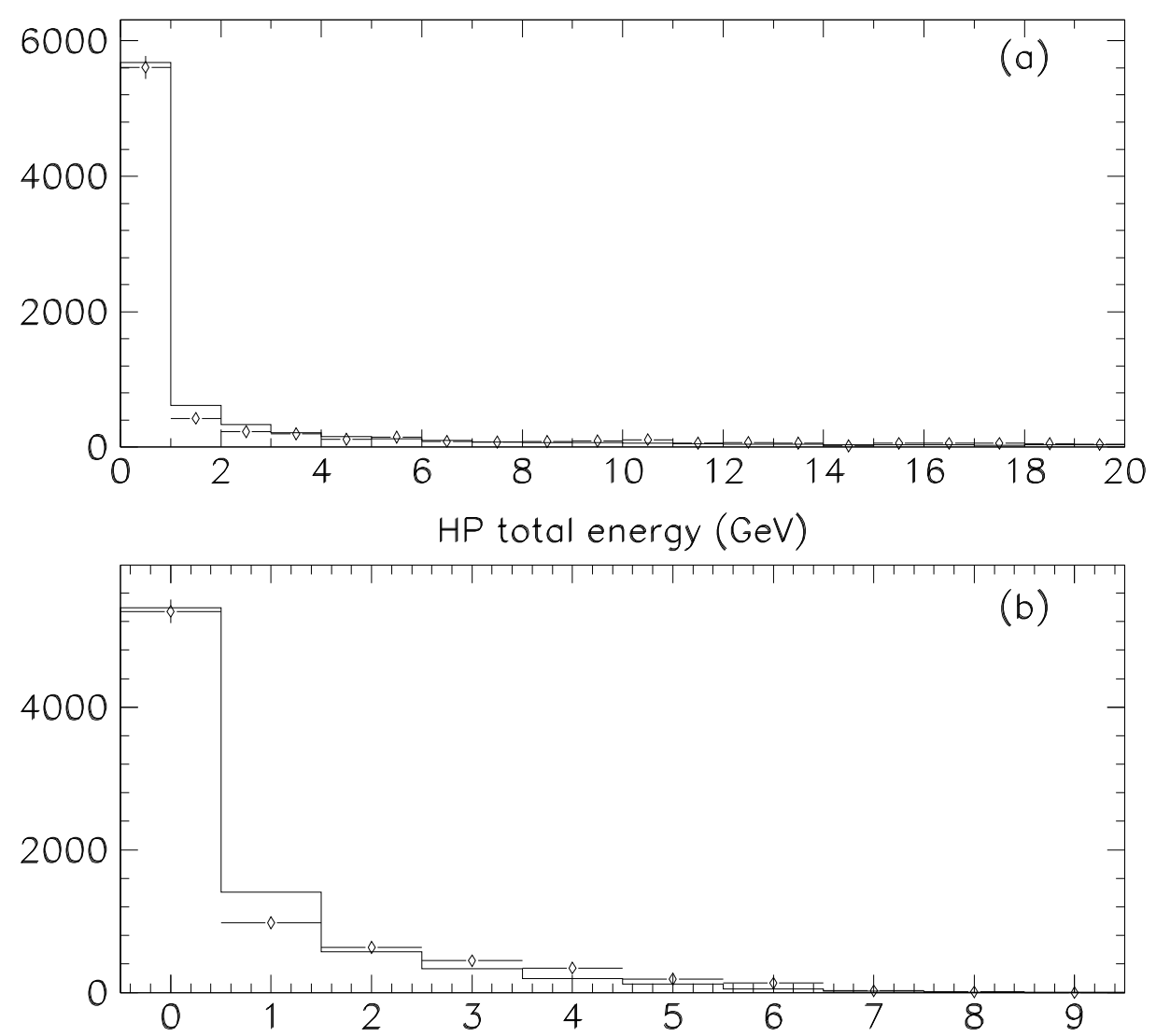

HP number of clusters

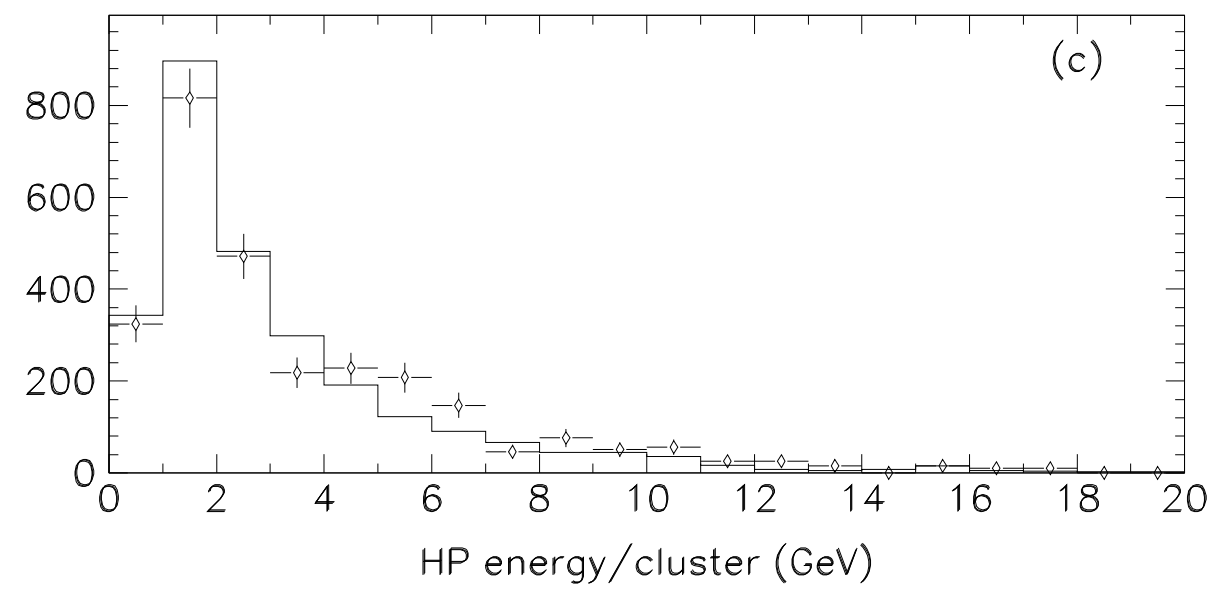

Figure 18: Comparison between GOPAL and data for the hadron pole tip calorimeter. The solid lines represent the data and the points represent the simulation. In all cases the plots are normalized to equal numbers of events.
(a) Total energy in the hadron pole tip;
(b) Number of clusters per event;
(c) Energy per cluster. 

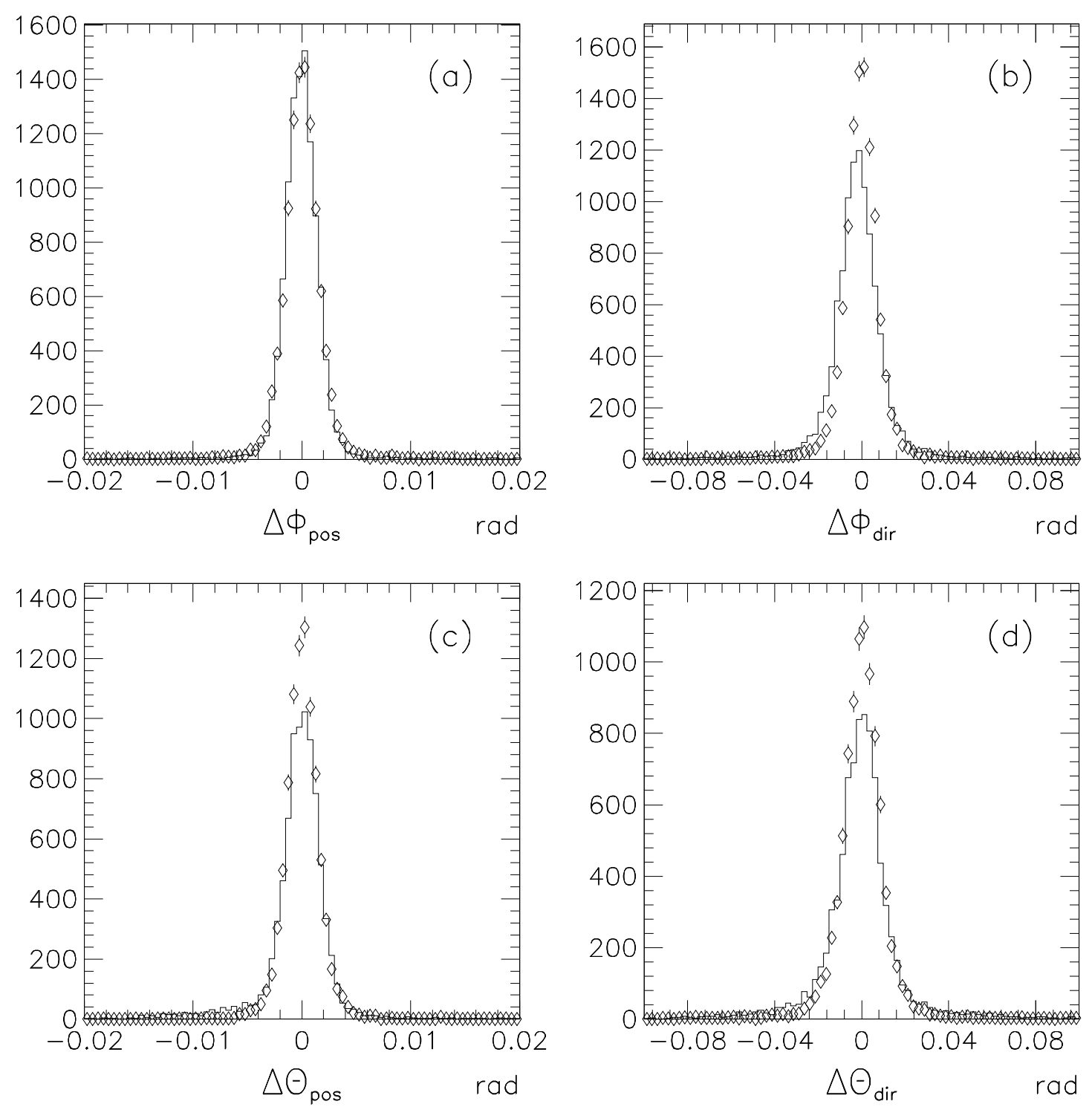

Figure 19: Matching of extrapolated tracks with MB muon segments for data (solid line) and Monte Carlo (points with errors) in muon pair events. The plots are normalized to equal numbers of events. The difference between the extrapolated central detector track and the track segment in the muon chambers is shown for:

(a) the position of the segment in $\phi$;

(b) the segment direction in $\phi$;

(c) the segment position in $\theta$;

(d) the segment direction in $\theta$. 


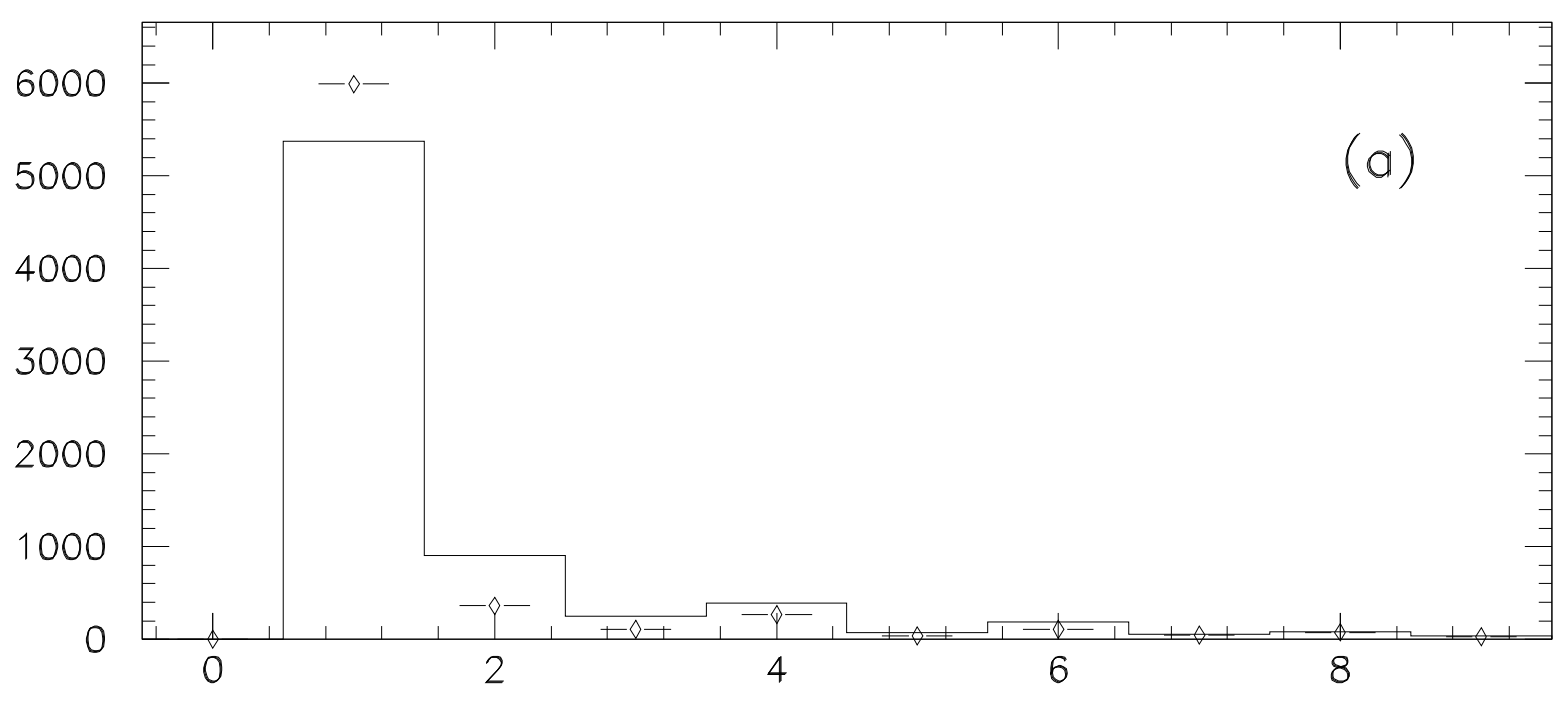

Number of muon segments

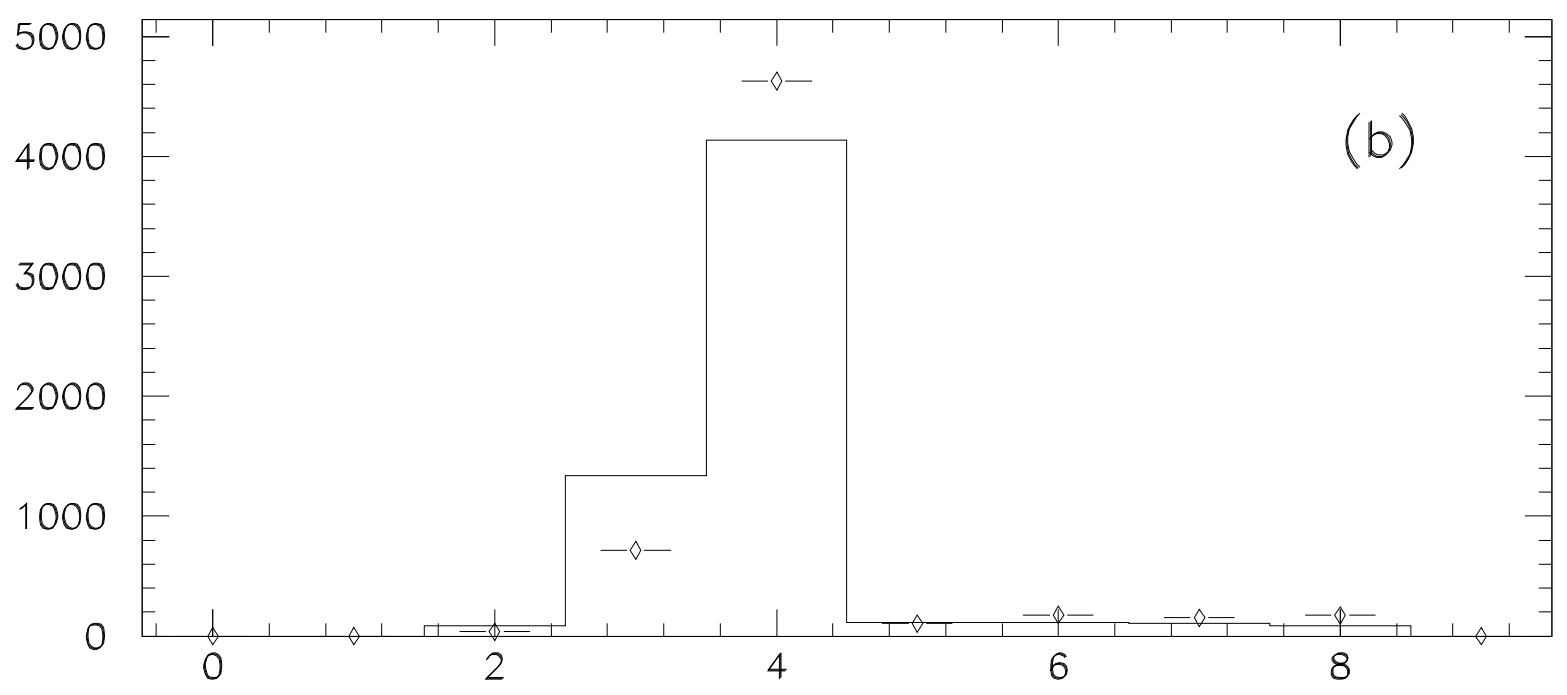

Number of $X$ hits per muon segment

Figure 20: Comparison between GOPAL (points with errors) and data (solid line) for the muon endcaps. The plots are normalized to equal numbers of events.

(a) The number of track segments reconstructed per muon in $\mu^{+} \mu^{-}$events;

(b) The number of hits per segment. 


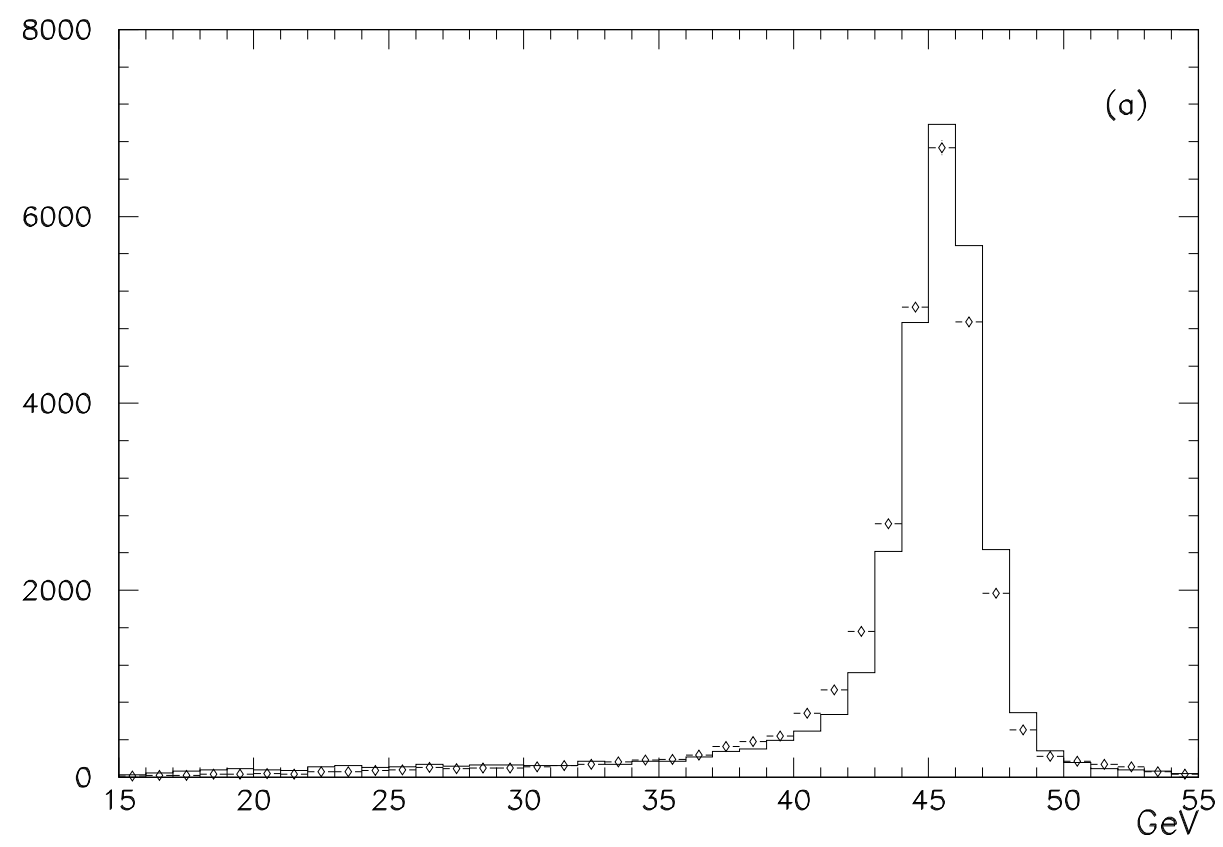

Energy, $\ominus>50 \mathrm{mrad}$
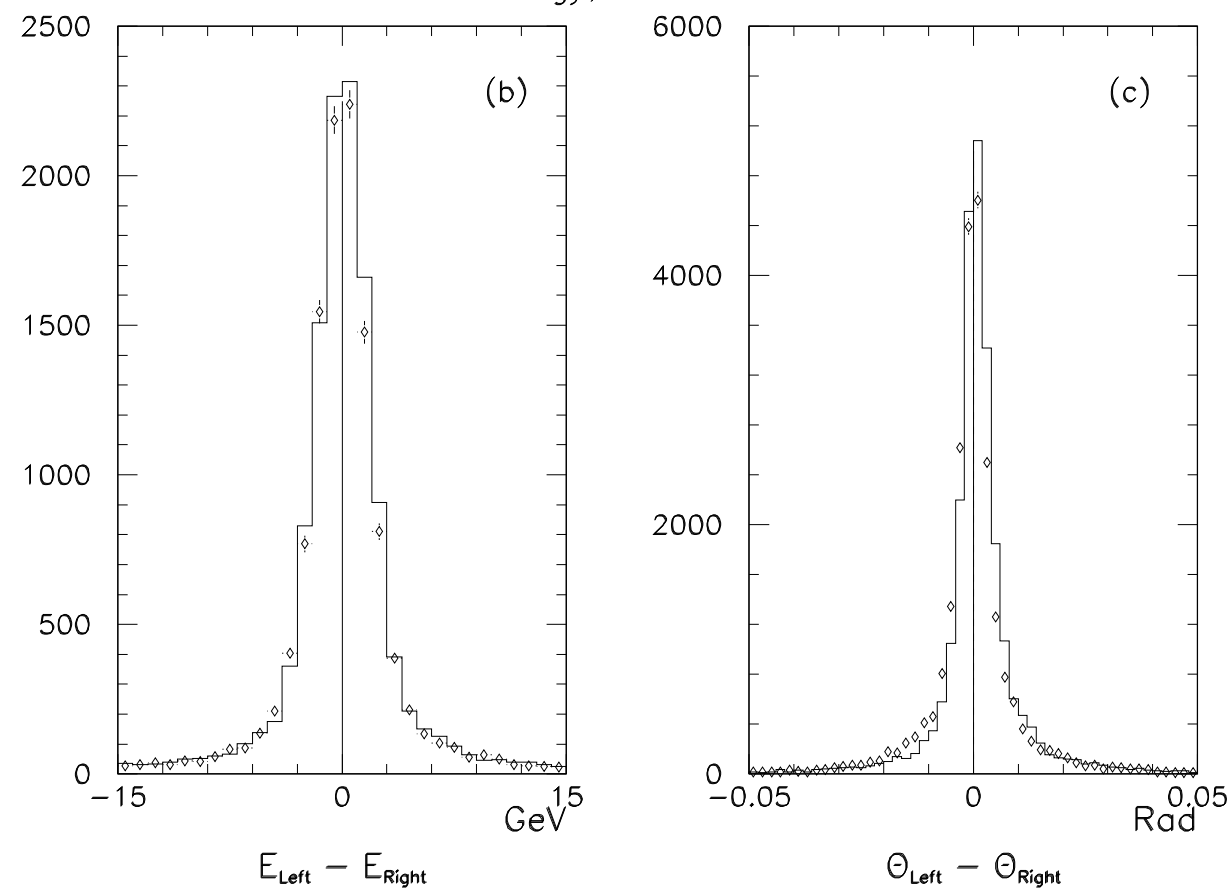

Figure 21: Comparison of data (solid line) and Monte Carlo (points with errors) for the Forward Calorimeter. The plots are normalized to equal numbers of events.

(a) Energy in a forward calorimeter for Bhabha scattering events with $\Theta>50$ mrad;

(b) Difference in Calorimeter energy between left and right end clusters;

(c) Difference in Calorimeter $\theta$ between left and right end clusters . 


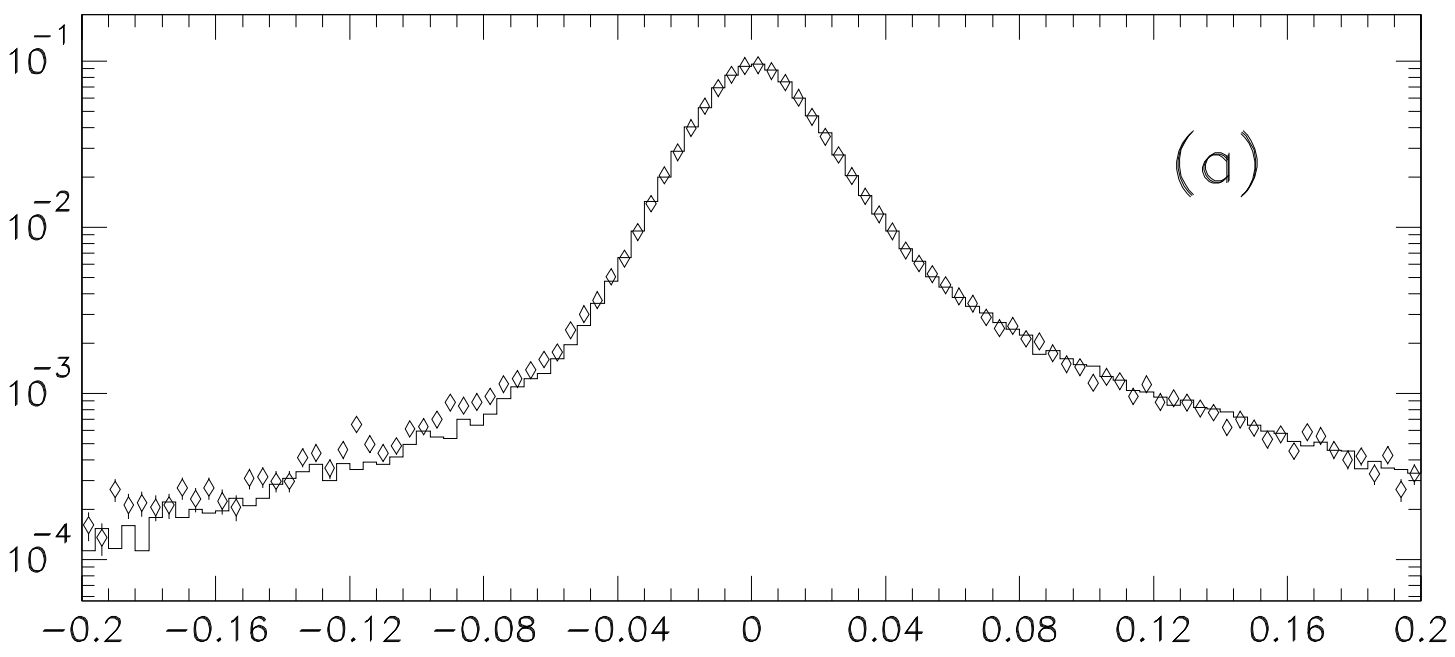

Signed Impact Parameter $(\mathrm{cm})$

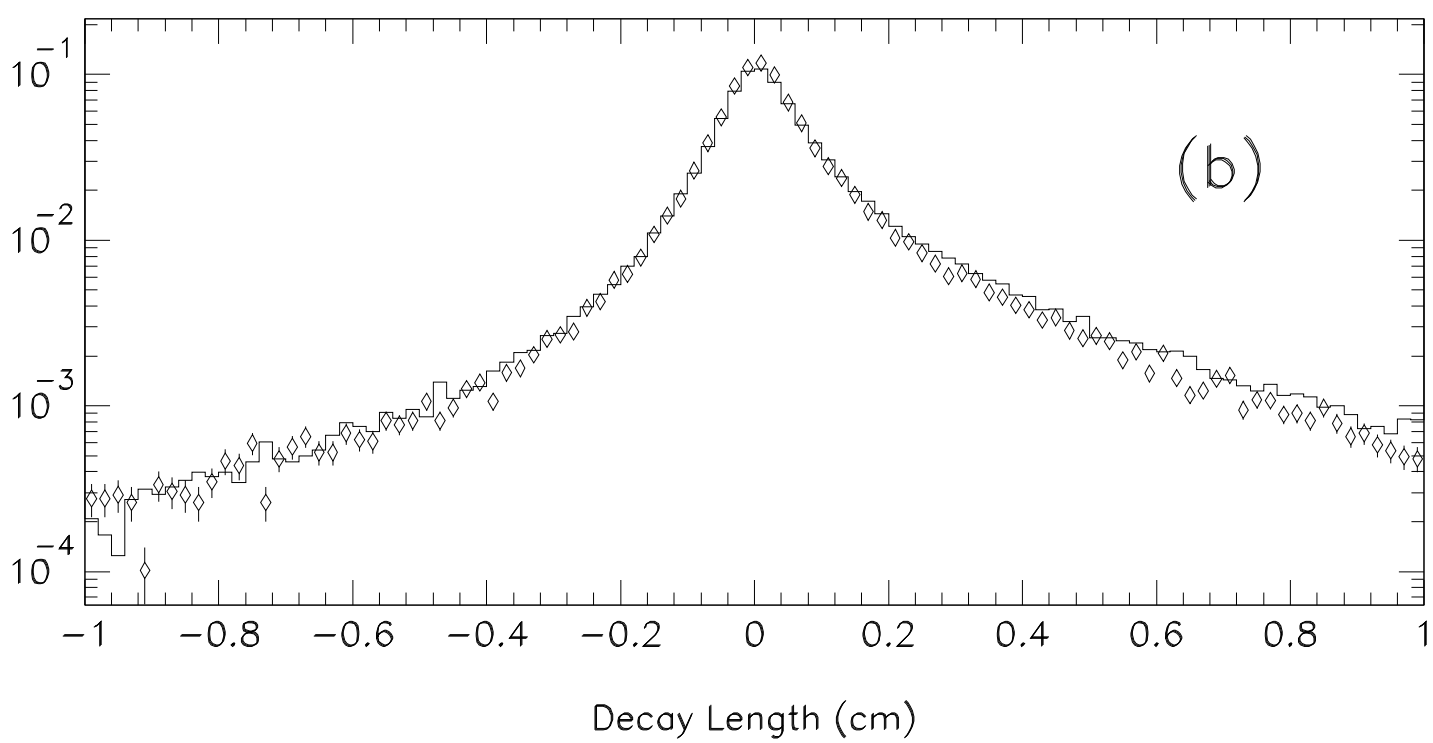

Figure 22: Distributions for the Central Detector simulation of hadronic events in "smear" mode, comparing data (solid line) with Monte Carlo (points with errors). The plots are normalized to equal numbers of events.

(a) The signed impact parameter to the main vertex in the $r-\phi$ projection (signed so that tracks crossing in front of the vertex are positive, and those crossing behind are negative);

(b) The decay length of jets (i.e. the distance of the reconstructed jet vertex from the main event vertex). In both cases an excess of positive values signifies the presence of hadrons containing heavy quarks decaying away from the main production point. 